\title{
Determination of saccharides and ethanol from biomass conversion using Raman spectroscopy: effects of pretreatment and enzyme composition
}

by

\section{Chien-Ju Shih}

A dissertation submitted to the graduate faculty

in partial fulfillment of the requirements for the degree of

\section{DOCTOR OF PHILOSOPHY}

\author{
Major: Analytical Chemistry \\ Program of Study Committee: \\ Emily A. Smith, Major Professor \\ Robert S. Houk \\ Patricia A. Thiel \\ L. Keith Woo \\ Andrew C. Hillier
}

Iowa State University

Ames, Iowa

2010

Copyright (C Chien-Ju Shih, 2010. All rights reserved. 
To My parents, Bob and Gerald 


\section{TABLE OF CONTENTS}

DEDICATION ii

TABLE OF CONTENTS iii

ACKNOWLEDGEMENTS $\quad$ V

CHAPTER 1: GENERAL INTRODUCTION 1

$\begin{array}{lr}\text { Dissertation overview } & 1\end{array}$

$\begin{array}{lr}\text { Research motivation and goals } & 2\end{array}$

Lignocellulosic biomass and pretreatment methods 3

$\begin{array}{lr}\text { Hydrolysis and fermentation } & 6\end{array}$

$\begin{array}{ll}\text { Raman spectroscopy } & 8\end{array}$

$\begin{array}{ll}\text { Figures } & 13\end{array}$

$\begin{array}{ll}\text { References } & 17\end{array}$

CHAPTER 2: DETERMINATION OF GLUCOSE AND ETHANOL AFTER ENZYMATIC HYDROLYSIS AND FERMENTATION OF BIOMASS USING RAMAN SPECTROSCOPY (A paper published in Analytica Chimica Acta (653) 2009, 200-206) 19

$\begin{array}{ll}\text { Abstract } & 19\end{array}$

$\begin{array}{ll}\text { Introduction } & 20\end{array}$

$\begin{array}{ll}\text { Materials and methods } & 24\end{array}$

$\begin{array}{ll}\text { Results and discussion } & 28\end{array}$

$\begin{array}{ll}\text { Conclusions } & 36\end{array}$

$\begin{array}{ll}\text { Acknowledgements } & 37\end{array}$

$\begin{array}{ll}\text { References } & 37\end{array}$

$\begin{array}{ll}\text { Tables } & 41\end{array}$

$\begin{array}{ll}\text { Figures } & 43\end{array}$

$\begin{array}{lr}\text { Supplemental materials and methods } & 49\end{array}$ 
CHAPTER 3: RAMAN SPECTROSCOPY MEASUREMENTS OF SACCHARIDES IN HYDROLYSATE USING DIFFERENT BIOMASS PRETREATMENT PROTOCOLS AND ENZYME COMPOSITIONS

Abstract

Introduction 58

Materials and methods

61

Results and discussion 65

Conclusions 72

Acknowledgements 72

References 72

Tables 75

Figures 79

CHAPTER 4: CONCLUSIONS

82

APPENDIX: DETECTION OF CADMIUM (II) IN WATER USING COLORIMETRICSOLID PHASE EXTRACTION (A paper published in SAE International, Technical

Paper 2005-01-28)

Abstract

Introduction

Experimental 86

Results and discussions 89

Conclusions 92

Acknowledgements 92

References 92

Table 95

Figures 96 


\section{ACKNOWLEDGEMENT}

What a long but amazing journey! I still remember when I made up my mind to major in Chemistry 20 years ago. I have been very lucky to have so many chances to explore the beauty of chemistry.

This thesis would have been impossible without my advisor Dr. Smith. Her patience, support and helpful guidance will always be remembered and appreciated. Thank you, Emily!

It is my pleasure to express my gratitude to several professors that helped me a lot including my committee members: Dr. Houk, Dr. Thiel, Dr. Woo and Dr. Hillier, thank you all for helping me not only on my research but also with my life in graduate school. I also want to thank Dr. Small and Dr. Lin for their encouragements, advices and sharing their enthusiasm about science with me. Many thanks to my first advisor Dr. Porter, who gave me a chance to participate in NASA project and taught me a valuable life lessonnobody can diminish you but yourself.

I can never express my appreciation enough to my parents Tzu-Ming Shih and Shu Chuan Yao who dedicate all of their time and resources educating me. There is no daughter luckier than me in this world. I also want to thank my sister Eva for always supporting me, especially in the down time. Facing similar challenges, while pursuing our doctorate degrees has brought us closer. Another very special family member to thank is my uncle who taught me everything about physics and was always very supportive at different stages of my life. I am sure he is proud of me and smiling in heaven. 
I am so grateful for all the love and support from my husband Bob in these 9 years. He makes my life full of laughter. My son Gerald has given me all the strength to finish this dissertation and his smile always lightens up my days. I am truly blessed to have wonderful in-laws, and I thank them for always understanding and offering help.

Last but not the least, I would like to thank my friends for all the support and always telling me I can do it! Ya-Ju, Cookie, Josephine, Cindy and Grace have offered their friendship for more than 20 years. Porter group members, especially my best friend April, Becky, Bob Lipert, Karen and Jill, thanks for helping me at difficult times. To Smith group members: it's been a great time to work with you guys and that makes it so difficult to say goodbye. Aunt Dodo, Cedric and Tommy, thank you all for your generous help, which has made my time in Ames very cheerful.

I believe that success belongs to those who are willing to be adventurous. I thank ISU and the Department of Chemistry for a good education. I hope I can keep an open and humble mind on this never-ending adventurous journey of science. Farewell! 


\section{CHAPTER 1: GENERAL INTRODUCTION}

\section{Dissertation overview}

This dissertation focuses on the development of facile and rapid quantitative Raman spectroscopy measurements for the determination of conversion products in producing bioethanol from corn stover. Raman spectroscopy was chosen to determine glucose, xylose and ethanol in complex hydrolysis and fermentation matrices. Chapter 1 describes the motives and main goals of this work, and includes an introduction to biomass, commonly used pretreatment methods, hydrolysis and fermentation reactions. The principles of Raman spectroscopy, its advantages and applications related to biomass analysis are also illustrated. Chapter 2 and 3 comprise two published or submitted manuscripts, and the thesis concludes with an appendix. In Chapter 2, a Raman spectroscopic protocol is described to study the efficiency of enzymatic hydrolysis of cellulose by measuring the main product in hydrolysate, glucose. Two commonly utilized pretreatment methods were investigated in order to understand their effect on glucose measurements by Raman spectroscopy. Second, a similar method was set up to determine the concentration of ethanol in fermentation broth. Both of these measurements are challenged by the presence of complex matrices. In Chapter 3, a quantitative comparison of pretreatment protocols and the effect of enzyme composition are studied using systematic methods. A multipeak fitting algorithm was developed to analyze spectra of hydrolysate containing two analytes: glucose and xylose. Chapter 4 concludes with

a future perspective of this research area. An appendix describes a convenient, rapid spectrophotometric method developed to measure cadmium in water. This method requires relatively low cost instrumentation and can be used in microgravity, such as space shuttles or 
the International Space Station. This work was performed under the supervision of Professor Marc Porter while at Iowa State University.

\section{Research motivation and goals}

Research related to producing biofuel from bio-renewable resources, especially bioethanol from biomass, has grown significantly in the past decade due to the high demand and rising costs of fossil fuels [1]. More than 3 percent of the energy consumption in the U.S. is derived from renewable biomass, mostly through industrial heat and steam production by the pulp and paper industry, and electricity generation from municipal solid waste (MSW) and forest industry residues [2]. The utilization of food-based biomass to make fuels has been widely criticized because it may increase food shortages throughout the world and raise the cost of food. Thus, nonfood-based and plentiful lignocellulosic feedstocks, such as corn stover, perennial grass, bagasse, sorghum, wheat/rice straw, herbaceous and woody crops, have great potential to be new bio-renewable sources for energy production $[3,4]$. Given that many varieties of biomass are available, there is need for a rapid, simple, high-throughput method to screen the conversion of many plant varieties. The most suitable species for each geographic region must be determined, as well as the optimal stage of harvest, impacts of environmental conditions (temperature, soil, $\mathrm{pH}$, etc.). Various genetically modified plants should be studied in order to establish the desired biomass in bioethanol production. The main screening challenge, however, is the complexity of plant cell wall structures that make reliable and sensitive analysis difficult. 
To date, one of the most popular methods to produce lignocellulosic ethanol is to perform enzymatic hydrolysis followed by fermentation of the hydrolysate with yeast. There are several vital needs related to the field of chemistry that have been suggested as primary research foci needed to effectively improve lignocellulosic ethanol production. These topics include overcoming the recalcitrance of cellulosic biomass, the pervasiveness of pretreatment, advanced biological processing and better feedstocks [3]. In this thesis, a novel approach using Raman spectroscopy has been developed to address important issues related to bioethanol generation, which will aid the research aimed to solve the topics mentioned above.

\section{Lignocellulosic biomass and pretreatment methods}

The plant cell wall consists of cellulose (40-60\% dry mass), hemicellulose (20-40\% dry mass), polyphenolic lignin (10-25\% dry mass) and other extractable components [4, 5]. Cellulose is present as highly-ordered microfibrils, each containing up to 36 glucan chains, equivalent to thousands of glucose units [6]. The microfibrils of cellulose tightly associate with hemicellulose, a polymer with various monosaccharide units. The hemicellulose also covalently bonds with lignin, a polymeric material composed of phenylpropanoid units derived from three hydroxycinnamyl alcohol derivatives : p-coumaryl, coniferyl, and sinapylalcohols [7]. Lignin provides mechanical strength to the plant tissue and is the primary barrier to cellulose accessibility to enzymes.

The association between cellulose and the other two cell wall components, as well as the crystallinity of cellulose are two key properties that must be disrupted when trying to 
obtain high efficiencies of cellulose breakdown to glucose. This in turn affects the conversion of glucose to ethanol. Therefore, a pretreatment step is prerequisite for efficiently hydrolyzing cellulose or hemicellulose in the biomass. The purpose of pretreatment is to disrupt the highly-coordinated cellulose, hemicellulose and lignin interactions in the cell wall, and make the polysaccharide chains more accessible to the enzymes. A schematic of the effects of biomass pretreatment on the cell wall polymer interactions is shown in Figure 1 [8]. Ideally, pretreatment methods should meet following requirements: low cost, ability to reduce or remove the maximum amount of extractives and inhibitors including lignin, retain a majority of the polysaccharides, consume little energy, consume few chemicals and produce no pollution.

There have been numerous pretreatment methods developed; in general, they can be classified to four groups: physical (milling, pyrolysis, $\gamma$-irradiation, and microwave irradiation), chemical (alkali, acid, gases, oxidizing agents, solvent extraction and cellulose dissolving agents), physiochemical (explosion using $\mathrm{CO}_{2}, \mathrm{SO}_{2}$, steam, or ammonia) and biological (fungi) [9]. Each pretreatment method has its strength and weakness and scientists have compared several leading pretreatment protocols on biomass from different aspects [8, 10]. In this chapter, the discussion will focus on two most common chemical pretreatment methods-dilute acid and ammonia pretreatment.

Among all the pretreatment methods, the most widely used and studied chemical pretreatment method utilized to date is dilute-acid pretreatment. Although this is usually performed using sulfuric acid [11, 12], peracetic acid has also been used [13]. The acid concentration used in dilute acid pretreatment is in the range of 0.5 to $3.0 \mathrm{wt} \%$, and it is 
suitable for industrial application. It has been proven to be very effective in disrupting the hemicellulose interaction with lignocellulose. The removal of hemicellulose increases the accessibility of the enzyme to the cellulose, subsequently increasing the efficiency of enzymatic hydrolysis. Furthermore, it could yield close to the theoretical yield for carbohydrates when combined with the hydrolysis of hemicellulose (xylose, $70 \%$ to $90 \%$ yield) [14]. Although combined sugar yield is high using acid for biomass pretreatment, there are several drawbacks. First, the necessity of neutralization before fermentation generates gypsum; second, this pretreatment process involves high capital investment, large chemical consumption and acid recovery costs [9]. Most important of all, the relatively large amount of extractives and lignin left in the solid portion of the pretreated biomass and the byproducts from this process consequently impede fermentation reaction. A systematic study using various compositions of these degradation by-products has proven that treatment at lower $\mathrm{pH}$ results in more fermentation inhibitors [15].

Another chemical found to be beneficial for disruption of crystalline structure of the cell wall is ammonia, which can cleave C-O-C bonds in lignin and glucoronic acid ester crosslinks [16]. Several pretreatment methods have been developed using ammonia, such as ammonia recycled percolation (ARP) [16], ammonia fiber explosion (AFEX) [17, 18], and soaking in aqueous-ammonia (SAA) at moderate or room temperature [19, 20]. Among them, SAA provides advantages of low process energy, easy set-up and removal of 62 to $85 \%$ lignin while retaining almost 90 to $100 \%$ glucan and 40 to $85 \%$ xylan. As previously mentioned, removing hemicellulose can enhance the hydrolysis efficiency due to the increase in cellulose porosity. Detoxification is needed after acid pretreatment before biomass can be 
converted. The detoxification step is not very well-established and it is costly in bioethanol production process. The cellulose in ammonia-pretreated corn stover, has high digestibility by cellulase while significant amounts of hemicellulose remain in the solid portion. The coexistence of hemicellulose and cellulose in the pretreated biomass makes it possible to hydrolyze both polysaccharides with cellulase (or with the addition of hemicellulase), simultaneously. The hydrolysate also has few inhibitors, thus a detoxification step is not necessary.

Significant effort has been put into understanding how hydrolysis or fermentation efficiencies relate to the structure of the cell wall, and the correlation and location of each cell wall component $[21,22]$. These studies provided valuable information to improve the bioethanol process by finding the biomass with optimal conversion efficiencies. However, because of the complexity and heterogeneous nature of the biomass, the true hydrolysis and fermentation efficiency after pretreatment must be experimentally determined for each biomass sample. Facile analysis methods have to be established in order to fairly compare each biomass and each pretreatment method.

\section{Hydrolysis and fermentation}

Hydrolysis of cellulose and hemicellulose can be carried on with dilute acid, concentrated acid, or specific enzymes. Typical hydrolysis products of these polysaccharides are a variety of oligo- or mono-saccharides. Dilute acid hydrolysis is the oldest technology

for converting biomass to ethanol, and is usually a two-stage process [23, 24]. Milder 
reaction conditions are used for the first stage to pretreat biomass and hydrolyze hemicellulose, mainly 5-C sugar (xylose, arabionose). In the second stage, harsher conditions are used to hydrolyze cellulose to 6-C sugar (glucose). If the first stage is skipped, the 5-C sugars that would have been produced in the first stage of reaction will be converted to other chemicals, such as furfural [5]. The advantages of using concentrated acid includes cut down the time required for hydrolysis, the cost of chemicals, neutralization and equipment maintenance.

Enzymatic hydrolysis has been considered key to cost-effective bioethanol in the long run, and the reaction is carried out with mainly cellulase and hemicellulase for cellulose and hemicellulose, respectively. The advantages of using enzyme (cellulase) over acid is to eliminate corrosion problems and lower maintenance costs with mild processing conditions to give high yields [5]. The cellulases break down $\beta$-1,4-glucan or $\beta$-D-glucosidic linkages of cellulose and generate sugars such as cellooligosaccarides, cellobiose and glucose. Cellulases and hemicellulases are mixtures of enzymes usually produced from fungi (Trichoderma reesei, Trichoderma viride, Aspergillus niger, etc.) [25]. Traditionally these enzymes are divided into three categories: endo-1,4- $\beta$-glucanases (EC 3.2.1.4, EGs), exoglucanases, or cellobiohydrolases (EC 3.2.1.91, CBHs), and $\beta$-glucosidases (EC 3.2.1.21, BGLs). These enzymes are believed to work synergistically: CBHs target cellulose chains from both ends, whereas the EGs target random locations away from polysaccharide chain ends; both enzymes degrade cellulose to cellobiose dimers or oligomers which are then converted to glucose by BGLs [26]. A schematic showing the main hydrolysis products including sugars and inhibitors is shown in Figure 2 [27]. Numerous factors have been reported to have 
impacts on hydrolysis efficiency, such as properties of pretreated substrates (crystallinity, level of deacetylation and delignification [28]), cellulase activity and composition, and reaction conditions (buffer $\mathrm{pH}$, temperatures, etc.).

Monosaccharides obtained from hydrolysis reactions can be converted to ethanol via fermentation with yeast, for example, Saccharomyces cerevisiae. Separate hydrolysis and fermentation (SHF) reactions, or simultaneous saccharifications and fermentation (SSF) reactions can be performed [29]. Typical fermentation efficiencies are in the range of 76 83\% [30]. SHF is the traditional method to produce ethanol and the advantage of this process is that each step can be performed in optimized condition (buffer $\mathrm{pH}$ and temp) and detoxification steps can be added in between to remove inhibitors, if necessary. To perform a successful SSF, the compatibility of reaction conditions (reagents, enzyme and yeast concentration, temperature, etc.) is a key factor. Hydrolysis conditions have to be modified to incorporate fermentation conditions and minimize the amount of inhibitors being produced. SSF has advantages including: higher product yield, higher cellulase hydrolysis rates, lower enzyme loading, and the activity of the yeast can also be improved if the ethanol can be recovered continuously [31]. It is worthy to note that in order to maximize the total sugar yield, development of yeast that could ferment carbohydrates from hemicellulose is critical $[32,33]$.

\section{Raman spectroscopy}

The phenomenon of inelastic scattering of light was first observed experimentally by a physics professor C. V. Raman in 1928 [34].This characteristic property of light was then 
named in his honor. Monochromatic incident light, nowadays from a laser, interacts with the molecules and a small portion of the photons are scattered with a frequency shift relative to the incident beam corresponding to a vibrational energy level as shown in Figure 3. The Rayleigh scatter, an elastic process, is the most intense frequency among the three shown in Figure 3 because a majority of the scattered photons do not change energy. There are two types of Raman scatter- Stokes and anti-Stokes. Stokes scatter has smaller frequencies than the incident light and anti-stokes lines have higher energy than the incident light. Stokes lines are usually more intense than anti-Stokes lines because the population of the lower vibration state is larger at room temperature. Anti-stokes lines becomes even weaker at higher frequency due to decreased population of the excited vibrational states [35]. In most cases, the Raman scatter collected and analyzed are the Stokes lines. When there is fluorescence interference from the sample, the anti-Stokes spectrum is preferred for analysis. The frequency shift is a measure of the energy of the vibration, and Raman spectroscopy is a tool that can provide valuable information for molecular characterization of complex systems [36].

The selection rule required for Raman scattering to occur is a change in polarizibility during the vibration [37], which is different from another vibrational spectroscopic technique- infrared spectrometry (IR). In IR spectrometry, the frequency of the incident light has to match the energy difference between ground and excited vibrational state; therefore, the energy loss of the incident light can be detected. The vibration in IR spectrometry can only be observed when there is a change in dipole moment during vibration.

Raman spectroscopy has several advantages in the application related to bioethanol conversion processes. Raman spectroscopy is a non-destructive method and only requires 
small sample volumes. In addition, the analysis time is relatively short. Most important of all, Raman spectroscopy, unlike IR spectrometry, doesn't exhibit interference from water. Raman spectroscopy is suitable for analyzing aqueous samples without the use of attenuated total reflection methods [38].

There are several important factors that impact the performance of Raman spectroscopy when measuring biomass samples. First, high fluorescence background values are often observed using visible incident wavelengths in Raman spectroscopy; thus, near IR incident lasers have become a popular choice for Raman spectroscopy measurements of biological samples, including plant materials. Near IR sources emit low incident energy photons that can minimize both sample damage and fluorescence background, which is problematic for most biological samples. Second, combining the use of a sensitive CCD camera, it is possible to obtain dispersive Raman spectra of most analytes with high sensitivity.

The instrument used in this work is shown in Figure 4. The main components in this set up include: a $785 \mathrm{~nm}$ near-IR (NIR) laser, 40x magnification objectives, a spectrometer and a NIR-enhanced CCD. The spectral axis (pixel to wavenumber) was calibrated using peaks in a spectrum of 50:50 (v:v) acetonitrile:toluene solutions. A home-built sample holder was used for liquid measurement. Approximately $1.5 \mathrm{~mL}$ of the sample solution was loaded into a sample holder, which was fabricated with a glass coverslip bottom and Teflon walls.

Standard spectra of analytes (glucose, xylose, ethanol in this work) with different concentrations can be generated to confirm characteristic Raman shifts and peak intensities or areas for quantitative work. Peak area is generally preferred because the noise can be averaged throughout the width of the peak; thus, the noise has less impact on the data. The 
peak height can also be used to accurately quantify analyte concentration [39] once it is confirmed to be proportional to analyte concentration. For the analytes used in this work, both peak area and peak height have been examined during method development, and the results were in close agreement. Peak intensity was chosen for all the work reported in this dissertation.

Hydrolysis and fermentation broths contain numerous components that can contribute to the Raman signal, so it is important to distinguish analyte peaks from matrix peaks. Usually, if available, a blank will be prepared with the same matrix as the sample minus analyte. This blank spectrum can be subtracted from the sample spectrum to generate a spectrum representative of the analyte. If a blank is not available, usually when the matrix is very complicated, standard addition can be used to determine analyte concentrations. Sometimes an analyte standard spectrum can’t be generated. In this case, spectral subtraction can yield useful information. For example, a spectrum of native wood lignin can be generated by subtracting the native wood spectrum with the spectrum of completely delignified wood [40].

Multipeak fitting algorithms can be used to determine concentrations when no features in the overlap spectrum are distinct enough for single analyte. First, single-analyte spectra at different concentrations are acquired, and then bands in chosen region are fitted with multiple peaks. Appropriate peaks are chosen for each analyte and to generate calibration curves. There are several parameters can be used to build a quantitation model including: position, width, and intensity ratio of the peaks. In our work, positions and widths obtained from single-analyte spectra are fixed (held) when we deconvolute mixture spectra. 
Intense peaks that could represent each analyte are chosen, and the concentration of each analyte can be calculated using the intensities of chosen peaks and the calibration curves built earlier. This method allowed multiple analytes with overlap spectra to be analyzed simultaneously. 


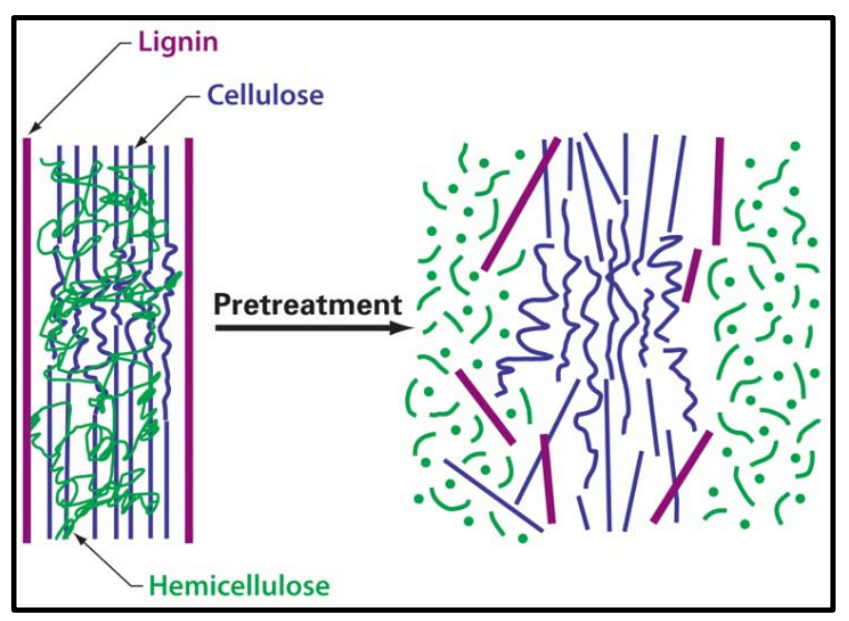

Figure 1 Schematic of goals of pretreatment on lignocellulosic material 


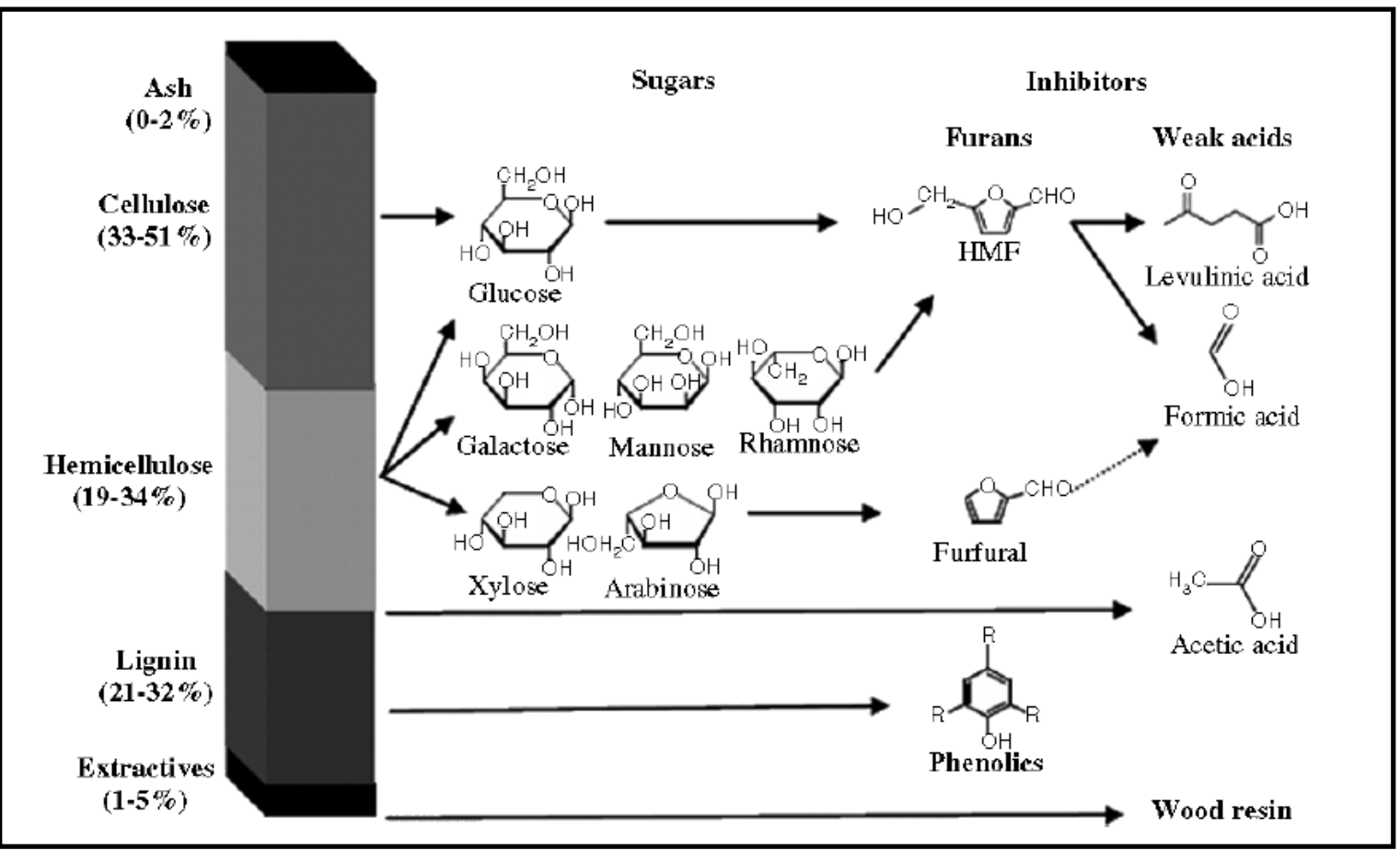

Figure 2 Average composition of lingocellulosic biomass and main derived hydrolysis products 


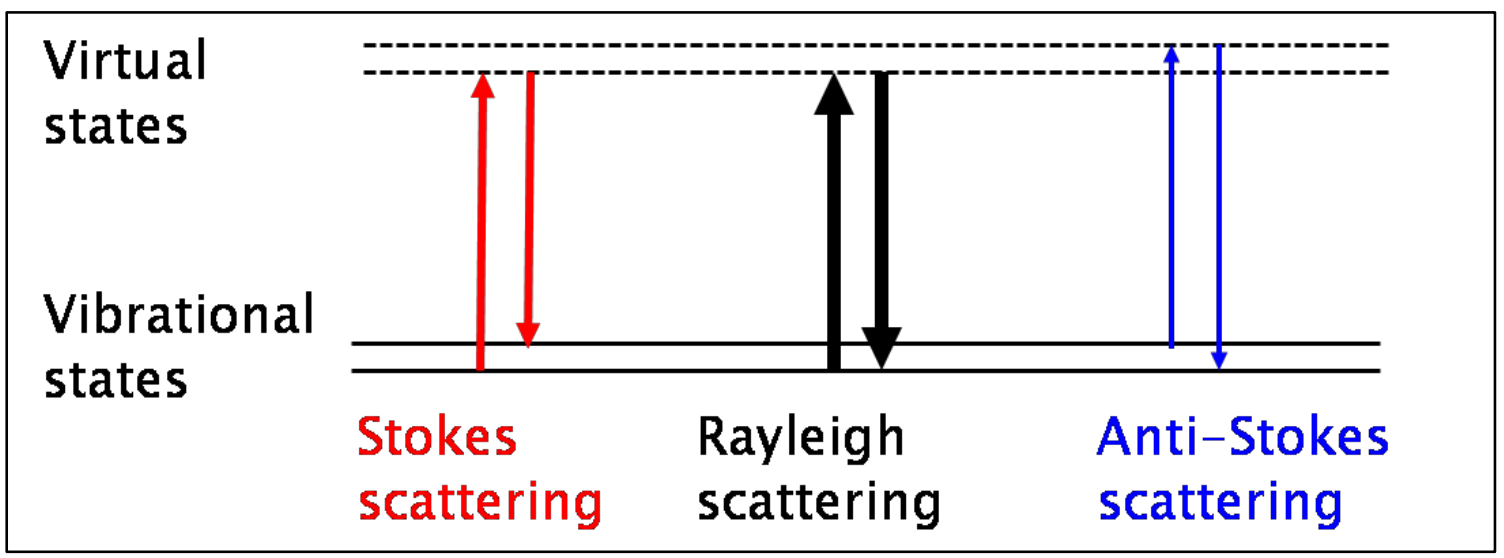

Fig 3 Diagram of Rayleigh and Raman scattering processes. 


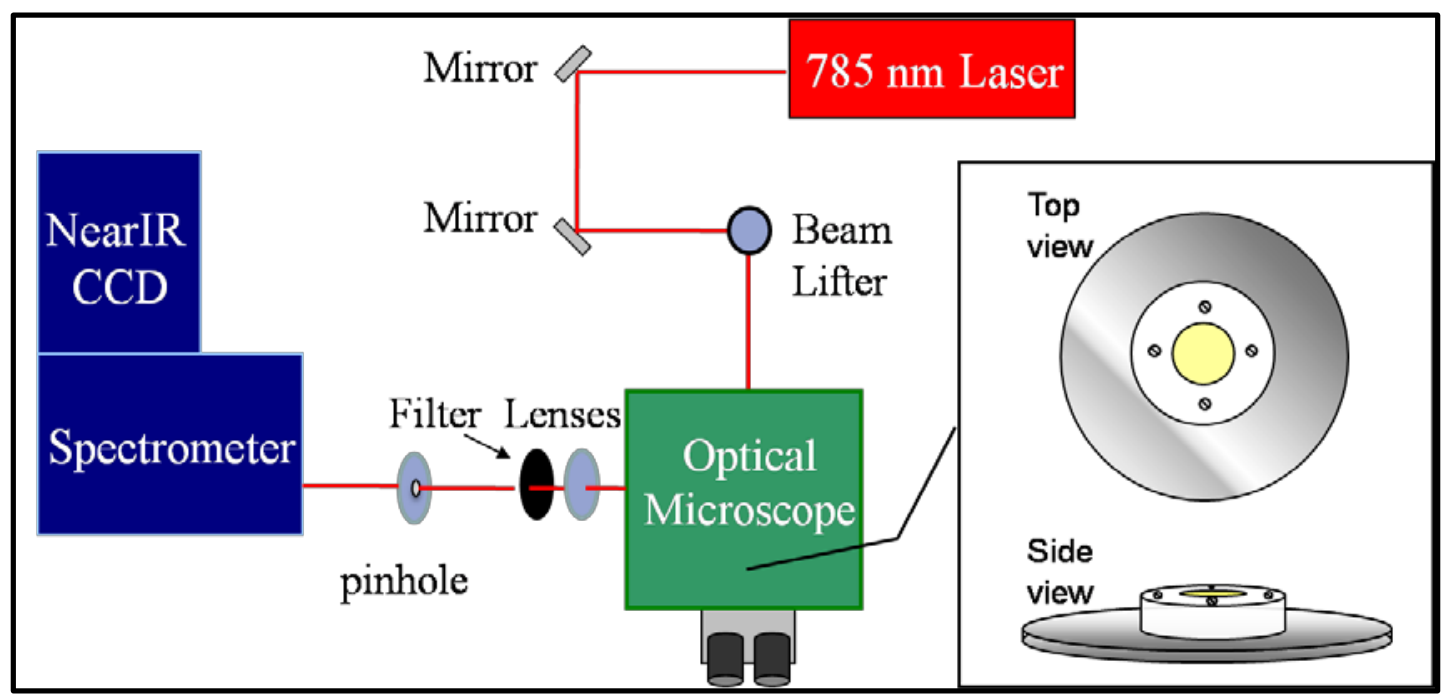

Fig 4 Diagrams of a home-built Raman microspectroscopy instrument configuration and sample holder used in all Raman spectroscopic experiments described in this thesis. 


\section{Reference}

[1] J.B. Kristensen, L.G. Thygesen, C. Felby, H. Jorgensen, T. Elder, Biotechnology for biofuels, 1 (2008) 5.

[2] U.S. DOE, http://www.eia.doe.gov/cneaf/solar.renewables/page/trends/table1.html, 2008.

[3] C.E. Wyman, Trends in Biotechnology, 25 (2007) 153.

[4] M. Knauf, M. Moniruzzaman, Int. Sugar J., 106 (2004) 147.

[5] C.N. Hamelinck, G. Van Hooijdonk, A.P.C. Faaij, Biomass and Bioenergy, 28 (2005) 384.

[6] C. Somerville, S. Bauer, G. Brininstool, M. Facette, T. Hamann, J. Milne, E. Osborne, A. Paredez, S. Persson, T. Raab, S. Vorwerk, H. Youngs, Science (Washington, DC, United States), 306 (2004) 2206.

[7] R. Hatfield, R.S. Fukushima, Crop Science, 45 (2005) 832.

[8] N. Mosier, C. Wyman, B. Dale, R. Elander, Y.Y. Lee, M. Holtzapple, M. Ladisch, Bioresource Technology, 96 (2005) 673.

[9] R.S. Laxman, A.H. Lachke, Handbook of Plant-Based Biofuels, 2009, p. 121.

[10] R. Kumar, C.E. Wyman, Bioresource Technology, 100 (2009) 4203.

[11] A. Mohagheghi, D.J. Schell, Biotechnology and Bioengineering, 105 (2009) 992.

[12] C.I. Ishizawa, T. Jeoh, W.S. Adney, M.E. Himmel, D.K. Johnson, M.F. Davis, Cellulose

(Dordrecht, Netherlands), 16 (2009) 677.

[13] L.C. Teixeira, J.C. Linden, H.A. Schroeder, Appl Biochem Biotechnol, 84-86 (2000) 111.

[14] C. Liu, C.E. Wyman, Industrial \& Engineering Chemistry Research, 43 (2004) 2781.

[15] P. Persson, J. Andersson, L. Gorton, S. Larsson, N.-O. Nilvebrant, L.J. Jönsson, J. Agric.

Food Chem., 50 (2002) 5318.

[16] T.H. Kim, J.S. Kim, C. Sunwoo, Y.Y. Lee, Bioresource Technology, 90 (2003) 39.

[17] V. Balan, B. Bals, S.P.S. Chundawat, D. Marshall, B.E. Dale, Methods in Molecular Biology (Totowa, NJ, United States), 581 (2009) 61.

[18] F. Teymouri, L. Laureano-Perez, H. Alizadeh, B.E. Dale, Bioresource Technology, 96 (2005) 2014.

[19] M. Galbe, G. Zacchi, Advances in Biochemical Engineering/Biotechnology, 108 (2007) 41.

[20] T.H. Kim, Y.Y. Lee, Applied Biochemistry and Biotechnology, 137-140 (2007) 81.

[21] N. Gierlinger, M. Schwanninger, Plant Physiology, 140 (2006) 1246.

[22] D.E. Akin, W.H. Morrison, III, L.L. Rigsby, F.E. Barton, II, D.S. Himmelsbach, K.B. Hicks, Applied Biochemistry and Biotechnology, 129-132 (2006) 104.

[23] M.J. Beck, R.C. Strickland, Biomass, 6 (1984) 101.

[24] K.H. Kim, M.P. Tucker, Q.A. Nguyen, Biotechnol. Prog., 18 (2002) 489.

[25] J.P.H. Van Wyk, M.A. Mogale, T.A. Seseng, Biotechnology Letters, 22 (2000) 491.

[26] S.N. Freer, Journal of Biological Chemistry, 268 (1993) 9337.

[27] J.R. Almeida, T. Modig, A. Petersson, B. Hähn-Hägerdal, G. Lidén, M.F. Gorwa-Grauslund, Journal of Chemical Technology \& Biotechnology, 82 (2007) 340.

[28] S. Kim, M.T. Holtzapple, Bioresource Technology, 97 (2005) 583.

[29] L.C. Teixeira, J.C. Linden, H.A. Schroeder, Biomass: A Growth Opportunity in Green

Energy and Value-Added Products, Proceedings of the Biomass Conference of the Americas, 4th, Oakland, Calif., Aug. 29-Sept. 2, 1999, 1999, p. 887.

[30] K. Öhgren, J. Vehmaanperä, M. Siika-Aho, M. Galbe, L. Viikari, G. Zacchi, Enzyme Microb. Technol., 40 (2007) 607.

[31] R. Anish, M. Rao, Handbook of Plant-Based Biofuels, 2009, p. 159.

[32] H. Punnapayak, G.H. Emert, Biotechnology Letters, 8 (1986) 63.

[33] I. Romeroa, S. Sáncheza, M. Moyaa, E. Castroa, E. Ruiza, V. Bravob, Biochemical Engineering Journal, 36 (2007) 108. 
[34] C.V. Raman, K.S. Krishnan, Nature (London, United Kingdom), 121 (1928) 501.

[35] E. Smith, G. Dent, Modern Raman spectroscopy: a pratical approach, Joh Wiley \& Sons, Ltd, West Sussex, England, 2005.

[36] R. Bhargava, M.D. Schaeberle, I.W. Levin, in: V.G. Gregoriou, M.S. Braiman (Eds.), Vibrational spectroscopy of Biological and Polymeric Materials, CRC Press, Boca Raton, FL, 2006, p. 430.

[37] J.D.J. Ingle, S.R. Crouch, Spectrochemical analysis, Prentice-Hall Inc., Upper Saddle River, 1988.

[38] J. De Gelder, K. De Gussem, P. Vandenabeele, L. Moens, Journal of Raman Spectroscopy, 38 (2007) 1133.

[39] S.Y. Lin, C.W. Dence, Editors, Methods in Lignin Chemistry, 1994.

[40] S.M. Ehrhardt, 1984, p. 341 pp. 


\title{
CHAPTER 2. DETERMINATION OF GLUCOSE AND ETHANOL AFTER ENZYMATIC HYDROLYSIS AND FERMENTATION OF BIOMASS USING RAMAN SPECTROSCOPY
}

A paper published in Analytica Chimica Acta (653) 2009, 200-206*

Chien-Ju Shih and Emily A. Smith

\begin{abstract}
Raman spectroscopy has been used for the quantitative determination of the conversion efficiency at each step in the production of ethanol from biomass. The method requires little sample preparation; therefore, it is suitable for screening large numbers of biomass samples and reaction conditions in a complex sample matrix. Dilute acid or ammonia-pretreated corn stover was used as a model biomass for these studies. Ammonia pretreatment was suitable for subsequent measurements with Raman spectroscopy, but dilute acid-pretreated corn stover generated a large background signal that surpassed the Raman signal. The background signal is attributed to lignin, which remains in the plant tissue after dilute acid pretreatment. A commercial enzyme mixture was used for the enzymatic hydrolysis of corn stover, and glucose levels were measured with a dispersive 785 nm Raman
\end{abstract}

\footnotetext{
* Reprint with permission from Analytica Chimica Acta

Copyright @ 2009 ELSEVIER
} 
spectrometer. The glucose detection limit in hydrolysis liquor by Raman spectroscopy was 8

$\mathrm{g} \mathrm{L}^{-1}$. The mean hydrolysis efficiency for three replicate measurements obtained with Raman spectroscopy ( $86 \pm 4 \%)$ was compared to the result obtained using an enzymatic reaction with

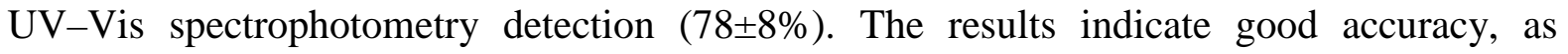
determined using a Student's t-test, and better precision for the Raman spectroscopy measurement relative to the enzymatic detection assay. The detection of glucose in hydrolysis broth by Raman spectroscopy showed no spectral interference, provided the sample was filtered to remove insoluble cellulose prior to analysis. The hydrolysate was further subjected to fermentation to yield ethanol. The detection limit for ethanol in fermentation broth by Raman spectroscopy was found to be $6 \mathrm{~g} \mathrm{~L}^{-1}$. Comparison of the

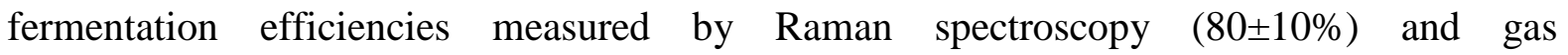
chromatrography-mass spectrometry $(87 \pm 9 \%)$ were statistically the same. The work demonstrates the utility of Raman spectroscopy for screening the entire conversion process to generate lignocellulosic ethanol.

\section{Introduction}

More than 3\% percent of U.S. energy consumption is derived from renewable biomass [1], predominantly in the form of fermented simple sugars derived from grains or sugar cane. In order to achieve the goal of increasing biorenewable fuel production, and minimize the concerns over grain-based ethanol, the more abundant cellulosic feedstocks such as corn stover, perennial grass, bagasse, wheat/rice straw and forestry/industry residue [2] must be used for commercial ethanol production. In these feedstocks, it is the polymers of 
the plant cell walls that contain the raw materials for ethanol production. Plant cell walls consist mostly of lignin tightly associated with hemicellulose and cellulose [3].

In contrast to the fermentation of simple sugars, a multi-step reaction is employed to hydrolyze cellulose to glucose, and subsequently ferment this to ethanol (Fig. 1). Lignin in the cell wall interferes with the enzymatic hydrolysis of the sugar polymers and a critical pretreatment step is required to disrupt the close association of the cell wall constituents [4]. Many pretreatment protocols are found in the literature, and each reports advantages and inferred disadvantages relative to other pretreatment steps. Dilute acid pretreatment breaks down the cell wall structure and exposes the cellulose for increased enzyme accessibility [5]. Approximately $70 \%$ of the lignin, $5 \%$ of the hemicellulose, and $95 \%$ of the cellulose remains after dilute acid pretreatment [6]. Ammonia pretreatment not only breaks down the cell wall constituents, but also removes a portion of the lignin from the cell wall [7]. Approximately $15 \%$ of the lignin, $40 \%$ of the hemicellulose, and $90 \%$ of the cellulose remains after ammonia pretreatment of corn stover [6]. After hydrolysis of the cellulose primarily through enzymatic routes, the simple sugars are fermented to ethanol. The composition of the biomass will vary depending on the growing locale, time of year, growth conditions, etc. [8]. The methods used to efficiently convert different feedstocks to ethanol will also vary with the composition. Much effort is focused on connecting efficient conversion methods with specific plant materials to produce the highest ethanol yields.

Facile, rapid methods for measuring all steps in the conversion of biomass to ethanol must accompany the research efforts aimed at maximizing conversion efficiencies. Ideally, these screening methods should be suited for varied biomass compositions, be capable of identifying analytes in complex hydrolysis and fermentation matrices, require little sample 
preparation and provide rapid analyses. To date, common methods for determining glucose in hydrolysate include high performance liquid chromatography (HPLC) [8-12], electrochemical methods [13] and ultraviolet (UV)-visible absorption spectrophotometry [14]. HPLC with refractive index detection or low-temperature evaporative light-scattering detection has provided efficient separation and quantification of sugars. However, chromatography methods have been inherently low-throughput techniques, requiring sample preparation and potentially long analysis times. UV-vis and electrochemical methods primarily use enzymes either in solution or immobilized on electrodes that specifically react with glucose. An example is the glucose HK assay, which utilizes a two-step reaction with hexokinase and glucose-6-phosphate dehydrogenase to generate an absorbing species, NADH. The absorbance at $340 \mathrm{~nm}$ is directly proportional to the glucose concentration in the solution [15]. Most detection schemes that rely on enzymatic reactions require an incubation step, and results may vary if uniform incubation times are not used. The most commonly used method for quantitative ethanol measurements in fermentation liquor is gas chromatography (GC) [16] with flame ionization detection [17] or coupled with mass spectrometry [18]. Similar to HPLC, GC methods require sample preparation and potentially longer analysis times.

Compared to the analytical techniques mentioned above, Raman and infrared spectroscopy are distinct because, in principle, all analytes in the sample can be measured simultaneously and non-invasively. Fourier transform infrared spectroscopy (FT-IR) operated in attenuated total internal reflection (ATR) mode with partial least squares spectral analysis has been used to monitor the effects of chemical pretreatment on wood [19]. Near IR spectroscopy has been used to monitor the fermentation reaction of glucose solutions using 
multilinear regression [20] or partial least squares analyses [21]. In contrast to FT-IR methods, Raman spectroscopy is suitable for the analysis of aqueous samples without the use of ATR due to the small Raman cross section of water between 0 and $3000 \mathrm{~cm}^{-1}$ [22,23]. In general, Raman spectra have narrow peak widths for aqueous samples and cover a wide spectral range. This reduces spectral interference and makes direct spectral interpretation possible, without the need for multivariate statistics.

Raman spectroscopy methods have been developed for measuring glucose in different matrices, including: human sera [24], blood [25,26], and urine [27]. FT-Raman spectroscopy has been used for on-line monitoring of the enzymatic hydrolysis of starch [28], and measuring structure changes in wheat upon different enzymatic treatments [29]. The fermentation of pure glucose solutions (i.e., not derived from biomass) at ambient temperatures [30] or at high pressures [31] has been measured by dispersive Raman spectroscopy using $514.5 \mathrm{~nm}$ incident light. High fluorescence background values are often observed using visible incident wavelengths.

Near IR incident lasers have become a popular choice for Raman spectroscopy measurements of biological samples, including plant materials. The low incident energy fluxes of near IR light can minimize both sample damage and fluorescence background that is problematic for most biological samples. Using a near IR spectrometer and a sensitive CCD camera, it is possible to obtain dispersive Raman spectra of most analytes with high sensitivity. Dispersive Raman spectroscopy using a 780nm diode laser has been reported for the fermentation of pure glucose solutions [32]. This work required the use of chemometric methods for glucose quantification due to the low intensity Raman peaks that were obtained. 
Other reports have demonstrated the use of Raman spectroscopy in conjunction with other spectroscopy techniques for monitoring hydrolysis [33] or fermentation reactions [34,35].

To our knowledge, there is no report of measuring the entire conversion process starting from biomass and ending with ethanol using Raman spectroscopy. Since pretreatment and hydrolysis byproducts can inhibit subsequent fermentation reactions, simply measuring the final ethanol yield does not provide a complete picture of ways to improve the conversion process. Measurements of the major product at each step in ethanol production are desirable. Herein, a Raman spectroscopy method for the determination of glucose and ethanol in biomass hydrolysis and fermentation reactions is described. Raman spectroscopy is ideal for monitoring reaction yield throughout the entire conversion process, and is particularly suited for screening many biomass types and conversion methods. The prototypical biomass sample used in this study is wild-type corn stover. Using a home-built Raman spectrometer, the effects of pretreatment conditions and spectral parameters on accurate glucose and ethanol quantification has been determined.

\section{Materials and methods}

\subsection{Materials}

All chemicals were reagent grade. Sodium acetate trihydrate, and acetic acid (glacial) were purchased from Fisher Scientific (Pittsburgh, PA). Cycloheximide, tetracycline (Fluka), d-glucose, microcrystalline cellulose and glucose HK reagent were purchased from SigmaAldrich (St. Louis, MO). Accellerase $1000^{\mathrm{TM}}$ enzyme was provided by Genencor (Rochester, NY). Ethanol TT yeast was provided by SPL International (Cheshire, United Kingdom). Yeast extract was purchased from Acros Organics (Geel, Belgium) and molecular biology- 
grade peptone was purchased from U.S. Biological (Swampscott, MA). All solutions were

prepared using ultrapure deionized (DI) water $\left(18.2 \mathrm{M} \Omega \mathrm{cm}^{-1}\right)$ from an Ultrapure II water system (Thermo Scientific, Waltham, MA).

\subsection{Raman spectroscopy instrumentation}

A home-built Raman microspectroscopy instrument that consisted of a 785-nm diode laser directed on to the rear port of a Nikon inverted microscope with a $40 \times$ magnification, 1.0 numerical aperture objective was used for all measurements. The collected signal was sent to a Holospec VPH spectrometer (Kaiser Optical System, Ann Arbor, MI) and a near IR enhanced PIXIS CCD camera (Princeton Instruments, Trenton, NJ) detected the scattered signal. A focusing lens and a $100 \mu \mathrm{m}$ pinhole (Edmund Optics, NJ) were positioned between the microscope and the spectrometer. As noted where appropriate in the figure legend, a fiber optic (RoMack Inc.VA) was used in place of the pinhole. No internal standard was used in these studies. The instrument was calibrated prior to each use with a standard acetonitrile/toluene mixture. This was both for wavelength calibration and to measure the intensity of the collected Raman scatter, which varies with changes in the laser power, instrument alignment, or sample volume. If these parameters cannot be accounted for by other means, use of an internal standard is advisable.

\subsection{Pretreatment of biomass by dilute acid}

Dried and ground biomass was weighed and placed in a $100-\mathrm{mL}$ beaker. Dilute sulfuric acid (1.0\%) in water was added in an amount of $20 \mathrm{~mL}$ per $1 \mathrm{~g}$ biomass. The beaker was covered and the solution was stirred on a magnetic stir plate for at least $4 \mathrm{~h}$. Each sample was then filtered with WhatmanTM No. 1 filter paper and rinsed with DI water until the pH of the rinse water was 6 , or until 2.0 L of water was used. Samples must be kept damp during 
and after filtration as fully drying the pretreated biomass samples was detrimental to subsequent hydrolysis reactions. Samples were stored in capped glass vials at $4{ }^{\circ} \mathrm{C}$ if shortterm storage was required.

\subsection{Pretreatment of biomass by ammonia}

Dried and ground biomass was weighed and placed in a 250-mL Erlenmeyer flask. A $15 \%(\mathrm{w} / \mathrm{w})$ ammonia/water solution was added to the flask in a ratio of $12 \mathrm{~mL}$ per $1 \mathrm{~g}$ biomass. The flask was placed in a $55{ }^{\circ} \mathrm{C}$ water bath and stirred for $36 \mathrm{~h}$. Hydrochloric acid (aq., $5 \mathrm{~N}$ ) was added until the $\mathrm{pH}$ was approximately 5. The pretreated biomass was then collected by centrifugation (Sorvall Biofuge PrimoR, Thermo Scientific) at $2525 \times \mathrm{g}$ for 60 min. DI water was added to the biomass and the solution was mixed for 3min using a shaker, followed by centrifugation to remove the wash water. Thiswashstepwasrepeated six times in order to remove soluble pretreatment by-products that inhibit subsequent reactions. Pretreated biomass was stored in a glass vial at $4{ }^{\circ} \mathrm{C}$ if short-term storage was required prior to initiating the hydrolysis reaction.

\subsection{Enzymatic hydrolysis reactions}

Enzymatic hydrolysis reactions were carried out in $0.1 \mathrm{M}$ acetate buffer, $\mathrm{pH} 4.8$ with addition of antibiotics. For the cellulose reaction, approximately 0.35 g cellulose was placed in a 25-mL Erlenmeyer flask followed by the addition of $0.20 \mathrm{~mL}$ Accellerase 1000TM enzyme, $200 \mu \mathrm{L}$ cycloheximide, $30 \mu \mathrm{L}$ tetracycline $\left(10 \mathrm{~g} \mathrm{~L}^{-1}\right.$ in $70 \%$ ethanol) and $6.8 \mathrm{~mL}$ acetate buffer. For the biomass reaction, approximately $14 \mathrm{~g}$ of wet pretreated corn stover was placed in a $25-\mathrm{mL}$ Erlenmeyer flask followed by the addition of $0.80 \mathrm{~mL}$ Accellerase $1000^{\mathrm{TM}}$ enzyme, $200 \mu \mathrm{L}$ cycloheximide, $30 \mu \mathrm{L}$ tetracycline and $10 \mathrm{~mL}$ acetate buffer. Standards for constructing the calibration curves were prepared using glucose in place of the 
corn stover/cellulose in the same matrix. Capped flasks were placed in an incubated shaker at $50{ }^{\circ} \mathrm{C}, 150 \mathrm{rpm}$ for $48 \mathrm{~h}$. The samples were heated to $60{ }^{\circ} \mathrm{C}$ to terminate the enzymatic reaction at the end of the incubation period. Samples were then centrifuged at approximately $6200 \times \mathrm{g}$ for $20 \mathrm{~min}$ and the supernatants were filtered using a $0.22 \mu \mathrm{m}$ filter.

\subsection{Fermentation reactions}

A 10× YP medium was prepared by dissolving $10.0 \mathrm{~g}$ yeast extract and $20.0 \mathrm{~g}$ peptone in $100 \mathrm{~mL}$ deionized water followed by autoclaving at $121{ }^{\circ} \mathrm{C}$ for $30 \mathrm{~min}$. For the glucose fermentation, a mixture of $1.0 \mathrm{~mL} \mathrm{pH} 4.8$ acetate buffer, $1.0 \mathrm{~mL}$ of 10× YP, $3.0 \mathrm{~mL}$ glucose solution, $0.02 \mathrm{~g}$ of ethanol TT yeast and $5.0 \mathrm{~mL}$ autoclaved water was added to the 20-mL vial. For the fermentation of hydrolysate, a mixture of $1.0 \mathrm{~mL} \mathrm{pH} 4.8$ acetate buffer, $1.0 \mathrm{~mL}$ of $10 \times \mathrm{YP}, 6.0 \mathrm{~mL}$ hydrolysate containing $244 \mathrm{~g} \mathrm{~L}^{-1}$ glucose, $0.02 \mathrm{~g}$ of ethanol TT yeast and $2.0 \mathrm{~mL}$ autoclaved water was added to the $20-\mathrm{mL}$ vial. The vial had a $30-\mathrm{mm}$ diameter hole punched through a septum in the cap. Tygon ${ }^{\mathrm{TM}}$ tubing was connected to a flask through the hole on one side and was immersed in a $1 \%$ sulfuric acid solution on the other side to allow carbon dioxide to escape while maintaining an anaerobic environment. The entire assembly was then placed in an incubated shaker set at $32{ }^{\circ} \mathrm{C}, 130 \mathrm{rpm}$ for $48 \mathrm{~h}$.

\subsection{Raman spectroscopy analysis}

Approximately $1.4 \mathrm{~mL}$ of the standard or unknown solution was loaded into a sample holder, which was fabricated with a glass coverslip bottom and Teflon walls. The Raman scatter was collected using $10 \mathrm{~s}$ integrations with 20 accumulations for glucose quantification, and $10 \mathrm{~s}$ integrations with 10 accumulations for ethanol quantification, unless

otherwise noted in the figure legend. All measurements were taken at room temperature. The laser power for all measurements was approximately $130 \mathrm{~mW}$ at the sample. 


\subsection{Spectral analysis and statistical analysis}

Spectra were collected using the program Winspec32 (Roper Scientific, Germany) and processed using the software Igor Pro 4.0 (WaveMetrics. Inc, OR) to perform background subtraction and for the bottom spectra in Fig. 2 only, baseline correction. The following spectra were used for the background: spectrum of the hydrolysis matrix with deactivated enzyme for the glucose standard solutions; spectrum of the enzymatic hydrolysis matrix with cellulose or biomass without enzyme for the hydrolysis samples; spectrum of fermentation liquor for the ethanol standard solutions; or spectrum of the fermentation broth minus glucose/biomass for the fermentation samples. The analyte concentration was determined using a calibration curve, which was constructed using partial least square

regression. All calibration curves have coefficients of determination $\left(\mathrm{R}^{2}\right)$ greater than 0.99 , indicating the concentration can be predicted by the model. Minimum detectable concentration (detection limit) was calculated by analyzing seven replicate samples containing glucose or ethanol at appropriate concentrations, and computing three times the standard deviation of the replicate measurements divided by the slope of calibration curve (Supplemental Figs. 2B or 4B) [36]. Means were compared using Student’s t-tests.

\section{Results and discussion}

\subsection{Optimization of glucose measurements in hydrolysis liquor using Raman spectroscopy}

Two biomass pretreatment methods were investigated in this study in order to reveal the influence of lignin content on subsequent Raman analyses. Ammonia and dilute acid pretreatment were chosen from numerous choices as model lignin retaining or lignin extracting pretreatments. Ammonia pretreatment removes up to $85 \%$ of the lignin while 
dilute acid pretreatment removes only $30 \%$ of the lignin. The effects of the pretreatment step on subsequent Raman spectroscopic analyses will be discussed in further detail below.

Following pretreatment, enzymatic hydrolysis was performed. The commercial enzyme mixture used in this study is Accelerase ${ }^{\mathrm{TM}} 1000$, which contains enzymes with exoglucanase, endoglucanase, hemicellulase and beta-glucosidase activity [37]. This enzyme mixture was derived from a genetically modified strain of Trichoderma reesei. Other enzymes that we have been successfully employed with the Raman spectroscopic method outlined herein were wildtype cellulase mixtures from Trichoderma reesei and Trichoderma viride.

In determining the amount of glucose generated from the enzymatic hydrolysis of biomass, polysaccharides and other monosaccharides posed the greatest possibility of spectral interference. These include cellulose, hemicellulose and hemicelluloses hydrolysis products. The Raman spectra of cellulose and glucose, obtained under identical conditions, are shown in Fig. 2 (top and middle). For all glucose spectra, the spectral range plotted is from 350 to $1500 \mathrm{~cm}^{-1}$. No peaks were observed between 1500 and $1700 \mathrm{~cm}^{-1}$, the upper limit for the spectrometer grating. The Raman cross section of glucose is significantly larger than that of cellulose; therefore, only minimal spectral contribution from low concentrations of cellulose is expected. Due to limited solubility of cellulose in aqueous solutions, low cellulose concentrations can be achieved by filtration or centrifugation prior to analysis using Raman spectroscopy. A Raman spectrum of cellulose taken with a longer acquisition time (Fig. 2, bottom) revealed unique peaks that can be used to distinguish cellulose from glucose spectrally. Hemicellulose is a weak Raman scatterer, and Raman spectra of biomass showed no spectral contributions that could be attributed to hemicellulose in the presence of cellulose 
[38]. The possibility of spectral interference by hemicellulose hydrolysis products is discussed below.

Spectral acquisition parameters were optimized to achieve a low glucose detection limit in hydrolysate (Supplemental Fig. 1). The optimum spectral parameters yielded a glucose detection limit in hydrolysis liquor of $8 \mathrm{~g} \mathrm{~L}^{-1}$. A range of hydrolysis efficiencies for different biomass types and pretreatment steps was 56-92\% [6,9]. Using the reaction conditions reported in Section 2.5, the expected range of glucose concentrations relevant for hydrolysis of biomass was from 41 to $68 \mathrm{~g} \mathrm{~L}^{-1}$. This is well above the detection limit that can be achieved by Raman spectroscopy. Considering the measured detection limit, glucose in corn stover hydrolysate can be quantified when the hydrolysis efficiency is as low as $10.8 \%$ using this method. The detection limit for other biomass types will be similar to this value, but will depend on the starting cellulose composition of the biomass. Corn stover is approximately $37 \%$ cellulose. Switchgrass is approximately $35 \%$ cellulose and the lowest quantifiable hydrolysis efficiency will be $11.4 \%$; whereas, sugar cane bagasse is approximately 39\% cellulose and 10.3\% hydrolysis efficiencies will be measurable [39].

The spectra shown in Fig. 3 (top and middle) are the background subtracted Raman spectra of the enzymatic hydrolysis liquor with a starting reactant of pure cellulose powder or corn stover, respectively. The spectra were acquired after termination of the hydrolysis reaction by increasing the incubator temperature to $60{ }^{\circ} \mathrm{C}$ for $10 \mathrm{~min}$, and the remaining particulates were removed by centrifugation and filtration. Although heating the solution to deactivate the enzyme was not required for Raman spectroscopic analyses, this step was performed to ensure there was no change in glucose concentration between successive measurements of the same sample. 
Other components in the enzymatic hydrolysis liquor were acetate buffer, antibiotics, enzymes, and soluble biomass compounds. In order to obtain a representative background signal, a control experiment containing all the components of the hydrolysis reaction including deactivated enzyme was used for background subtraction. A spectrum of $67 \mathrm{~g} \mathrm{~L}^{-1}$ glucose is shown in Fig. 3 (bottom) to compare to the spectra from the hydrolysis liquor (Fig. 3, top and middle). The presence of glucose in both hydrolysis liquors was confirmed with similar yields for the cellulose and biomass reactions. There were two minor differences in the spectrum of corn stover hydrolysate compared to the glucose standard: a smaller peak at $449 \mathrm{~cm}^{-1}$ and an additional peak at $536 \mathrm{~cm}^{-1}$. These differences may indicate the presence of other soluble molecules derived from the plant cell wall, which would not be present in the cellulose reaction. These peaks were not used for subsequent quantification steps.

\subsection{Role of biomass pretreatment in enzymatic hydrolysis and Raman spectra}

As discussed above, pretreatment steps can disrupt the lignin-hemicellulose/cellulose linkage, and the lignin can either be left in the plant cell wall or be extracted. Lignin contributed a relatively large background (Fig. 4, top) even with the $785 \mathrm{~nm}$ near IR incident wavelength used in this study. This background signal interfered with glucose quantification using Raman spectroscopy. The spectra from the enzymatic hydrolysis liquors for corn stover that has been pretreated with dilute acid (middle) or ammonia (bottom) are also shown in Fig. 4. The reaction liquor for the dilute acid-pretreated biomass had a large background, likely from soluble lignin species that remained in the cell wall after pretreatment. Glucose quantification by Raman spectroscopy using $785 \mathrm{~nm}$ light was not possible with the acidpretreated corn stover. In comparison, ammonia had been reported to remove up to $85 \%$ of lignin [6], and the glucose spectral peaks were easily observed in the hydrolysis broth for the 
ammonia-pretreated corn stover. These results indicate that decreasing the lignin concentration during the pretreatment step was a requirement for subsequent analysis of hydrolysis reactions when $785 \mathrm{~nm}$ Raman spectroscopy is used. Longer wavelengths of incident light could be used to reduce the background, but this would result in a concomitant decrease in Raman signal and background. Ammonia pretreatment effectively reduces lignin in the cell wall; however, approximately $15 \%$ of lignin remained in the pretreated corn stover. Quantification of the amount of lignin that must be removed in order to accurately measure glucose using Raman spectroscopy is on-going.

It is well established that lignin inhibits hydrolysis and fermentation reactions $[4,6]$. Using glucose HK assays to measure glucose in the reaction liquor, it was determined that ammonia pretreatment significantly increases the hydrolysis efficiency (78\%) compared to acid-pretreated biomass (40\%). Thus pretreatment steps that reduced lignin content not only facilitated Raman spectroscopic analysis, but also increased the glucose yield. Other pretreatments steps that extract lignin from the cell wall, such as pretreatment with calcium hydroxide [40], acid-catalyzed steam [41], alkali [42] and alkaline peroxide [43] should be suited for Raman spectroscopy analysis.

\subsection{Enzymatic hydrolysis and glucose quantification}

To validate Raman spectroscopy as a method for measuring saccharification efficiency, a calibration curve using glucose in the same matrix as the hydrolysis sample was used (Supplemental Fig. 2). Standard glucose solutions ranging from 17 to $200 \mathrm{~g} \mathrm{~L}^{-1}$ were used, and each calibration point was measured in triplicate. Calibration curves were generated from several glucose spectral features. All the calibration curves were suitable for quantitative glucose determination in the hydrolysis liquor when the starting material was 
pure cellulose. For the biomass samples, a calibration curve constructed by subtracting the intensity of the $1128 \mathrm{~cm}^{-1}$ peak by the intensity of the baseline at $1090 \mathrm{~cm}^{-1}$ provided the best glucose detection limit, produced the most linear calibration curve (Supplemental Fig. 2), and resulted in glucose concentrations that most closely matched data from glucose HK assays (Table 1).

Using the developed calibration curve, the amount of glucose in the enzymatic hydrolysis liquors for three cellulose and three corn stover samples was calculated (Table 1). The cellulose composition of the corn stover is approximately $37 \mathrm{~g}$ per $100 \mathrm{~g}$ biomass. Using this starting composition, and the conversion of $1 \mathrm{~g}$ cellulose to $1.1 \mathrm{~g}$ glucose, the theoretical glucose yield was calculated. Comparing the actual glucose yield, measured by Raman spectroscopy, saccharification efficiencies were calculated. The average of three replicate experiments yielded similar efficiencies of approximately $85 \%$ and $86 \%$ for the cellulose and biomass samples, respectively.

A glucose HK assay calibration curve (Supplemental Fig. 3) was used to determine the glucose concentration in the same hydrolysis mixtures measured by Raman spectroscopy (Table 1). The average of three replicate experiments falls within the $95 \%$ confidence interval for both the cellulose and corn stover hydrolysis liquors. The fact that the quantification by Raman spectroscopy and the glucose specific HK assay agree for the cellulose hydrolysis reaction suggests that partial cellulose hydrolysis products (e.g., cellobiose) does not interfere with the spectral measurements of glucose using this protocol.

The two independent measurements for corn stover hydrolysate fell within the 95\% confidence interval, but the average of the Raman spectroscopy hydrolysis efficiency was $10 \%$ higher than the average measured by the glucose HK assay. It could be argued that 
hydrolysis products from residual hemicellulose spectrally interfered the wavelengths used to construct the Raman spectroscopy calibration curve for glucose. These sugars would not be measured in the specific glucose HK assay, so the glucose HK value would be lower than the Raman measurement. However, it is not likely that hemicellulose-derived sugars were at sufficient concentration to spectrally interfere with glucose for the following reasons. First, almost $60 \%$ of the hemicellulose was removed during the ammonia pretreatment step, and was present at one-third the weight of cellulose. Second, the hemicellulase present in the enzyme mixture is not present at sufficient diversity or concentration to yield a significant amount of simple sugars from hemicellulose to interfere with the glucose measurement [44].

Future generations of enzyme mixtures will contain more diverse and concentrated hemicellulases to completely hydrolyze the hemicellulose and increase overall ethanol yields. Once these mixtures are available and commonly used, a single sugar monomer can be measured with multivariate statistical analysis on the more complex spectra of the hydrolysis liquor [32]. Enzymatic assays will require a separate enzyme reaction for each sugar that is measured. In addition, the precision of the Raman spectroscopy measurements was better than that of the glucose HK measurements. The enzymatic assays required an incubation step which caused variations in the UV-Vis spectrophotometry reading with slight differences in incubation time, despite efforts to keep incubation times as close to $20 \mathrm{~min}$ as possible. The limited shelf life of the glucose HK reagent was also a concern regarding the reproducibility for this assay method.

\subsection{Fermentation and ethanol quantification}

The second step in biomass conversion to ethanol was a fermentation reaction. The addition of yeast and growth medium to the hydrolysate yielded a more complex matrix. For 
ethanol measurements, the C-H stretching mode peaks at 2878 and $2972 \mathrm{~cm}^{-1}$ have a higher Raman cross section than the $\mathrm{C}-\mathrm{C}$ stretching mode at $883 \mathrm{~cm}^{-1}$. However, the near IR enhanced detector used in this study has a low quantum efficiency at the C-H spectral region. As a consequence, better detection limits were obtained using the $883 \mathrm{~cm}^{-1}$ peak. Additionally, this ethanol peak had no spectral interference with any glucose peaks in the Raman spectroscopy measurements.

In comparison to glucose, ethanol has a fairly large Raman cross section and can be identified down to a concentration of $6 \mathrm{~g} \mathrm{~L}^{-1}$ in the fermentation broth with optimized acquisition parameters (Supplemental Fig. 4). Typical fermentation efficiencies are in the range of $76-83 \%$ [8,32], and $1.0 \mathrm{~g}$ of glucose is converted to $0.51 \mathrm{~g}$ ethanol [45]. Using the acquisition parameters reported here and a $76 \%$ fermentation efficiency, ethanol quantification is possible as long as the starting glucose concentration is $15.5 \mathrm{~g} \mathrm{~L}^{-1}$ or higher in the fermentation liquor.

Fermentation reactions using pure glucose or hydrolysate were performed to test the ability to measure ethanol yield by Raman spectroscopy. The ammonia-pretreated biomass hydrolysate was used since the high background from the dilute acid-pretreated biomass carries over to the fermentation reaction. After filtering the residual particulates from the fermentation reaction of a glucose or enzymatic hydrolysate solution (Fig. 5 top and middle) yielded a Raman spectrum characteristic of ethanol (Fig. 5, bottom). The spectra were background subtracted as described in Section 2.8.

Raman spectroscopy can be used to measure ethanol yield in the fermentation reaction of glucose and corn stover hydrolysate (Table 2). Three replicate experiments were performed for glucose solutions and the corn stove hydrolysate. The theoretical ethanol yield 
was calculated from the starting glucose concentration as measured by Raman spectroscopy and the conversion $1 \mathrm{~g}$ glucose/0.51 $\mathrm{g}$ ethanol. Using the Raman spectroscopy calibration curve for ethanol (Supplemental Fig. 4), the ethanol yield was measured for each solution. The fermentation efficiency was the ratio of the actual to theoretical ethanol yield. The fermentation efficiency for converting glucose to ethanol was measured to be approximately $77 \%$ for a glucose standard and $84 \%$ for the corn stover hydrolysate. These values were validated using head space gas chromatography-mass spectrometry (Table 2). The Student's t-test was performed to confirm that the results for the two analysis methods agreed within the 95\% confidence interval for three replicate experiments. These results highlighted that Raman spectroscopy can be used to measure ethanol yield and biomass fermentation efficiency.

\section{Conclusions}

Dispersive Raman spectroscopy with $785 \mathrm{~nm}$ incident light can be used to monitor the conversion efficiency for hydrolysis and fermentation of biomass samples without spectral interference or high background values if a pretreatment that sufficiently reduces the lignin concentration is used. The measurement is sensitive and can measure glucose and ethanol concentrations in complex matrices at concentrations relevant to biomass conversion reactions without enhancement mechanisms such as surface enhance Raman spectroscopy. This protocol requires minimal sample preparation and analysis time, and can be automated. With only seconds to minutes required for each measurement and minimal spectral processing required, Raman spectroscopy is ideally suited for screening different biomass types, growing conditions, or reaction conditions. The developed Raman spectroscopic 
method also has potential for utilizing fiber optics for remote monitoring at different locations in the production facility. For example, in-reactor measurements would be possible if the reactions were filtered prior to illumination with an incident laser beam. Flow control and on-line filtration methods that can be incorporated into the reaction chamber have been previously described [32]. Collaborative work is underway to screen additional biomass feedstocks and conditions for increased ethanol production.

\section{Acknowledgements}

Funding for this research was provided by the Iowa State University Plant Sciences Institute and Ames Laboratory, which is operated for the U.S. Department of Energy by Iowa State University under Contract DE-AC02-07CH11358. The authors thank Erin Bilsten and Stephen Fosdick for their help in preparing samples, Dr. Kenneth J. Moore for providing the

corn stover, Genecor International for providing Accelerase ${ }^{\mathrm{TM}}$ 1000, and SPL International for providing the ethanol TT yeast.

\section{References}

[1] U.S. DOE, http://www.eia.doe.gov/cneaf/solar.renewables/page/trends/table1.html, 2008.

[2] M. Knauf, M. Moniruzzaman, Int. Sugar J. 106 (2004) 147.

[3] T. Koshijima, T. Watanabe, Association Between Lignin and Carbohydrates in Wood, Springer-Verlag, Berlin Heidelberg, New York, 2003.

[4] V. Méchin, O. Argillier, F. Rocher, Y. Hébert, I. Mila, B. Pollet, Y. Barriére, C. Lapierre, J. Agric. Food Chem. 53 (2005) 5872. 
[5] N. Mosier, C. Wyman, B. Dale, R. Elander, Y.Y. Lee, M. Holtzapple, M. Ladisch, Bioresour. Technol. 96 (2005) 673.

[6] T.H. Kim, J.S. Kim, C. Sunwoo, Y.Y. Lee, Bioresour. Technol. 90 (2003) 39.

[7] T.H. Kim, Y.Y. Lee, Appl. Biochem. Biotechnol. 136-140 (2007) 81.

[8] K. Öhgren, J. Vehmaanperä, M. Siika-Aho, M. Galbe, L. Viikari, G. Zacchi, Enzyme Microb. Technol. 40 (2007) 607.

[9] T.A. Lloyd, C.E. Wyman, Bioresour. Technol. 96 (2005) 1967.

[10] A. Sluiter, B. Hames, R. Ruiz, C. Scarlata, J. Sluiter, D. Templeton, Laboratory Analytical Procedure, National Renewable Energy Laboratory, Golden, CO, USA, 2006.

[11] R. Raymond, T. Ehrman, Laboratory Analytical Procedure (LAP-013), National Renewable Energy Laboratory, Golden, CO, USA, 1996.

[12] F.A. Agblevor, B.R. Hames, D. Schell, H.L. Chum, Appl. Biochem. Biotechnol. 136 (2007) 309.

[13] H. Tatsumi, H. Katano, Chem. Lett. 33 (2004) 692.

[14] C. Cara, E. Ruiz, J.M. Oliva, F. Sáez, E. Castro, Bioresour. Technol. 99 (2008) 1869.

[15] R.J. Bondar, D.C. Mead, Clin. Chem. 20 (1974) 586.

[16] V. Diamantis, P. Melidis, A. Aivasidis, Anal. Chim. Acta 573-574 (2006) 189.

[17] H. Li, X.-S. Chai, Y. Deng, H. Zhan, S. Fu, J. Chromatogr., A 1216 (2009) 169.

[18] J.C. Motte, X. Monseur, M. Termonia, M. Hofman, G. Alaerts, A. De Meyer, P. Dourte, J. Walravens, Anal. Chim. Acta 163 (1984) 275.

[19] M.P. Tucker, R.K. Mitri, F.P. Eddy, Q.A. Nguyen, L.M. Gedvilas, J.D. Webb, Appl. Biochem. Biotechnol. 84-86 (2000) 39.

[20] A.G. Cavinato, D.M. Mayes, Z. Ge, J.B. Callis, Anal. Chem. 62 (1990) 1977. 
[21] M. Blanco, A.C. Peinado, J. Mas, Biotechnol. Bioeng. 88 (2004) 536.

[22] X. Zhang, M.A. Young, O. Lyandres, R.P. Van Duyne, J. Am. Chem. Soc. 127 (2005) 4484.

[23] M.H. Brooker, G. Hancock, B.C. Rice, J. Shapter, J. Raman Spectrosc. 20 (1989) 683.

[24] J.Y. Qu, B.C. Wilson, D. Suria, Appl. Opt. 38 (1999) 5491.

[25] A.J. Berger, I. Itzkan, M.S. Feld, Spectrochim. Acta, Part A 53A (1997) 287.

[26] A.M.K. Enejder, T.-W. Koo, J. Oh, M. Hunter, S. Sasic, M.S. Feld, G.L. Horowitz, Opt. Lett. 27 (2002) 2004.

[27] D. Qi, A.J. Berger, Appl. Opt. 46 (2007) 1726.

[28] K.C. Schuster, H. Ehmoser, J.R. Gapes, B. Lendl, Vib. Spectrosc. 22 (2000) 181.

[29] Y. Cao, Y. Lu, Y. Huang, J. Mol. Struct. 693 (2004) 87.

[30] T.B. Shope, T.J. Vickers, C.K. Mann, Appl. Spectrosc. 41 (1987) 908.

[31] A. Picard, I. Daniel, G. Montagnac, P. Oger, Extremophiles 11 (2007) 445.

[32] A.D. Shaw, N. Kaderbhai, A. Jones, A.M. Woodward, R. Goodacre, J.J. Rowland, D.B. Kell, Appl. Spectrosc. 53 (1999) 1419.

[33] L. Laureano-Perez, B.E. Dale, J.P. O’Dwyer, M. Holtzapple, Biotechnol. Prog. 22 (2006) 835.

[34] S. Sivakesava, J. Irudayaraj, D. Ali, Process Biochem. 37 (2001) 371.

[35] S. Sivakesava, J. Irudayaraj, A. Demirci, J. Ind. Microbiol. Biotechnol. 26 (2001) 185.

[36] D.C. Harris, Quantitative Chemical Analysis, Craig Bleyer, New York, 2007.

[37] Genencor, Accellerace 1000, Cellulase Enzyme Complex for Lignocellulosic Biomass Hydrolysis, Rochester, NY, 2007.

[38] U.P. Agarwal, S.A. Ralph, Appl. Spectrosc. 51 (1997) 1648. 
[39] U.S. DOE, http://www.afdc.energy.gov/biomass/progs/search1.cgi, 2004.

[40] S. Kim, M.T. Holtzapple, Bioresour. Technol. 97 (2006) 778.

[41] K. Öhgren, R. Bura, J. Saddler, G. Zacchi, Bioresour. Technol. 98 (2007) 2503.

[42] P. Persson, J. Andersson, L. Gorton, S. Larsson, N.-O. Nilvebrant, L.J. Jönsson, J. Agric. Food Chem. 50 (2002) 5318.

[43] M.J. Selig, T.B. Vinzant, M.E. Himmel, S.R. Decker, Appl. Biochem. Biotechnol. 155 (2009) 397.

[44] Y. Lu, N.S. Mosier, Biotechnol. Prog. 23 (2007) 116.

[45] N. Dowe, J. McMillan, Laboratory Analytical Procedure (LAP-008), National Renewable Energy Laboratory, Golden, CO, USA, 2001. 

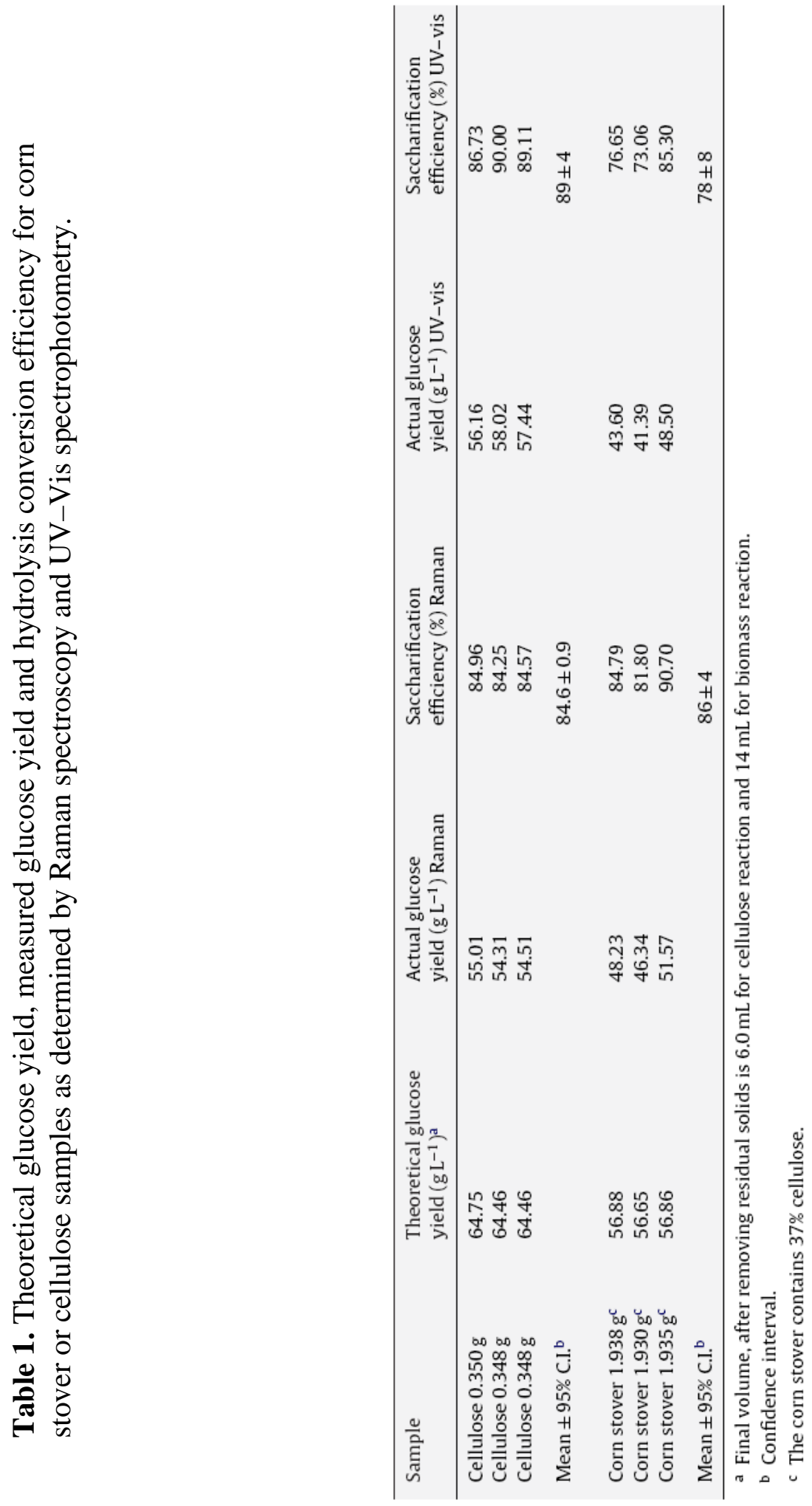

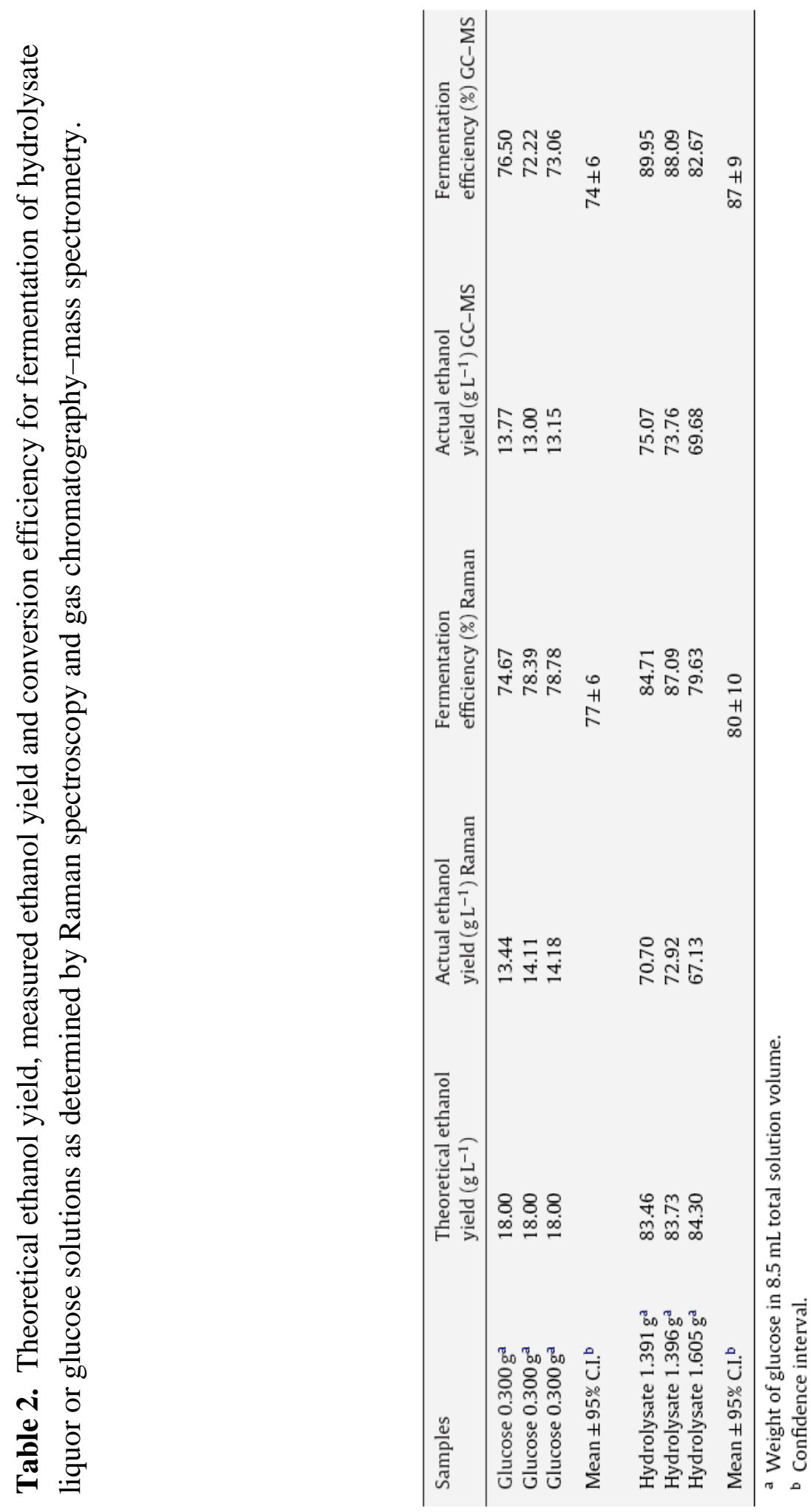


\section{Figure captions}

Figure 1: Schematic showing the steps to convert cellulosic biomass to ethanol. The biomass used in these studies is corn stover. After pretreatment, the cellulose is enzymatically hydrolyzed to glucose using a commercial enzyme mixture and then fermented to ethanol. Accurate glucose and ethanol quantification using Raman spectroscopy depends on the chosen pretreatment method as discussed in the text.

Figure 2: Background subtracted Raman spectra of (top) solid glucose with 20 s; (middle) cellulose with $20 \mathrm{~s}$; and (bottom) cellulose with $30 \mathrm{~s}$ acquisition times and 60 accumulations. The bottom spectrum was collected using a fiber optic in the collection path, for further details see the experimental section. Scale bar represents 12,000 counts for top and middle spectra and 1 count for bottom spectrum. Spectra are offset for clarity.

Figure 3: Background subtracted Raman spectra of the reaction liquor after the enzymatic hydrolysis of (top) $51.0 \mathrm{~g} \mathrm{~L}^{-1}$ cellulose; (middle) ammonia-pretreated corn stover containing approximately $51.2 \mathrm{~g} \mathrm{~L}^{-1}$ of cellulose; and (bottom) a $66.7 \mathrm{~g} \mathrm{~L}^{-1}$ standard glucose solution. For all spectra acquisition parameters are $10 \mathrm{~s}$ and 20 accumulations. Spectra are offset for clarity.

Figure 4: Background subtracted Raman spectra of (top) pure lignin; and the reaction liquor after the enzymatic hydrolysis of corn stover pretreated with (middle) sulfuric acid; or (bottom) ammonia. For all spectra acquisition parameters are $10 \mathrm{~s}$ and 20 accumulations. Scale bar represents (top) 84,000 counts; (middle) 12,000 counts and (bottom) 8000 counts.

Figure 5: Background subtracted Raman spectra after fermenting (top) corn stover hydrolysate containing $1391 \mathrm{mg}$ glucose; (middle) 300mg glucose and (bottom) a $25 \mathrm{~g} \mathrm{~L} \mathrm{~L}^{-1}$ standard ethanol solution prepared in fermentation liquor. For all spectra acquisition parameters are $10 \mathrm{~s}$ acquisition and 10 accumulations. 


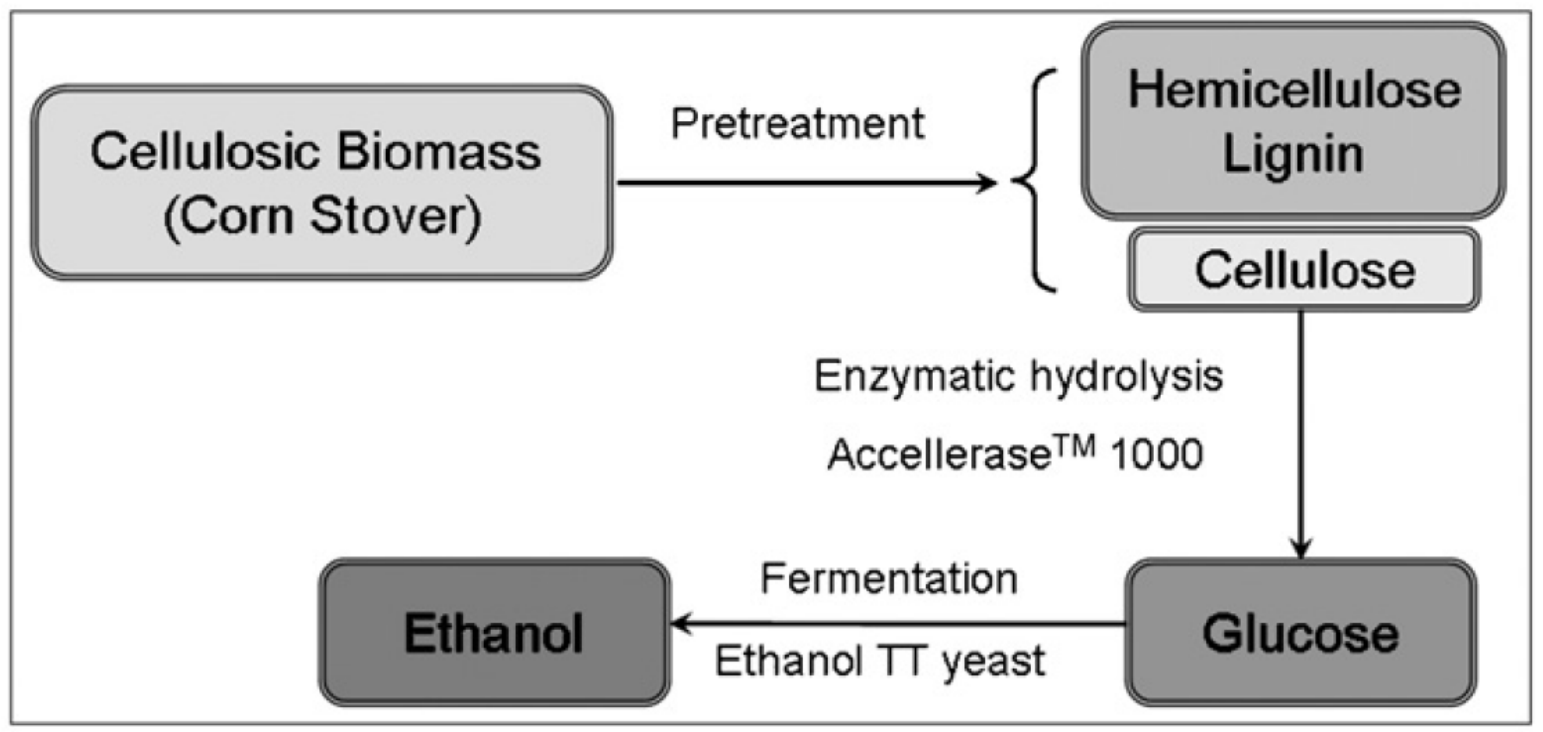

Figure 1. 


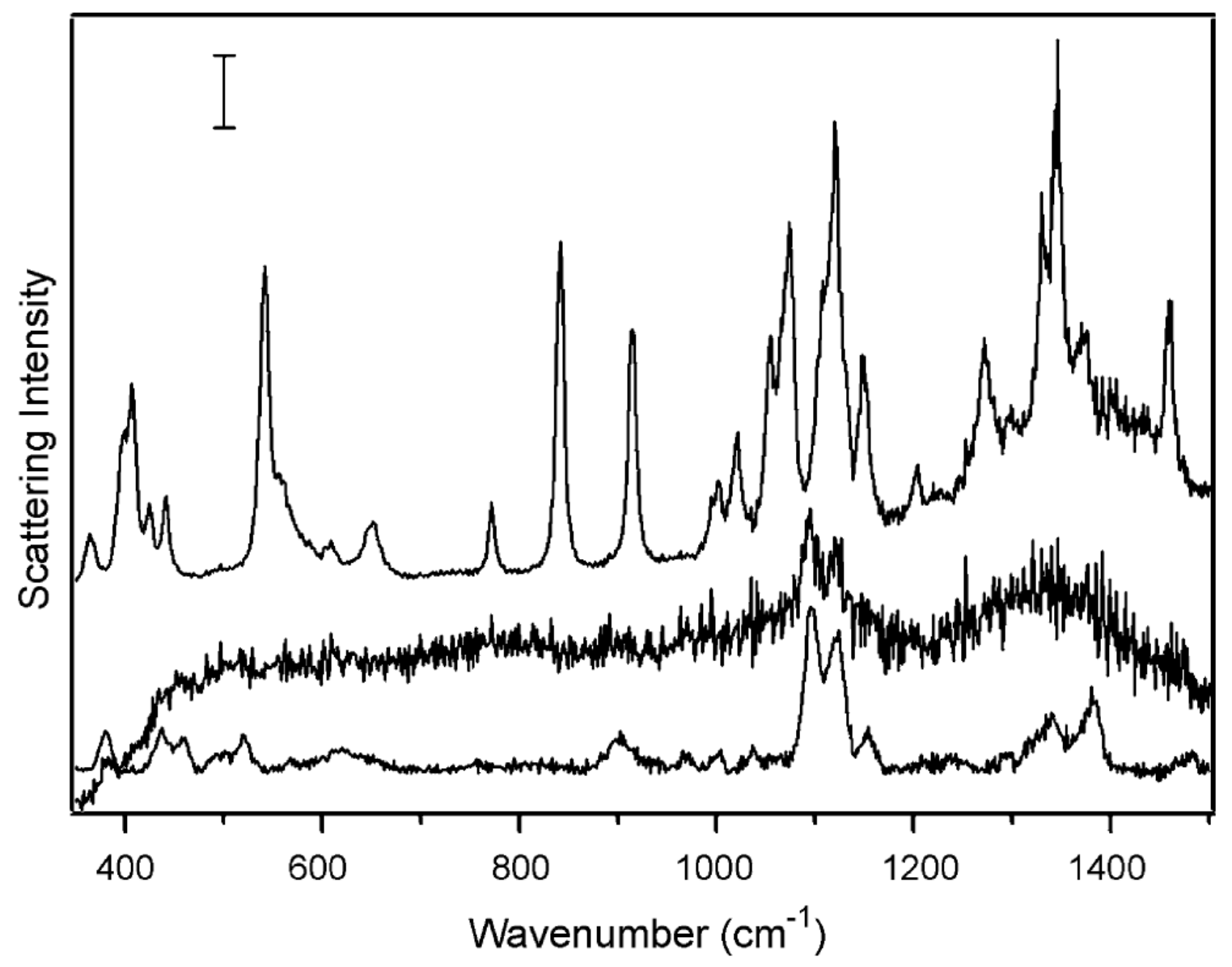

Figure 2. 


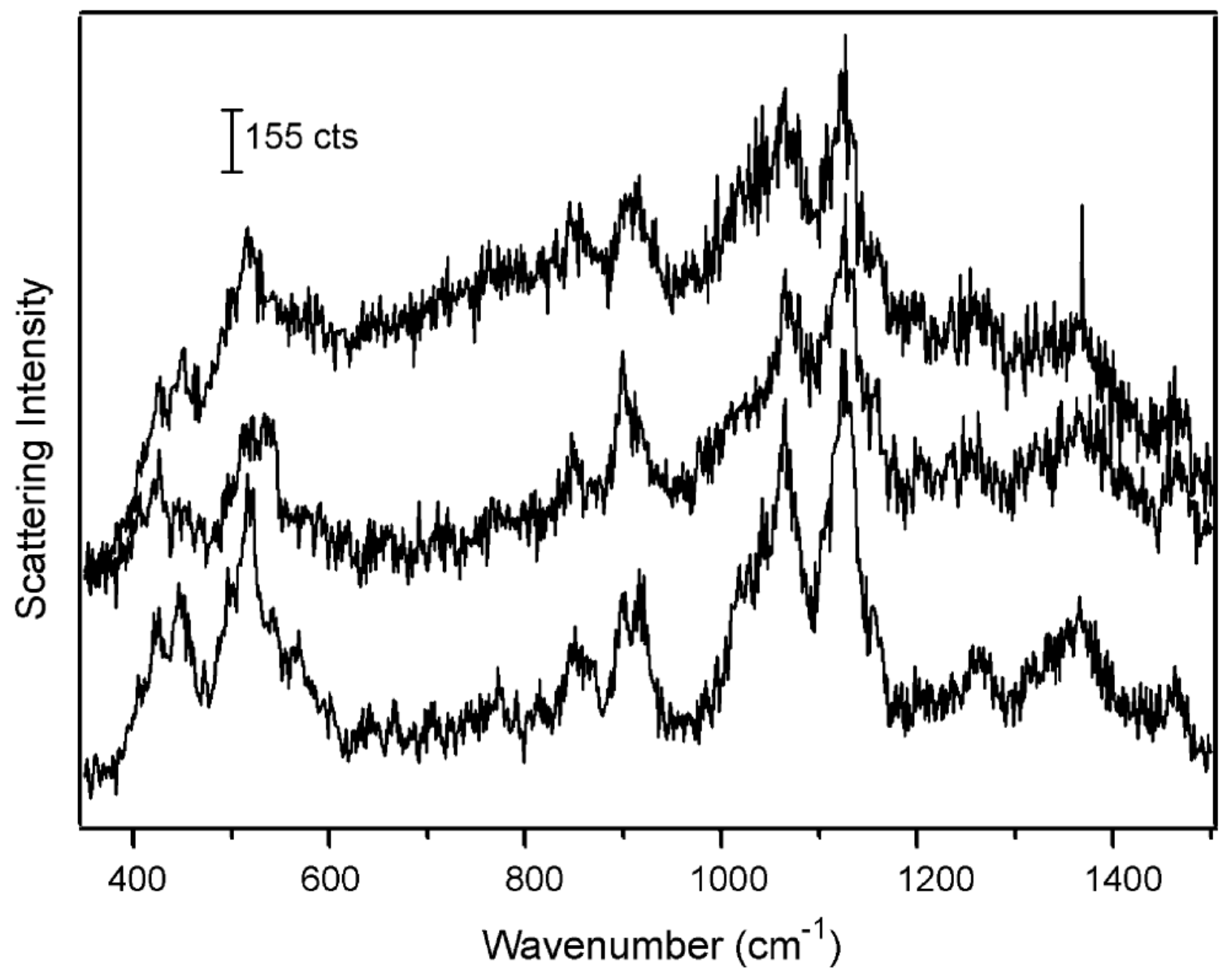

Figure 3. 


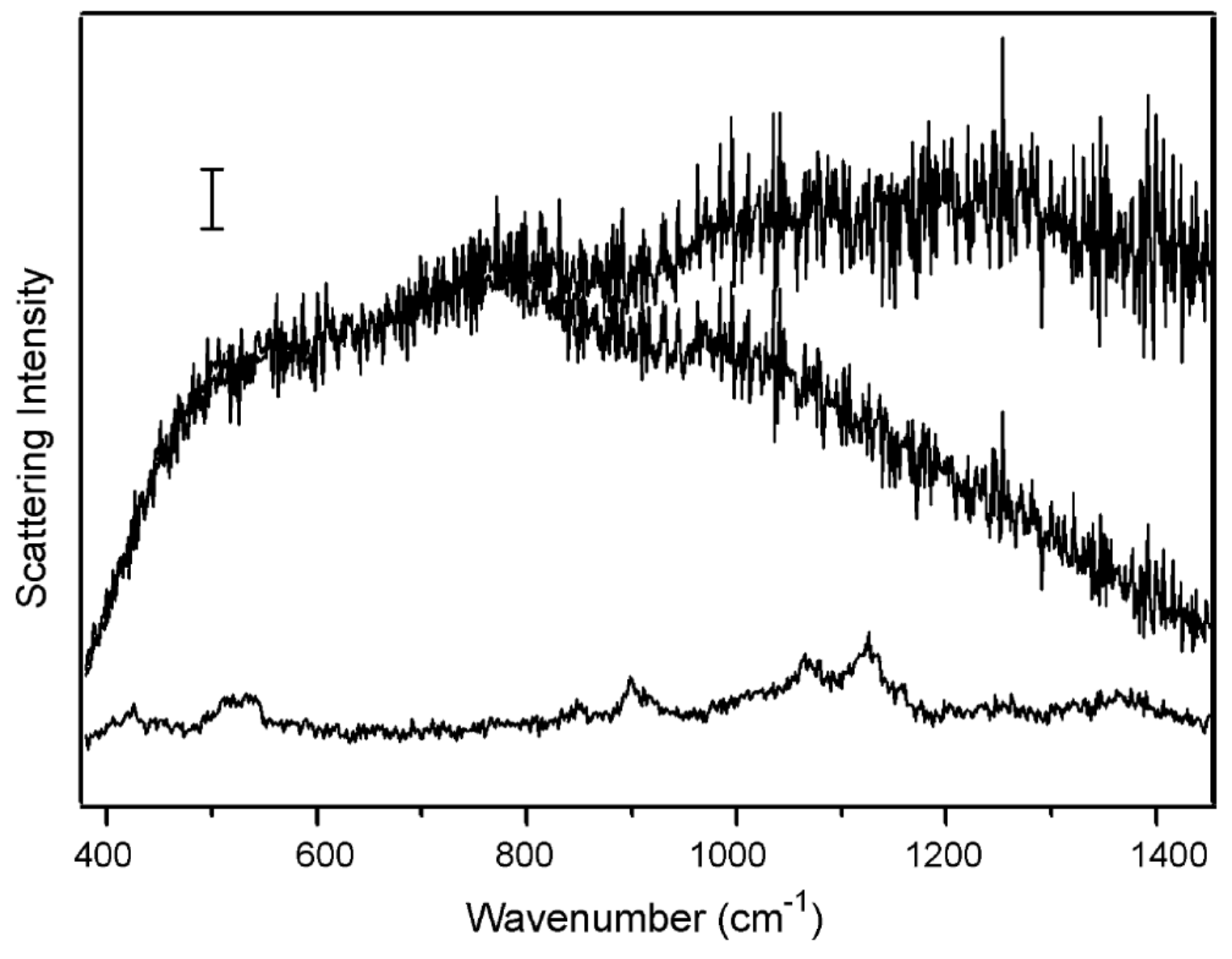

Figure 4. 


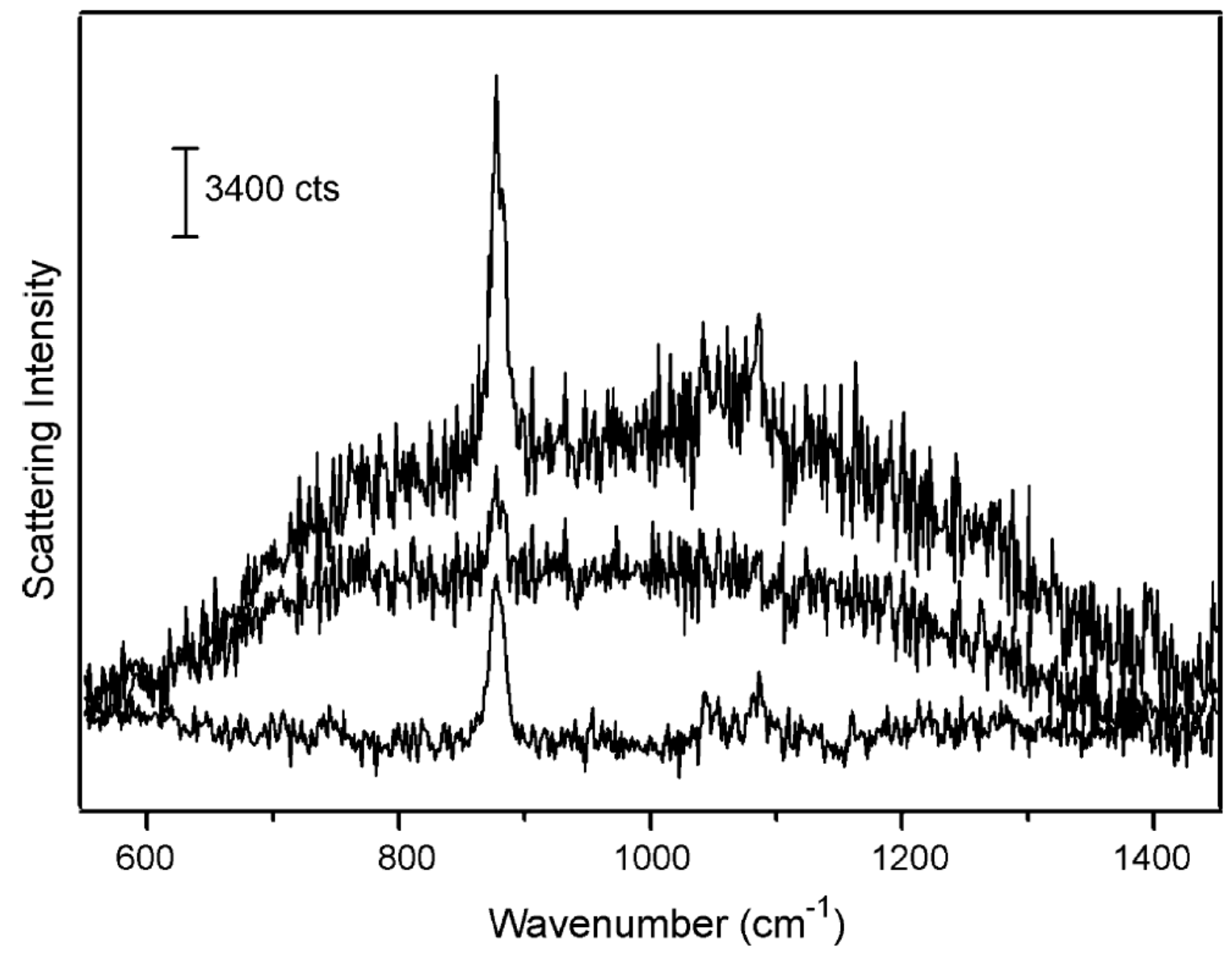

Figure 5. 


\title{
Supplemental materials and methods
}

\author{
Quantification of glucose using UV-Vis spectrophotometry
}

To determine the glucose concentration using a glucose HK assay, the protocol suggested by the manufacturer was followed (Sigma Aldrich). This assay is also discussed in reference: R.J. Bondar, D.C. Mead, Clin. Chem., 20 (1974) 586. Briefly, the hydrolysis sample was diluted to approximately 1 to $5 \mathrm{~g} \mathrm{~L}^{-1}$ using the expected glucose yield and a measured dilution factor, and $10 \mu \mathrm{L}$ of this diluted solution was transferred into a microcentrifuge tube containing $1.5 \mathrm{~mL}$ glucose HK reagent, which contains $1.5 \mathrm{mM}$ NAD, $1.0 \mathrm{mM}$ ATP, 1.0 unit $\mathrm{mL}^{-1}$ of hexokinase, and 1.0 unit $\mathrm{mL}^{-1}$ of glucose-6-phosphate dehydrogenase with sodium benzoate and potassium sorbate as preservatives Tubes were mixed by inversion, followed by incubation at room temperature for exactly 20 minutes before UV-Vis spectra acquisition (Agilent 8453). The absorbance of standard solutions at $340 \mathrm{~nm}$ was used to construct a calibration curve which was used to determine the concentration of glucose in the unknown samples, taking into account the appropriate dilution factor.

Quantification of ethanol using headspace gas chromatography mass spectrometry

After the fermentation reaction, the samples were centrifuged, filtered and stored in 20-mL vials on ice to minimize ethanol loss. To determine the ethanol concentration using headspace GC-MS, $3.0 \mathrm{~mL}$ of sample solution was transferred to a $20-\mathrm{mL}$ vial followed by the addition of a fixed amount ( 5 or $10 \mu \mathrm{L})$ of 2-propanol as an internal standard. These vials were placed in a $60{ }^{\circ} \mathrm{C}$ water bath for at least 10 minutes to increase the ethanol 
concentration in the headspace before the spectra of the samples were taken. A 0.5-mL headspace GC syringe was used to inject 10 to $50 \mu \mathrm{L}$ of the gas sample into the GC-MS for ethanol analysis. The GC acquisition method and calibration curve were established using standard ethanol solutions prepared in the same matrix. The initial temperature was set at 35 ${ }^{\circ} \mathrm{C}$ for 3 minutes and increased to $100{ }^{\circ} \mathrm{C}$ at a rate of $60{ }^{\circ} \mathrm{C} / \mathrm{min}$. The temperature of the injection port was $150{ }^{\circ} \mathrm{C}$. The ratio of the ethanol to 2-propanol peak area was utilized to account for differences in injection volume. A calibration curve was constructed to determine the amount of ethanol present in the reaction vials. 
Supplemental Table 1. Raman spectral assignments observed in this work and from a literature reference ${ }^{1}$ for an aqueous glucose solution.

\begin{tabular}{|c|c|c|}
\hline Observed $\left(\mathrm{cm}^{-1}\right)$ & Literature $^{1}\left(\mathrm{~cm}^{-1}\right)$ & Assignment $^{1}$ \\
\hline 427 & 436 & \multirow{2}{*}{$\delta(\mathrm{C}-\mathrm{C}-\mathrm{O}), \delta(\mathrm{C}-\mathrm{C}-\mathrm{C})$} \\
\hline 451 & 451 & \\
\hline 515 & 526 & $\delta(\mathrm{C} 2-\mathrm{C} 1-\mathrm{O} 1)$ \\
\hline 845 & 848 & \multirow{2}{*}{$\begin{array}{l}v(\mathrm{C}-\mathrm{C}), v(\mathrm{C}-\mathrm{O}), \delta(\mathrm{C}-\mathrm{C}-\mathrm{H}) \\
\delta(\mathrm{C}-\mathrm{C}-\mathrm{O}) \alpha \text {-glucose }\end{array}$} \\
\hline 915 & 916 & \\
\hline 1041 & 1041 & $v(\mathrm{C} 1-\mathrm{OH}) \alpha$-glucose \\
\hline 1064 & 1059 & $v(\mathrm{C} 1-\mathrm{OH}) \beta$-glucose \\
\hline 1125 & 1126 & $v(\mathrm{C}-\mathrm{C}), v(\mathrm{C}-\mathrm{O})$ \\
\hline 1262 & 1264 & $\delta(\mathrm{O}-\mathrm{C}-\mathrm{H}), \delta(\mathrm{C}-\mathrm{O}-\mathrm{H}), \delta(\mathrm{C}-\mathrm{C}-\mathrm{H})$ \\
\hline 1367 & 1365 & $\delta(\mathrm{C}-\mathrm{C}-\mathrm{H})$ \\
\hline 1460 & 1462 & $\mathrm{CH}_{2}$ vibration \\
\hline \multicolumn{3}{|c|}{$\begin{array}{l}\text { 1. Söderholm, S. et al. "Raman Spectra of Fructose and Glucose in th } \\
\text { Amorphous and Crystalline States" J. Raman Spectrosc. 1999, 30, } 1009 \\
1018 .\end{array}$} \\
\hline
\end{tabular}




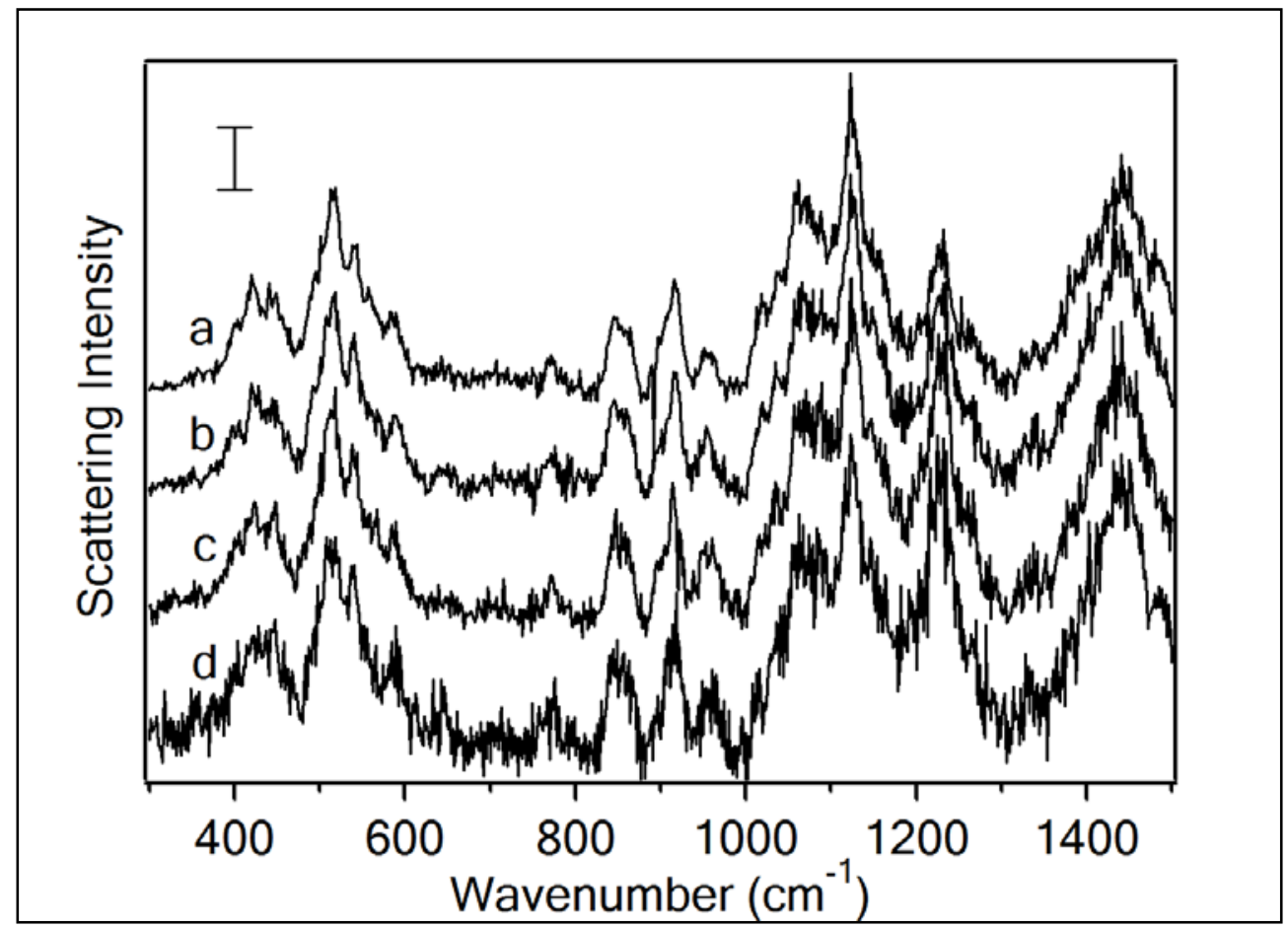

Supplemental Figure 1. Optimization of Raman spectroscopy acquisition time (a) 20 s; (b) $15 \mathrm{~s}$; (c) $10 \mathrm{~s}$; (d) $5 \mathrm{~s}$ for a $50 \mathrm{~g} \mathrm{~L}^{-1}$ glucose solution in acetate buffer. For each spectrum, 20 accumulations were collected. The scale bar represents a Raman scattering intensity of (a) 400; (b) 300; (c) 200; and (d) 100 counts. Spectra are offset for clarity. Peak assignments are shown in Supplemental Table 1. 


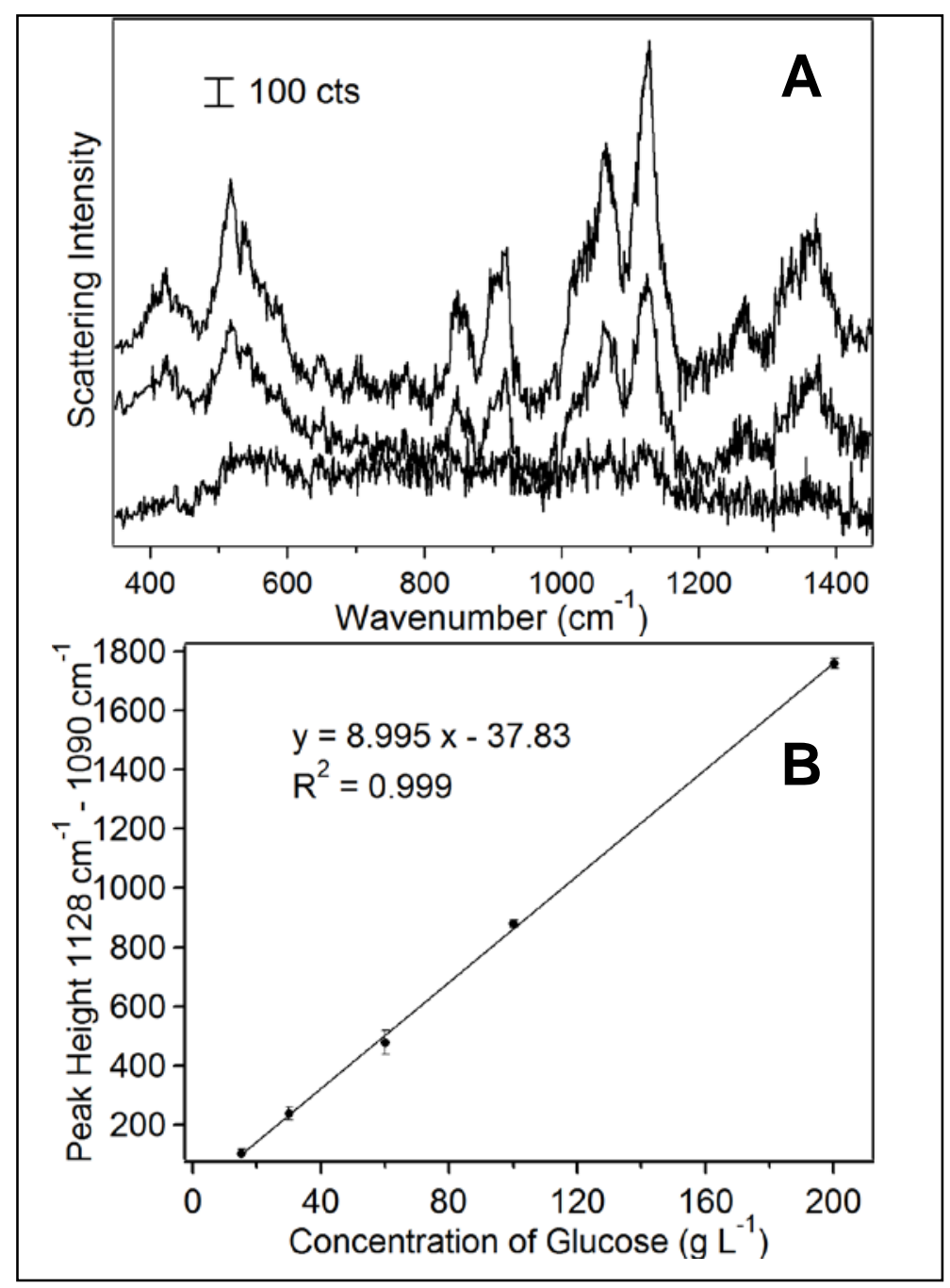

Supplemental Figure 2. (A) Spectra of (top) 100; (middle) 60; and (bottom) $15 \mathrm{~g} \mathrm{~L}^{-1}$ glucose solutions prepared in hydrolysis liquor. For all spectra, collection parameters are $10 \mathrm{~s}$ acquisition and 20 accumulations. Spectra are offset for clarity. (B) Glucose calibration curve by Raman spectroscopy. Error bars represent standard deviations from three replicate experiments. 


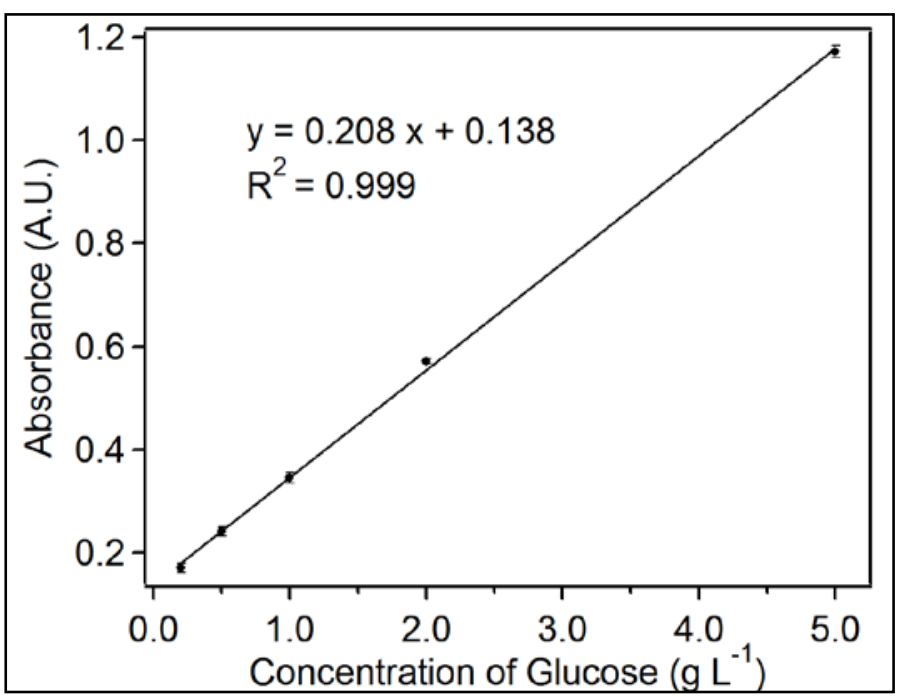

Supplemental Figure 3. Glucose calibration curve with standard glucose solutions prepared in hydrolysis liquor and measured using a glucose HK assay with UV-Vis spectrophotometry readout at $340 \mathrm{~nm}$. The hydrolysate samples ranged from 40 to $60 \mathrm{~g} \mathrm{~L}^{-1}$, and were diluted to the concentration range shown on the $\mathrm{x}$-axis prior to taking the $\mathrm{UV}-\mathrm{Vis}$ measurement. 


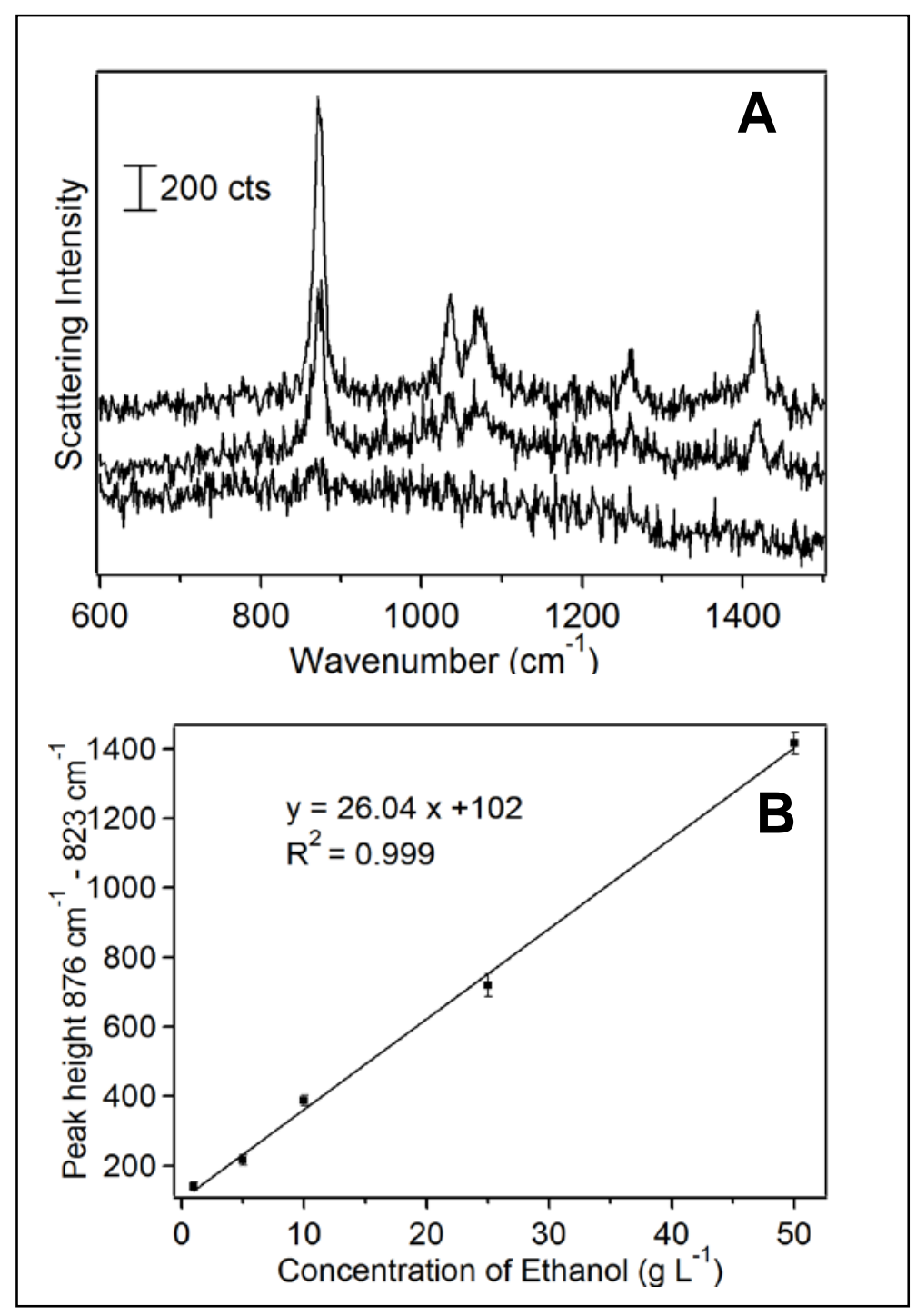

Supplemental Figure 4. (A) Raman spectra of (top) 50, (middle) 25, and (bottom) $5 \mathrm{~g} \mathrm{~L}^{-1}$ standard ethanol solutions prepared in fermentation liquor. All spectra are acquired for $10 \mathrm{~s}$ with 10 accumulations. Spectra are offset for clarity. The peak at $873 \mathrm{~cm}^{-1}$ is assigned to the symmetric C-C stretching mode of ethanol. (B) Ethanol calibration curve measured by Raman spectroscopy. Plotted is the peak intensity at $876 \mathrm{~cm}^{-1}$ minus the intensity at $823 \mathrm{~cm}^{-1}$ for each standard. Error bars represent standard deviations from three replicate experiments. 


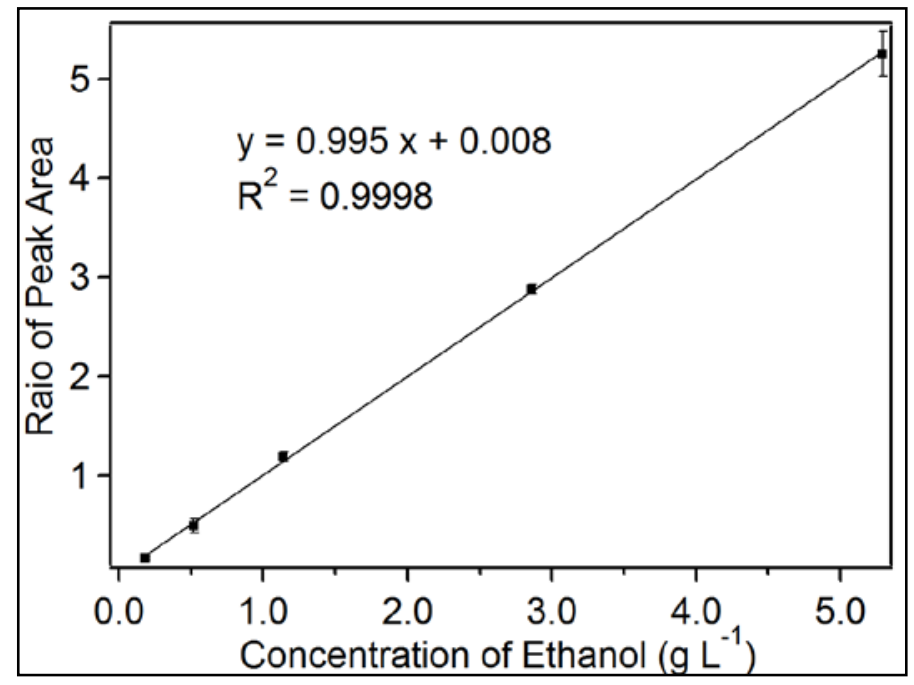

Supplemental Figure 5. Calibration curve for standard ethanol solutions prepared in fermentation liquor and measured using headspace gas chromatography-mass spectrometry. The $y$-axis is the ratio of the ethanol to internal standard (2-propanol) integrated peak area. 


\title{
CHPATER 3: RAMAN SPECTROSCOPY MEASUREMENTS OF
}

\author{
SACCHARIDES IN HYDROLYSATE USING DIFFERENT \\ BIOMASS PRETREATMENT PROTOCOLS AND ENZYME \\ COMPOSITIONS
}

\author{
Chien-Ju Shih ${ }^{1,2}$ and Emily A. Smith $*^{1,2}$
}

A manuscript submitted to Bioresource Technology

\begin{abstract}
Ames Laboratory, U.S. Department of Energy, Ames, Iowa 50011-3111, U.S.A.
Department of Chemistry, Iowa State University, Ames, Iowa 50011-3111, U.S.A.
\end{abstract}

\begin{abstract}
The effect of biomass pretreatment on the quantitative measurement of saccharides in hydrolysate by Raman spectroscopy is evaluated. Dilute sulfuric-acid pretreatment generated the highest glucose detection limit in hydrolysate $\left(20 \mathrm{mg} \mathrm{mL}^{-1}\right)$, and ammonia pretreatment generated a better detection limit $\left(5 \mathrm{mg} \mathrm{mL}^{-1}\right)$. Water and ethanol extractives produced the spectral background that limited glucose detection in dilute acid hydrolysate. Additionally, a multipeak fitting model is presented to simultaneously measure glucose and xylose
\end{abstract}

1. Ames Laboratory, U.S. Department of Energy, Ames, Iowa 50011-3111, U.S.A.

2. Department of Chemistry, Iowa State University, Ames, Iowa 50011-3111, U.S.A.

*Corresponding author 
concentration in the hydrolysate by Raman spectroscopy. This model yielded relative standard errors between 1.3 to $5.0 \%$ for most samples. When only cellulase was present, cellulose and xylan hydrolysis efficiencies were measured by Raman spectroscopy to be $66 \pm$ $4 \%$ and $28 \pm 1 \%$, respectively. When both cellulase and hemicellulase were present, xylan hydrolysis efficiencies increased to $71.4 \pm 0.8 \%$. The results were confirmed with complementary enzymatic or colorimetric assays.

Keywords: Raman Spectroscopy, Biomass Pretreatment, Glucose, Xylose, Enzymatic Hydrolysis, Spectral Multipeak Fitting

\section{Introduction}

Abundant lignocellulosic biomass, including corn stover, perennial grass, bagasse, wheat/rice straw and forestry/industry residue can be converted to portable biorenewable fuels in a multi-step process. In contrast to grain-based ethanol, lignocellulosic feedstocks do not compete with food production (Knauf and Moniruzzaman, 2004). Plant cell walls primarily contain a phenolic polymer, lignin, and carbohydrate polymers, hemicellulose and cellulose (Koshijima and Watanabe, 2003). In lignocellulosic feedstocks, it is the carbohydrate polymers in the plant cell wall that can be chemically or biologically hydrolyzed to monosaccharides and then fermented to produce bioethanol. Cellulose is a linear polymer of $\beta$-1,4-linked glucose units that is a key structural component in plants and can be hydrolyzed by cellulases. Hemicellulose is a heteropolysaccharide composed of various carbohydrate monomers with different linkages and substitutions on the primary branch (Pandey and Editor, 2009), and it can be hydrolyzed by hemicellulases. By dry mass, 
corn stover is about $36 \%$ cellulose and $30 \%$ hemicelluloses. The most abundant hemicellulose in corn stover is xylan (72\% of the total hemicelluloses) (U.S. DOE, 2004), which has a backbone of $\beta$-1,4-linked xylopyranose units. Lignin is a three-dimensional polymer consisting of syringyl-, guaiacyl-, and p-hydroxy-phenylpropanoid units (Ko et al., 2008). Since lignin in the cell wall interferes with the enzymatic hydrolysis of the sugar polymers, a critical biomass pretreatment step is required to disrupt the close association of the cell wall constituents for efficient enzymatic hydrolysis (Méchin et al., 2005).

Many protocols for biomass pretreatment have been developed, and each protocol is associated with advantages and disadvantages. Dilute acid pretreatment breaks down the cell wall structure and exposes the cellulose for increased enzyme accessibility (Mosier et al., 2005). Approximately $70 \%$ of the lignin, $5 \%$ of the hemicellulose, and $95 \%$ of the cellulose remains after dilute acid pretreatment of corn stover (Kim et al., 2003). In contrast, ammonia pretreatment breaks down the cell wall constituents and extracts a portion of the lignin from the cell wall (Kim and Lee, 2007). Approximately $15 \%$ of the lignin, $40 \%$ of the hemicellulose, and $90 \%$ of the cellulose remain after ammonia pretreatment of corn stover (Kim et al., 2003). On-going efforts aim to correlate efficient conversion methods with specific plant materials for the highest yield of fermentable sugars.

To date, many analytical methods have been developed to analyze monosaccharides in hydrolysate including high performance liquid chromatography (HPLC) (Öhgren et al., 2007; Sluiter et al., 2006) (Agblevor et al., 2007; Lloyd and Wyman, 2005; Raymond and Ehrman, 1996), ultraviolet (UV)-visible spectrophotometry (Cara et al., 2008), capillary electrophoresis (Huber et al., 1994), gas chromatography/mass spectrometry (Kamm et al., 2006) and electrochemical methods (Tatsumi and Katano, 2004). Compared to the analytical 
techniques mentioned above, vibrational spectroscopy (i.e. Raman and infrared spectroscopy) provides intrinsic chemical content information and multiple analytes can be measured simultaneously and non-invasively. In contrast to infrared methods, Raman spectroscopy has advantage for the analysis of aqueous samples due to water having a low Raman scattering efficiency in the region of 0 to $3000 \mathrm{~cm}^{-1}$ (Brooker et al., 1989; Zhang et al., 2005). Compared to IR spectra, Raman spectra generally have narrower peak widths for aqueous samples, cover a wide spectral range, and are better suited for quantitative measurements.

A previous study showed that the method used to treat biomass prior to enzymatic hydrolysis affected the Raman spectral background of the hydrolysate, and subsequently the quantitation of glucose in the hydrolysis broth (Shih and Smith, 2009). Dilute sulfuric acid pretreatment of biomass prohibited measurements of glucose at concentrations generated in typical hydrolysis reaction. On the other hand, ammonia pretreatment of biomass enabled glucose quantitation at concentration down to $8 \mathrm{mg} \mathrm{mL}^{-1}$. It was hypothesized that the differing abilities to measure glucose were due to the amount of residual lignin that remained in the pretreated biomass. The background may also results from intrinsic compounds that are extracted to different extents in the dilute acid and ammonia treated biomass. Additionally, small molecules (e.g., furfural) may be generated in the pretreatment step that cause spectral interference in subsequent analysis steps (Mosier et al., 2005). To date, dilute acid and ammonia pretreatment are among the most common chemical pretreatment methods being studied (Chen et al., 2009; Eggeman and Elander, 2005; Kumar and Wyman Charles, 2009; Wyman et al., 2005). Understanding the sources of the spectral background after different biomass pretreatments could expand the utility of Raman spectroscopy for biofuel research, by lowering the saccharide detection limit in hydrolysate. 
Herein, the $785 \mathrm{~nm}$ excitation Raman spectroscopy detection limit of glucose in corn stover hydrolysate for different pretreatment protocols is reported. To date, attention has been paid to the amount of glucose generated in the hydrolysate. Less attention has been focused on the amount of xylose produced. This is due, in part, to the fact that the commercial hydrolysis enzymes have high cellulase activity and low hemicellulase activity. In order to significantly increase total sugar yields, commercial enzyme mixtures containing or supplemented with hemicellulases are being used. Raman spectroscopy can be used to analyze multiple analytes in the matrix. A multipeak fitting model was developed to simultaneously measure glucose and xylose in the complex hydrolysis matrix.

\section{Materials and methods}

\subsection{Materials}

All chemicals were reagent grade. Sodium acetate trihydrate, glacial acetic acid, sulfuric acid, ethanol and ammonia were purchased from Fisher Scientific (Pittsburgh, PA). Cycloheximide, tetracycline (Fluka), D-glucose, microcrystalline cellulose, xylan, phloroglucinol and glucose HK reagent were purchased from Sigma-Aldrich (St. Louis, MO). Accellerase $1500^{\circledR}$ enzyme and Accellerase $\mathrm{XY}{ }^{\circledR}$ hemicellulase enzyme complex were provided by Genencor (Rochester, NY). All solutions were prepared using ultrapure deionized (DI) water (18.2 $\mathrm{M} \Omega \mathrm{cm}^{-1}$ ) from an Easypure II water system (Barnstead Int.).

\subsection{Soxhlet extraction}

The two-step Soxhlet extraction of corn stover using water followed by ethanol was performed as outlined in a National Renewable Energy Laboratory protocol (Sluiter et al., 2005). Approximately 5 g dry corn stover was loaded in a cotton cellulose thimble and 
placed in a glass Soxhlet extraction tube. A round-bottom flask containing $250 \mathrm{~mL}$ water was connected to the extraction tube and heated to reflux for 24 hours. This was repeated with $250 \mathrm{~mL}$ ethanol for 24 hours.

\subsection{Enzymatic hydrolysis reactions}

Biomass pretreatment by dilute acid or ammonia was performed as previously outlined (Shih and Smith, 2009). Enzymatic hydrolysis reactions were carried out in $0.1 \mathrm{M}$ acetate buffer, $\mathrm{pH} 4.8$ with addition of antibiotics. Approximately $7 \mathrm{~g}$ of wet pretreated corn stover (corresponding to $1 \mathrm{~g}$ of dry biomass) was placed in a $40-\mathrm{mL}$ amber glass vial followed by the addition of $0.80 \mathrm{~mL}$ Accellerase $1500^{\mathrm{TM}}$ enzyme, and/or $0.2 \mathrm{~mL}$ Accellerase $\mathrm{XY}$ enzyme, $200 \mu \mathrm{L}$ cycloheximide, $30 \mu \mathrm{L}$ tetracycline, and acetate buffer to bring the total volume to $13 \mathrm{~mL}$. These vials were placed in an incubated shaker at $50{ }^{\circ} \mathrm{C}, 200 \mathrm{rpm}$ for 48 hours. The samples were heated to $60^{\circ} \mathrm{C}$ for 10 minutes to terminate the enzymatic reaction at the end of the incubation period. Samples were then centrifuged at approximately $6200 \mathrm{~g}$ for 30 minutes and the supernatants were filtered using a 0.22 micron filter.

\subsection{Determination of detection limits}

The equivalent of 1 gram of dry biomass pretreated with sulfuric acid (SA), ammonia (AM), Soxhlet extraction (E), or in combination (E-SA, E-AM) was used in a simulated hydrolysis experiment to create representative matrices for each pretreatment method. The simulated hydrolysis was performed as described in section 2.3, except the enzyme was denatured prior to adding it to the reaction vials. After 48 hours, the mixtures were centrifuged at approximately $6200 \mathrm{~g}$ for 30 minutes and the supernatants were filtered using a 0.22 micron filter. The broths were subsequently used to prepare standard solutions with known glucose concentrations, and were measured by Raman spectroscopy. 
The $1123 \mathrm{~cm}^{-1}$ peak intensity for the standard solutions was used to construct the Raman spectroscopy calibration curves, which were fit using partial least square regression. All calibration curves in this study had coefficients of determination $\left(\mathrm{R}^{2}\right)$ greater than 0.98 , indicating that the concentration can be predicted by the model. Detection limits were calculated by analyzing 7 replicate samples with concentrations near the detection limit and 1 order of magnitude higher, and computing 3 times the standard deviation of the replicate measurements divided by the slope of the calibration curve (Harris, 2007).

\subsection{Quantitative analysis of glucose and xylose}

Standard solutions of xylose (10 to $100 \mathrm{mg} \mathrm{mL}^{-1}$ ) and glucose (20 to $120 \mathrm{mg} \mathrm{mL}^{-1}$ ) in hydrolysis broth were prepared using serial dilution and were measured by Raman spectroscopy in triplicate. For each concentration, a multipeak fitting algorithm was used to identify the location, intensity, and width of the peaks in the spectral region between 350 to $650 \mathrm{~cm}^{-1}$. Eight peaks were identified in this spectral region for glucose and six were identified for xylose. The intensity, as determined by deconvolution of the peaks at 519 $\mathrm{cm}^{-1}$ for glucose and $536 \mathrm{~cm}^{-1}$ for xylose, was used to generate a calibration curve.

Mixtures of glucose and xylose were prepared in 2:1, 3:1, and 4:1 ratios at two glucose concentrations (30 and $60 \mathrm{mg} \mathrm{mL}^{-1}$ ) to test the calibration curves. These ratios represent the approximate amount of cellulose and hemicellulose in untreated corn stover (2:1), and composition after ammonia pretreatment (4:1). Raman spectra were collected for each mixture, and the spectra were fit using the multipeak fitting parameters. Specifically, fourteen peaks were used in the fit, and the Raman shift and width of each of the 14 peaks were set to the value obtained from the single component spectra. The peak intensities that minimized the residual between the experimental data and the fit were identified. The best fit 
peak intensities were used to determine the glucose and xylose concentration using the developed calibration curves. This same method was used to measure the glucose and xylose concentration resulting from the cellulase or cellulase/xylanase hydrolysis of ammoniapretreated corn stover.

\subsection{Raman spectroscopy measurements}

A home-built Raman microscope consisting of a 785-nm diode laser, inverted optical microscope, spectrometer and an IR-enhanced CCD camera was used for all measurements as previously described (Shih and Smith, 2009). An $800 \mu \mathrm{m}$ pinhole replaced the $100 \mu \mathrm{m}$ pinhole used in previous studies. The Raman scatter was collected at room temperature using 10 second integrations with 20 accumulations for all measurements. The laser power for all measurements was approximately $130 \mathrm{~mW}$ at the sample. Near IR (e.g., $785 \mathrm{~nm}$ ) excitation is critical to minimize sample fluorescence, which renders the Raman spectrum of hydrolysate undetectable.

\subsection{UV-visible spectrophotometry measurements}

Glucose HK assays were used as confirmatory tests of glucose concentration, (Bondar and Mead, 1974) and phloroglucinol-based colorimetric assays (Eberts et al., 1979; Johnson et al., 1984) were used to confirm xylose concentration. A calibration curve using standard solutions was generated for each assay. A best fit line was generated by partial least square regression. All samples were diluted to the linear range of the calibration curve before measurement. Each sample was measured in triplicate and the average value and 95\% confidence intervals were reported. 


\subsection{Spectral analysis and statistical analysis}

Spectra were collected using Winspec32 (Roper Scientific, Germany) and were processed using GRAMS and Igor Pro 6.1 (WaveMetrics. Inc.) to perform background subtraction and multipeak fitting, respectively. All spectra were subtracted by a blank consisting of the sample matrix without analyte(s) and were measured in triplicate, unless otherwise noted above.

\section{Results and discussion}

3.1 The effect of biomass pretreatment on Raman spectral background for quantitative saccharide measurements

High spectral background values limit the quantitative measurement of glucose in hydrolysate. The source of the spectral background in Raman measurements of hydrolysate was systematically evaluated using several biomass pretreatment protocols and subsequently, the glucose detection limit was measured by adding glucose in simulated hydrolysis broth (Figure 1). Specifically, biomass pretreated with one of six protocols was used in a simulated hydrolysis reaction that contained inactive enzyme, but was otherwise carried out using standard hydrolysis conditions. Soluble species present in the biomass after pretreatment will leach into the hydrolysis broth. Insoluble components do not interfere with the Raman spectral measurements since the samples are centrifuged and filtered prior to analysis. The supernatant of hydrolysis broth was then used to prepare standard glucose samples, which were analyzed by Raman spectroscopy. A calibration curve was constructed and the glucose detection limit was determined. 
The pretreatment methods that were used in this study were dilute sulfuric acid (SA) and ammonia (AM). In addition, a Soxhlet extraction method reported by NREL was utilized to remove extractives in the biomass (Sluiter et al., 2005), which represent approximately 3 to $6 \%$ of the total dry mass of corn stover. The Soxhlet extraction was chosen to evaluate whether small molecule extractives contribute to the spectral background measured by Raman spectroscopy. Water extraction was used to remove inorganic materials, nonstructural sugars, and nitrogenous material. In the second step, ethanol was used to remove chlorophyll, waxes, or other minor components (Sluiter et al., 2005). Extraction is not known to disrupt cell wall components or increase glucose yield, and is not commonly used as a pretreatment step in the bioethanol industry. Extraction was used alone (E), or in combination with dilute acid (E-SA) or ammonia pretreatment (E-AM). Comparisons were also performed with untreated biomass.

The limit of glucose detection can be determined from the calibration curve constructed for each hydrolysis broth using the intensity of the $1122 \mathrm{~cm}^{-1}$ spectral peak (Table 1). This peak represents C-C or C-O stretching and is the most intense glucose peak. The calibration curves and limits of detection will vary depending on the chosen spectral peak. Since the relative standard deviation near the lower limit of detection is around 33\%, only one significant figure is reported.

Dilute sulfuric acid pretreated and untreated biomass hydrolysis broths have the poorest glucose detection limit $\left(20 \mathrm{mg} \mathrm{mL}^{-1}\right)$ among the six pretreatment methods studied. The quantification limit is usually reported to be 3.3 times of the lower limit of detection. Expected glucose yields from the hydrolysis reaction are usually between 30 to $70 \mathrm{mg} \mathrm{mL}^{-1}$ using our experimental set up. Glucose can be quantified precisely in untreated or dilute 
sulfuric acid hydrolysate using Raman spectroscopy when hydrolysis efficiencies exceed $79 \%$ or $100 \%$ respectively. Since the detection limit is the same for both sulfuric acid and untreated biomass (i.e. the spectral background and noise were similar), this suggests that the source of the spectral background is intrinsic to the biomass and not generated during the acid pretreatment.

Extracting water and ethanol soluble compounds from biomass (E), without any further pretreatment, lowers the glucose detection limit to $4 \mathrm{mg} \mathrm{mL}^{-1}$, indicating that extractives contribute to the spectral background observed in the untreated biomass hydrolysis liquor. This is further supported by the measurement for extracted plus dilute acid pretreated biomass (E-SA). In this case, the detection limit is approximately the same (5 mg $\mathrm{mL}^{-1}$ ) as that for extracted biomass alone, and supports the assumption that the spectral background measured in dilute acid hydrolysis liquor is from extractable compounds.

Ammonia pretreatment produces a detection limit $\left(4 \mathrm{mg} \mathrm{mL}^{-1}\right)$ that is the same as that measured for extracted biomass. This is better than the previously reported detection limit (8 $\mathrm{mg} \mathrm{mL} \mathrm{m}^{-1}$ ) due to improved detection optics on the Raman spectrometer (Shih and Smith, 2009). A neutralization and rinse step after ammonia pretreatment was found to be crucial for lowering the detection limit, which was $16 \mathrm{mg} \mathrm{mL}^{-1}$ when this step was omitted. The detection limit for biomass that is extracted and then pretreated with ammonia $\left(5 \mathrm{mg} \mathrm{mL}^{-1}\right)$ is also approximately the same as the value for the ammonia pretreated biomass. This suggests that the ammonia pretreatment extracts the soluble products that contribute to the background observed in the untreated biomass.

The reported detection limits do not necessarily correlate directly with measurable hydrolysis efficiencies from subsequent hydrolysis reactions. As mentioned above, the 
pretreatment methods extract small, varying amounts of cellulose from the cell wall, and the amount of cellulose at the start of the hydrolysis reaction varies. Assuming the amount of cellulose in extracted and untreated biomass does not change, and using average values for the amount of cellulose removed in acid and ammonia pretreated biomass, the lower limits of quantification have been correlated with the minimum hydrolysis efficiencies that can be measured by Raman spectroscopy using $785 \mathrm{~nm}$ excitation (Table 1). A range of hydrolysis efficiencies for different biomass types and pretreatment steps is between $56 \%$ and $92 \%$ (Kim et al., 2003; Lloyd and Wyman, 2005). Thus, Raman spectroscopy would be suitable for glucose quantitation in E, E-SA, E-AM, AM hydrolysates. The hydrolysis efficiency is likely to be lower than $40 \%$ for extracted biomass since no report has shown it disrupts the cell wall polymer bonding, as is required for efficient enzymatic hydrolysis. Raman spectroscopy may have limited utility in this case (E).

\subsection{Simultaneous determination of glucose and xylose in hydrolysis liquor}

Deconvoluting overlapping spectral peaks can be used to identify and quantify molecules that do not exhibit unique spectral features. Xylan is approximately $72 \%$ of the total hemicellulose in corn stover, and its primary hydrolysis product is xylose. A multipeak fitting model was developed using spectra of glucose and xylose standard solutions prepared in hydrolysis broth at concentrations ranging from 10 to $100 \mathrm{mg} \mathrm{mL}^{-1}$. The 350 to $650 \mathrm{~cm}^{-1}$ spectral region was chosen for deconvolution because the most intense peaks for each sugar had distinguishable peak maxima in this region. In order to perform the peak deconvolution, baseline correction is needed. This is most critical at low saccharide concentrations (below $30 \mathrm{mg} \mathrm{mL}^{-1}$ ). A linear baseline correction was performed for the 350 to $650 \mathrm{~cm}^{-1}$ spectral region. The linear correction has two numbers that need to be determined, the intercept and 
the slope. These parameters were set by ensuring the baseline correcting line passed through the data points at 378 and $628 \mathrm{~cm}^{-1}$.

The spectra (solid lines), multipeak fits (thin lines), and residuals for $120 \mathrm{mg} \mathrm{ml}^{-1}$ glucose and $100 \mathrm{mg} \mathrm{ml}^{-1}$ xylose solution are shown in Figure 2A and B, respectively. After deconvoluting the spectra for each sugar at all concentrations, the Raman shifts and the peak widths were averaged. These parameters are shown in Table 2. The Raman shift varied by no more than $1.1 \mathrm{~cm}^{-1}$, and the peak width varied by no more than $1.2 \mathrm{~cm}^{-1}$ across all concentrations used to develop the average.

A calibration curve was constructed for glucose $\left(519 \mathrm{~cm}^{-1}\right)$ and xylose $\left(536 \mathrm{~cm}^{-1}\right)$ using the peak intensity, as determined by spectral deconvolution. This calibration curve was employed to determine the glucose and xylose concentration in mixtures with known saccahride concentrations. The xylose detection limit in ammonia pretreated biomass hydrolysate was $1 \mathrm{mg} \mathrm{mL}^{-1}$.

The spectrum of a $60 \mathrm{mg} \mathrm{mL}^{-1}$ glucose and $50 \mathrm{mg} \mathrm{mL}^{-1}$ xylose mixture in the hydrolysis broth is shown in figure 2C. A total of 14 peaks were used in the fit (8 peaks from glucose and 6 peaks from xylose). Using the parameters listed in Table 2, a good fit is achieved between the data and the model, as shown by the residual. After baseline correction and peak deconvolution, the peak intensity and calibration curves were used to obtain the concentration of glucose and xylose. These were determined to be $64 \pm 5$ and $54 \pm 5 \mathrm{mg} \mathrm{mL}^{-1}$, respectively.

The multipeak algorithm was then tested with mixtures of glucose and xylose at three ratios and two concentrations. These concentrations and ratios were selected based on expected yields in actual hydrolysis reactions where the cellulase is supplemented with 
hemicellulase. Using the procedure described above, the concentration of each sugar was determined and is reported in Table 3. With one exception, the results are in good agreement at the $95 \%$ confidence level. The relative standard error is between $1.3 \%$ to $5.0 \%$ at the higher glucose concentration $\left(60 \mathrm{mg} \mathrm{mL}^{-1}\right)$ and $2.5 \%$ to $17 \%$ at a lower glucose concentration (30 $\mathrm{mg} \mathrm{mL}^{-1}$ ). The $17 \%$ relative standard deviation was observed at a 4:1 ratio. This suggested that this methodology has limitation on determining low xylose concentrations when glucose is 4 times more abundant than xylose.

The multipeak fit was tested for its ability to measure glucose and xylose in corn stover hydrolysis reactions. The ammonia-pretreated biomass was chosen for this experiment. Three enzyme combinations were used: Accellerase 1500 (cellulase), Accellerase 1500/XY (hemicellulase), and XY. The accessory enzyme XY was reported to have high activity towards xylan, the main component of corn stover hemicellulose. A sample containing all reaction components except enzyme was used as a blank, and its spectrum was subtracted from the other three spectra.

The spectra of hydrolysis broth generated from the Accellerase 1500 enzyme and the Accllerase 1500/XY enzyme mixture was deconvoluted as described above. The hydrolysis efficiencies are shown in Table 4. The sample containing only enzyme XY did not show interpretable peaks, possibly because the xylan was not accessible to the xylanase.

The amount of glucose measured by Raman spectroscopy did not significantly differ when only cellulase was present (66 $\pm 9 \%$ hydrolysis efficiency) or when both cellulase and hemicellulase were present (64 $\pm 8 \%$ hydrolysis efficiency) in the reaction. The Raman spectroscopy results were confirmed using glucose-specific HK assays. The results obtained by UV- visible spectrophotometry agreed with the Raman spectroscopy results within the 
95\% confidence interval. A small amount of other monosaccharides or soluble oligosaccharides (e.g., cellobiose) may contribute to the Raman signal; however, the similarities of the Raman and glucose specific HK assay results suggested that this contribution was negligible or not present. In the literature, it has been reported that supplementation of cellulase with xylanase could improve glucose yield. (Kumar and Wyman, 2009) The increase in glucose production depended on the concentration of the enzyme, and the pretreatment method. The ammonia recycled percolation pretreatment method produced the lowest increase in glucose with hemicellulase supplementation, and this is closest to the pretreatment method used in these studies. This is a possible explanation for why an increase in glucose was not measured by Raman spectroscopy or glucose HK assays when the cellulase was supplemented with hemicellulase.

As measured by Raman spectroscopy, the addition of hemicellulase to the hydrolysis reaction increased xylose yield by $\sim 2.5$ times compared to the reaction with only cellulase present. This increase is consistent with literature reports (Kumar and Wyman, 2009). A phloroglucinol colorimetric assay was used to confirm the concentration of xylose. The advantage of this assay (Johnson et al., 1984) is that there is little interference from glucose or proteins (e.g., cellulase and hemicellulase). The UV-visible spectrophotometry results agree with the Raman spectroscopic data within the 95\% confidence interval, and suggest that Raman spectroscopy can be used to simultaneously measure the yield of two monosaccharides in biomass hydrolysate. 


\section{Conclusions}

Raman spectroscopy requires minimal sample preparation and analysis time; it is non-invasive, suitable for complex sample matrices, can be automated, and can be a useful analytical tool in the production of bioethanol. Both ammonia and dilute acid pretreated biomass hydrolysate can be analyzed by Raman spectroscopy, but dilute acid pretreated biomass first must be extracted with water and ethanol. Glucose and xylose yield in corn stover hydrolysate can be measured accurately by Raman spectroscopy using a multipeak fitting model. Similar methods could be applied to measure total sugar yields from other feedstocks with appropriate calibration, or for real-time reaction monitoring.

\section{Acknowledgements}

Work at the Ames Laboratory was supported by the Department of Energy-Basic Energy Sciences under Contract No. DE-AC02-07CH11358. The authors are grateful to Dr. Kenneth J. Moore for providing corn stover and Genecor International for providing the Accellerase enzymes.

\section{References}

Agblevor, F.A., Hames, B.R., Schell, D., Chum, H.L., 2007. Analysis of biomass sugars using a novel HPLC method. Appl. Biochem. Biotechnol., 136, 309-326.

Bondar, R.J., Mead, D.C., 1974. Evaluation of glucose-6-phosphate dehydrogenase from Leuconostoc mesenteroides in the hexokinase method for determining glucose in serum. Clin. Chem., 20, 586-90.

Brooker, M.H., Hancock, G., Rice, B.C., Shapter, J., 1989. Raman frequency and intensity studies of liquid water (H2O, H218O and D2O). J. Raman Spectrosc., 20, 683-94.

Cara, C., Ruiz, E., Oliva, J.M., Sáez, F., Castro, E., 2008. Conversion of olive tree biomass into fermentable sugars by dilute acid pretreatment and enzymatic saccharification. Bioresour. Technol., 99, 1869-1876.

Chen, M., Zhao, J., Xia, L., 2009. Comparison of four different chemical pretreatments of corn stover for enhancing enzymatic digestibility. Biomass Bioenergy, 33, 1381-1385. 
Eberts, T.J., Sample, R.H.B., Glick, M.R., Ellis, G.H., 1979. A simplified, colorimetric micromethod for xylose in serum or urine, with phloroglucinol. Clin. Chem. (Winston-Salem, N. C.), 25, 1440-3.

Eggeman, T., Elander, R.T., 2005. Process and economic analysis of pretreatment technologies. Bioresour. Technol., 96, 2019-2025.

Harris, D.C., 2007. Quantitative Chemical Analysis. 7th ed. Craig Bleyer, New York.

Huber, C., Grill, E., Oefner, P., Bobleter, O., 1994. Capillary electrophoretic determination of the component monosaccharides in hemicelluloses. Fresenius' J. Anal. Chem., 348, 825-31.

Johnson, S.L., Bliss, M., Mayersohn, M., Conrad, K.A., 1984. Phloroglucinol-based colorimetry of xylose in plasma and urine compared with a specific gaschromatographic procedure. Clin. Chem. (Winston-Salem, N. C.), 30, 1571-4.

Kamm, B., Kamm, M., Schmidt, M., Starke, I., Kleinpeter, E., 2006. Chemical and biochemical generation of carbohydrates from lignocellulose-feedstock (Lupinus nootkatensis)-quantification of glucose. Chemosphere, 62, 97-105.

Kim, T.H., Kim, J.S., Sunwoo, C., Lee, Y.Y., 2003. Pretreatment of corn stover by aqueous ammonia. Bioresour. Technol., 90, 39-47.

Kim, T.H., Lee, Y.Y., 2007. Pretreatment of corn stover by soaking in aqueous ammonia at moderate temperatures. Appl. Biochem. Biotechnol., 137-140, 81-92.

Knauf, M., Moniruzzaman, M., 2004. Lignocellulosic biomass processing: a perspective. Int. Sugar J., 106, 147-150.

Ko, J.-J., Shimizu, Y., Ikeda, K., Kim, S.-K., Park, C.-H., Matsui, S., 2008. Biodegradation of high molecular weight lignin under sulfate reducing conditions: Lignin degradability and degradation by-products. Bioresour. Technol., 100, 1622-1627.

Koshijima, T., Watanabe, T., 2003. Association Between Lignin and Carbohydrates in Wood. Springer- Verlag Berlin Heidelberg, New York.

Kumar, R., Wyman, C.E., 2009. Effect of xylanase supplementation of cellulase on digestion of corn stover solids prepared by leading pretreatment technologies. Bioresour. Technol., 100, 4203-4213.

Kumar, R., Wyman Charles, E., 2009. Cellulase adsorption and relationship to features of corn stover solids produced by leading pretreatments. Biotechnol Bioeng., 103, 25267.

Lloyd, T.A., Wyman, C.E., 2005. Combined sugar yields for dilute sulfuric acid pretreatment of corn stover followed by enzymatic hydrolysis of the remaining solids. Bioresour. Technol., 96, 1967-1977.

Méchin, V., Argillier, O., Rocher, F., Hébert, Y., Mila, I., Pollet, B., Barriére, Y., Lapierre, C., 2005. In Search of a Maize Ideotype for Cell Wall Enzymatic Degradability Using Histological and Biochemical Lignin Characterization. J. Agric. Food Chem., 53, 5872-5881.

Mosier, N., Wyman, C., Dale, B., Elander, R., Lee, Y.Y., Holtzapple, M., Ladisch, M., 2005. Features of promising technologies for pretreatment of lignocellulosic biomass. Bioresour. Technol., 96, 673-686.

Öhgren, K., Vehmaanperä, J., Siika-Aho, M., Galbe, M., Viikari, L., Zacchi, G., 2007. High temperature enzymatic prehydrolysis prior to simultaneous saccharification and 
fermentation of steam pretreated corn stover for ethanol production. Enzyme Microb. Technol., 40, 607-613.

Pandey, A., Editor, 2009. Handbook of Plant-Based Biofuels.

Raymond, R., Ehrman, T., 1996. HPLC Analysis of Liquid Fractions of Process Samples for Monomeric Sugars and Cellobiose Laboratory Analytical Procedure (LAP-013). National Renewable Energy Laboratory, Golden, CO USA.

Shih, C.-J., Smith, E.A., 2009. Determination of glucose and ethanol after enzymatic hydrolysis and fermentation of biomass using Raman spectroscopy. Anal. Chim. Acta, 653, 200-206.

Sluiter, A., Hames, B., Ruiz, R., Scarlata, C., Sluiter, J., Templeton, D., 2006. Determination of Sugars, Byproducts, and Degradation Products in Liquid Fraction Process Samples. in: B.A.T. Team (Ed.) Laboratory Analytical Procedure (LAP). National Renewable Energy Laboratory, Golden, CO USA.

Sluiter, A., Ruizx, R., Scarlata, C., Sluiter, J., Templeton, D., 2005. Determination of Extractives in Biomass. NREL Laboratory Analytical Procedure (LAP).

Tatsumi, H., Katano, H., 2004. Kinetic analysis of enzymatic hydrolysis of raw starch by glucoamylase using an amperometric glucose sensor. Chem. Lett., 33, 692-693.

U.S. DOE, 2004. Biomass Feedstock Composition and Property Database. http://www.afdc.energy.gov/biomass/progs/search1.cgi.

Wyman, C.E., Dale, B.E., Elander, R.T., Holtzapple, M., Ladisch, M.R., Lee, Y.Y., 2005. Comparative sugar recovery data from laboratory scale application of leading pretreatment technologies to corn stover. Bioresour. Technol., 96, 2026-2032.

Zhang, X., Young, M.A., Lyandres, O., Van Duyne, R.P., 2005. Rapid detection of an anthrax biomarker by surface-enhanced Raman spectroscopy. J. Am. Chem. Soc., 127, 4484-4489. 
Table 1: Glucose Raman spectroscopy detection limit and associated minimum detectable hydrolysis efficiency for the biomass hydrolysate generated using six different pretreatment protocols.

\begin{tabular}{|l|c|c|}
\hline $\begin{array}{l}\text { Pretreatment } \\
\text { Protocol }\end{array}$ & $\begin{array}{l}\text { Lower limit of } \\
\text { Detection }\left(\mathbf{m g ~ m L}^{-1}\right)\end{array}$ & $\begin{array}{l}\text { Lowest Detectable } \\
\text { Hydrolysis Efficiency (\%) }\end{array}$ \\
\hline E & 4 & 19 \\
\hline E-SA & 5 & 26 \\
\hline E-AM & 5 & 24 \\
\hline AM & 4 & 18 \\
\hline SA & 20 & $>100$ \\
\hline Untreated & 20 & 79 \\
\hline
\end{tabular}

1 Accounting for cellulose loses during sulfuric acid (5\%) and ammonia (10\%) pretreatment. 
Table 2: Raman shifts and peak widths calculated from glucose and xylose standards that were used for the quantitative analysis of hydrolysis products.

\begin{tabular}{|c|c|c|c|}
\hline Peak & Raman Shift $\left(\mathrm{cm}^{-1}\right)$ & Width $\left(\mathrm{cm}^{-1}\right)$ & Analyte \\
\hline $\mathbf{0}$ & 408 & 16.5 & Glucose \\
\hline 1 & 417 & 22.7 & Xylose \\
\hline 2 & 423 & 7.59 & Glucose \\
\hline 3 & 448 & 20.9 & Glucose \\
\hline 4 & 471 & 16.2 & Xylose \\
\hline 5 & 500 & 18.6 & Glucose \\
\hline 6 & 508 & 10.6 & Xylose \\
\hline 7 & 519 & 10.7 & Glucose \\
\hline 8 & 536 & 13.6 & Xylose \\
\hline 9 & 540 & 9.89 & Glucose \\
\hline 10 & 558 & 15.8 & Xylose \\
\hline 11 & 562 & 14.5 & Glucose \\
\hline 12 & 589 & 13.8 & Glucose \\
\hline 13 & 602 & 14.3 & Xylose \\
\hline
\end{tabular}


Table 3: Glucose and xylose concentrations measured using multipeak fitting analyses on the Raman spectra of standard solutions prepared in hydrolysis broth.

\begin{tabular}{|l|l|l|}
\hline $\begin{array}{l}\text { Composition } \\
\text { eq=equivalents }\end{array}$ & Glucose $=60 \mathrm{mg} / \mathrm{mL}^{\mathbf{1}}$ & Glucose $=30 \mathrm{mg} / \mathrm{mL}^{\mathbf{1}}$ \\
\hline 2 eq glucose & $60 \pm 3$ & $29 \pm 3$ \\
\hline 1 eq xylose & $28 \pm 3$ & $15 \pm 1$ \\
\hline 3 eq glucose & $59 \pm 3$ & $28 \pm 3$ \\
\hline 1 eq xylose & $19 \pm 1$ & $9 \pm 3$ \\
\hline 4 eq glucose & $61 \pm 4$ & $7 \pm 3$ \\
\hline 1 eq xylose & $14 \pm 1$ & $32 \pm 2$ \\
\hline 1 All solutions & & \\
\hline
\end{tabular}

1 All solutions were measured in triplicate. Average $\pm 95 \%$ confidence interval. 
Table 4: Measured hydrolysis efficiencies for ammonia-pretreated biomass treated with cellulase (Accellerase 1500) or both cellulase and hemicellulase (Accellerase $1500+$ XY).

\begin{tabular}{|l|l|l|}
\hline Method/Analyte & $\begin{array}{l}\text { Accellerase 1500 } \\
\text { Hydrolysis Efficiency (\%) }\end{array}$ & $\begin{array}{l}\text { Accellerase 1500 + XY } \\
\text { Hydrolysis Efficiency (\%) }\end{array}$ \\
\hline Raman/glucose & $66 \pm 9$ & $64 \pm 8$ \\
\hline UV-Vis/glucose & $68 \pm 8$ & $62 \pm 21$ \\
\hline Raman/xylose & $28 \pm 3$ & $71 \pm 2$ \\
\hline UV-Vis/xylose & $26 \pm 3$ & $75 \pm 25$ \\
\hline
\end{tabular}

1 All solutions were measured in triplicate. Average $\pm 95 \%$ confidence interval. 


\section{Figure Captions}

Figure 1: Overview of the measurements performed to evaluate Raman spectroscopy as an analysis method for the enzymatic hydrolysis of biomass under different experimental conditions. The glucose detection limit was measured for 6 pretreatment protocols. A multipeak fitting algorithm was developed to quantify glucose and xylose from the hydrolysate of ammonia-pretreated biomass.

Figure 2: Multipeak fitting results for (A) $120 \mathrm{mg} \mathrm{mL}^{-1}$ glucose; (B) $100 \mathrm{mg} \mathrm{mL}^{-1}$ xylose; and (C) a $60 \mathrm{mg} \mathrm{mL}^{-1}$ glucose $/ 50 \mathrm{mg} \mathrm{mL}^{-1}$ xylose mixture in hydrolysis broth. The spectra are shown in the middle panel (thicker line) and the fit results are shown in the bottom panel (thinner line). The indicated peak numbers correspond to the peaks identified in Table 2. 
Figure 1

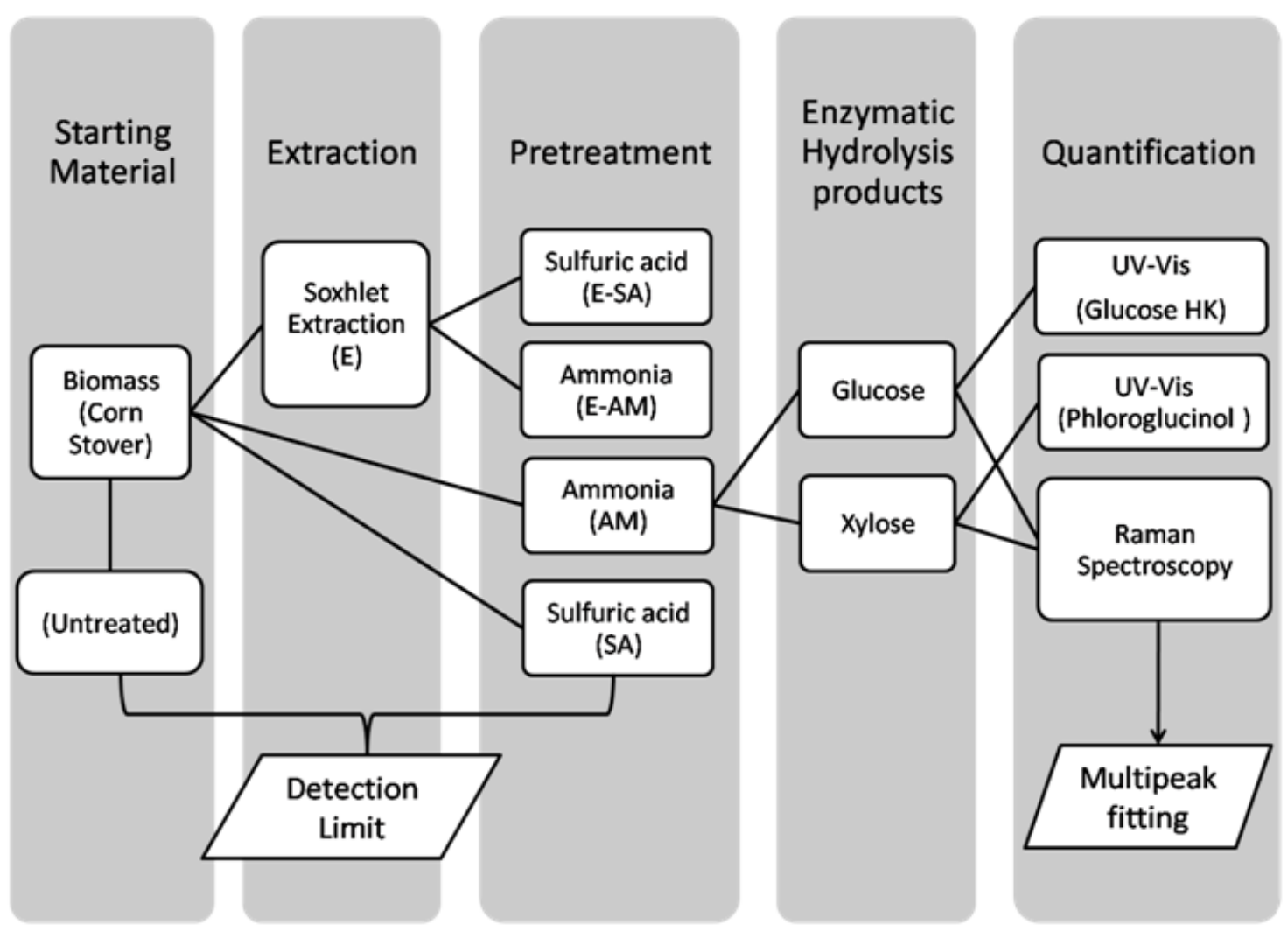


Figure 2

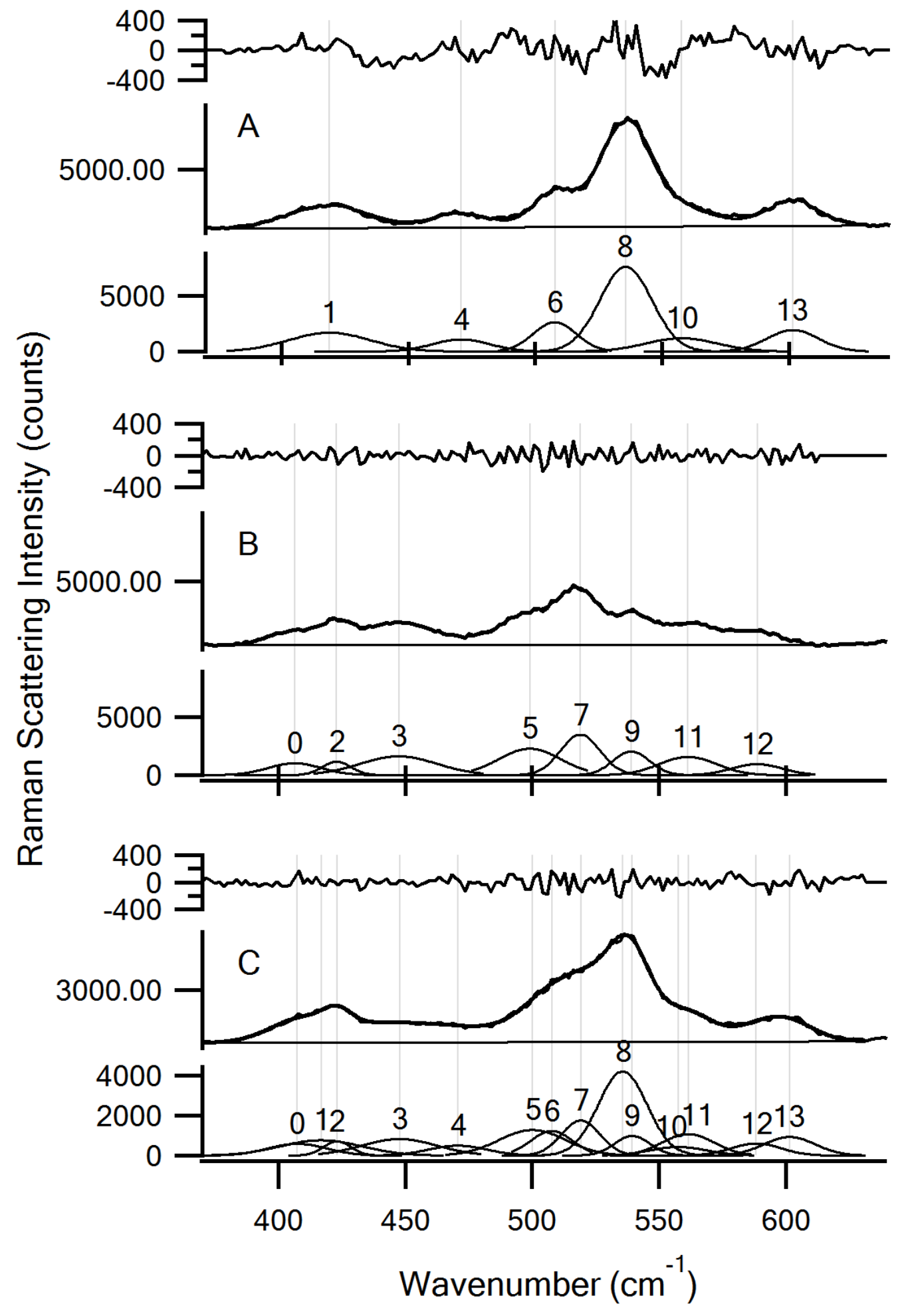




\section{CHAPTER 4: CONCLUSIONS}

This thesis focuses on developing quantitative spectroscopic methods to determine products from hydrolysis and fermentation reaction. A dispersive Raman spectrometer with $785 \mathrm{~nm}$ incident light is used to measure saccharides and ethanol in complex matrices. An appropriate pretreatment step is critical prior to hydrolysis reaction for reducing spectra interference. Different pretreatment methods are compared systematically and the glucose detection limits in hydrolysates are calculated.

Multipeak fitting model is also constructed in order to analyze two main sugars produced from cellulose and hemicellulose. Different ratios of sugar mixtures are used to examine the model's accuracy. Ammonia-pretreated corn stover is hydrolyzed by cellulase and hemicellulase, and concentrations of glucose and xylose in hydrolysate are determined by the multipeak fitting model.

The measurement is sensitive despite the complicated matrices from biomass conversion reactions. This protocol requires minimal sample preparation and analysis time with future automation capability. Raman spectroscopy is ideally suited for screening different biomass types, growing conditions or reaction conditions because it only takes seconds to minutes for each measurement and minimal spectral processing is required.

The developed multipeak fitting model enables accurate determination of glucose and xylose in hydrolysis broth. Similar methods could be applied to measure total sugar yields from other plant material with appropriate pretreatment. Raman spectroscopy has the potential to be used for real-time monitoring of reaction progress in hydrolysis broths that have complicated matrices. 


\title{
Appendix: Detection of cadmium(II) in water using colorimetric-solid phase extraction
}

\author{
A paper published in SAE International, Technical Paper 2005-01-28* \\ Chien-Ju Shih ${ }^{1}$, Neil C. Dias ${ }^{1}$, Marc D. Porter ${ }^{1,2}$
}

\begin{abstract}
Contamination of spacecraft water by heavy metals, such as cadmium and lead, is of growing concern. As a consequence, there is need for a rapid, on-board, easy-to-use method for the determination of cadmium(II) at low ppb levels in spacecraft drinking water supplies. This paper describes the preliminary development of a method for the selective, low level determination of cadmium(II) based on colorimetric-solid phase extraction (C-SPE) [1, 2]. In C-SPE, an analyte is extracted from a water sample onto a membrane that has been previously impregnated with a colorimetric reagent, and then quantified as its colorimetric complex directly on the membrane surface using diffuse reflectance spectroscopy. Results from preliminary tests that screened the performance of 1-(4-nitrophenyl)-3-(4-phenylazophenyl)triazene (cadion), and organic dyes, rhodamine B, brilliant green, and methyl violet as colorimetric reagents for potential use in a C-SPE analysis of cadmium(II) are described.
\end{abstract}

1.Ames Laboratory- U.S.D.O.E. and Department of Chemistry, Iowa State University, Ames, Iowa, 50011, USA.

*Reprint with permission from SAE International (Copy right @ 2005 SAE International) 


\section{INTRODUCTION}

Water samples collected on the International Space Station (ISS) have, on a few occasions, contained traces of lead(II), cadmium(II), and nickel(II)[3]. Currently, water quality analyses are performed using samples collected on ISS and returned to Earth. The time lapse between sample collection and analysis (up to 6 months), however, raises concerns about sample degradation during storage and transport. To alleviate this problem, fast, simple, sensitive methods to analyze water samples in microgravity environments need to be developed. Moreover, these methods must be selective, with minimal interferences from other components in the sample.

In-flight monitoring of heavy metals is vital to the maintenance of a safe supply of potable water. Cadmium, for instance, accumulates in the kidneys, liver, and lungs, potentially causing cancer and other dysfunctions[4]. Long-term effects of exposure to cadmium(II) also include bone embrittlement and blood disorders[5]. A number of methods have been approved for elemental analysis of water samples for cadmium by the U.S. Environmental Protection Agency, including graphite furnace atomic absorption spectroscopy[6], inductively coupled plasma emission spectroscopy[7], X-ray fluorescence spectroscopy[8], stripping voltammetry[9], radiochemical neutron activation analysis[10], and spectrophotometry with chromogenic reagents[11]. Spectrophotometry has proven particularly popular because of the low cost and ease-of-use. However, with the exception of various electrochemical formats, none of these methods have characteristics conducive to deployment in microgravity. 
Work in our laboratory, in conjunction with scientists from Wyle Laboratories at Johnson Space Center, has focused on colorimetric-solid phase extraction (C-SPE) as a method for the detection of a wide range of water quality indicators, including silver(I)[1], iodine and iodide[2], nickel(II)[12], and $\mathrm{pH}[13]$. The effectiveness of this methodology has also been demonstrated through KC-135 microgravity simulations[14]. C-SPE is based on the theory of solid phase extraction, which typically consists of three steps: 1) the analyte is concentrated by passing the liquid sample through an extraction membrane; 2) the analyte is desorbed from the membrane by a strongly eluting solvent; and 3) the eluent is analyzed by gas or liquid chromatography. C-SPE eliminates the elution step by quantifying the amount of an extracted, colorimetrically-labeled analyte directly on the membrane by using a diffuse reflectance spectrophotometer (DRS). C-SPE therefore reduces the total analysis time and eliminates the generation of organic waste while still maintaining the advantages of SPE (e.g., concentration factors of $\sim 1000$ ). In C-SPE, a syringe is filled with a known volume of sample, which is then usually metered through a membrane impregnated with a chromogenic reagent. The amount of colored complex extracted onto the membrane is then quantified using the measured reflectance and the Kubelka-Munk function, $F(R)$. The overall process typically requires only 1-2 min for completion, and often has a detection limit at ppb levels.

There are many colorimetric reagents that have been used for the determination of cadmium(II). Dyes like methylene blue (MB), methyl violet (MV), and rhodamine B react with cadmium halide complexes, which can be formed by the addition of KI to the aqueous solutions. Several azo dyes also form colored complexes with cadmium(II), such as 1-(2pyridylazo)-2-naphthol (PAN)[15], 4-(2-pyridylazo)-resorcinol (PAR)[16], 1-[(5-chloro-5pyridyl)azo]-2-naphthol[17], bromobenzo-thiazolyl-azocresol[18], 2,2'-(1,8-dihydroxy-3,6- 
isulfonaphthalene-2,7diazo) dibenzenearsonic acid (arsenazo III)[19], 1-[(6-bromo-2benzothiazolyl)azo]-2-naphthol (bromobenzothiazo, BBT)[20], and cadion[21, 22]. The most commonly used colorimetric reagent in the determination of cadmium is dithizone[23]. While sensitive, this method requires an extraction of the colored product with chloroform prior to spectrophotometric analysis, which precludes deployment on spacecraft. This paper describes the findings from a series of studies to screen for methodology that could potentially be adopted for an on-orbit analysis of Cd(II) by C-SPE.

\section{EXPERIMENTAL}

\section{REAGENTS AND CHEMICALS}

All aqueous solutions were prepared daily with deionized water that was further purified by a Millipore Milli-Q water purification system.

Dye Solutions - Rhodamine B, brilliant green, and methyl violet (Aldrich) aqueous solutions were prepared in deionized water to achieve $100-\mathrm{mL}$ solutions with a concentration of $3.0 \times 10^{-6} \mathrm{M}$.

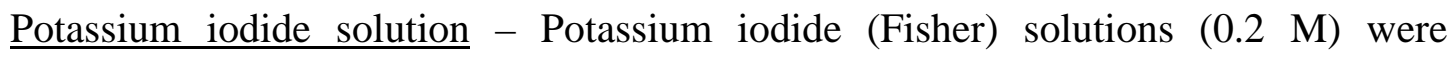
prepared in deionized water.

$\underline{\text { Standard cadmium(II) solutions }}$ - Cadmium chloride (>99.99\%, Aldrich) was dissolved in deionized water to prepare a cadmium stock solution (5.0 ppm). Lower concentrations were prepared by appropriate dilutions with deionized water.

$\underline{\mathrm{KOH}}$ aqueous solution - A 2.0 M potassium hydroxide (Fisher) solution was prepared by dissolving $2.8 \mathrm{~g}$ of potassium hydroxide in $25.0 \mathrm{~mL}$ of deionized water. 


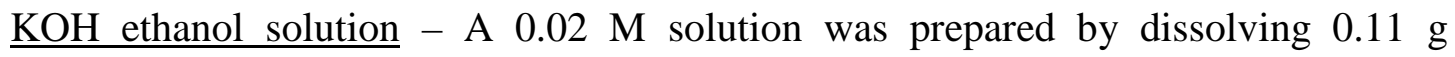
potassium hydroxide in ethanol and diluting to $100.0 \mathrm{~mL}$ with absolute ethanol (Fisher).

Cadion solution - A 0.02\% (w/v) 3-(4-nitrophenyl)-1-(4-phenylazophenyl)triazene (cadion, Aldrich) solution was prepared by dissolving $4 \mathrm{mg}$ of cadion in $20 \mathrm{~mL}$ of $0.02 \mathrm{M}$ KOH ethanol solution.

Potassium tartrate solution -Potassium tartrate (Fisher) was dissolved in deionized water to yield a $20 \% \mathrm{w} / \mathrm{w}$ solution.

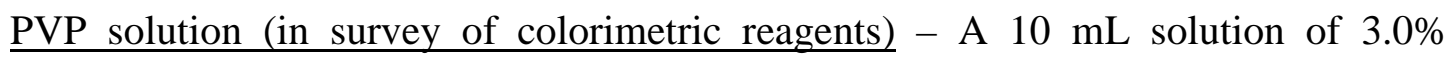
polyvinylpyrrolidone (PVP) (M.W.= 10,000, Aldrich) solution was prepared by dissolving $3.0 \mathrm{~g}$ of PVP in $100 \mathrm{~mL}$ 1:1(v/v) methanol: water.

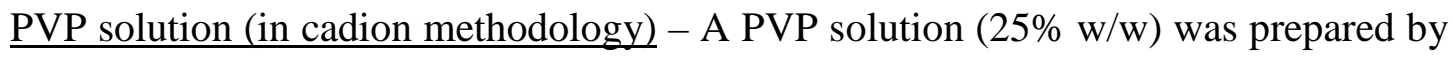

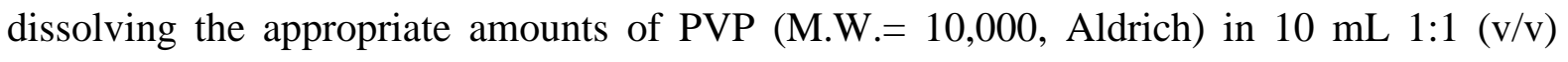
methanol: water.

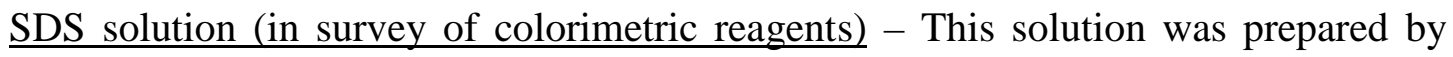
dissolving $7.0 \mathrm{~g}$ of sodium dodecyl sulfate (SDS, Aldrich) in a 100-mL volumetric flask with deionized water.

Cadion test solution - This solution was prepared by combining $1.0 \mathrm{~mL}$ of cadion solution, $2.5 \mathrm{~mL}$ of $\mathrm{KOH}$ aqueous solution, $5.0 \mathrm{~mL}$ of $95 \%$ ethanol aqueous solution, $0.5 \mathrm{~mL}$ potassium tartrate solution, $0.1 \mathrm{~mL}$ PVP (25\%, w/w) solution, and $10.0 \mathrm{~mL}$ deionized water in a 20 -mL glass vial.

$\underline{\text { C-SPE disks - Empore }}{ }^{\mathrm{TM}}$ SDB-XC $47 \mathrm{~mm}$ extraction membranes (3M) were used to collect the colorimetric complex. Membranes were cut into 13-mm diameter and placed in filter holders. 


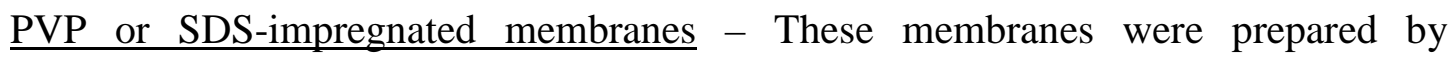
impregnating Empore SDB-XC 47-mm extraction membrane with PVP or SDS solution. Impregnation was accomplished by pulling $10 \mathrm{~mL}$ of 3\% PVP solution or the SDS solution through the membrane with a pressure difference of 200 torr. Residual solvent was removed by increasing the pressure difference to 500 torr for $1 \mathrm{~min}$. The membranes were then cut into 13-mm disks with a cork borer, and dried for 3-4 h at room temperature.

\section{INSTRUMENTATION}

A diffuse reflectance spectrophotometer (DRS) (Model LCB-6830, BYK-Gardner) was used to acquire spectral data from the disks. The DRS uses light emitting diodes to collect spectral data from 400-700 nm in 20-nm intervals. The battery-operated spectrophotometer is lightweight, small, and requires $\sim 2$ s to complete a single measurement. The diffusely reflected light is collected by an integrating sphere and directed to the detector. The spectrum is shown on the instrument display panel and can be downloaded to a personal computer.

\section{QUANTIFICATION}

The Kubelka-Munk function $(F(R))$ was used to determine the concentration of colored complex, $C$, as shown by the equation below[24]:

$F(R)=\frac{(1-R)^{2}}{2 R}=\frac{2.303 \varepsilon C}{s}$ 
where $R$ is the reflectance, $\varepsilon$ is the absorptivity of the colored complex, and $s$ is the scattering coefficient of the complex extracted on membrane surface. $F(R)$ is directly proportional to the concentration of the colorimetric complex if $\varepsilon$ and $s$ remain constant. The spectra were transferred to a computer by serial cable using BYK-Gardner QC-Link software and values of $F(R)$ at each wavelength were calculated from the resulting spreadsheet.

\section{RESULTS AND DISCUSSION}

\section{SURVEY OF COLORIMETRIC REAGENTS}

The first set of experiments compared the colorimetric reaction with cadmium(II) in water using various reagents: rhodamine B, brilliant green, and methyl violet. In each case, $10.0 \mathrm{~mL}$ of the cadmium(II) solution $(1.0 \mathrm{ppm}, \mathrm{pH} 3.5)$ was mixed with $1.0 \mathrm{~mL}$ of an aqueous solution of the dye and $1.0 \mathrm{~mL}$ of potassium iodide, forming a colored halide complex. This solution was then drawn into a plastic syringe and passed through a 13-mm membrane that was loaded in a filter holder previously and impregnated with PVP or SDS, noting that the impregnation of the membrane with PVP or SDS reflected attempts to increase the extraction efficiency of the membrane as prepared in earlier work[1]. After passing $60.0 \mathrm{~mL}$ air through the membrane, the extracted complex was analyzed by diffuse reflectance spectroscopy. Different analytical wavelengths were used (i.e., $560 \mathrm{~nm}$ for

rhodamine B, $640 \mathrm{~nm}$ for brilliant green, and $580 \mathrm{~nm}$ for methyl violet), reflecting the spectral properties of the complex. 
The results of these tests are shown in the Figure 1 and summarized in Table 1. These data have been corrected by subtracting the $F(R)$-value of the blank from that of the sample. The data indicate that rhodamine B yielded the greatest color change when complexed with Cd(II) and collected on an SDS-treated membrane. This result becomes more evident in Figure 1. In other words, the $F(R)$ of rhodamine B is clearly enhanced after reaction with anionic iodide complex ions $\left(\left[\mathrm{CdI}_{4}\right]^{2-}\right)$ whereas the other two dyes resulted in lower values of $F(R)$. These data suggest that cadmium(II)-rhodamine B complex binds more strongly to the membrane than unreacted rhodamine B because of the anionic exchange character of SDS.

Interferences with the determination caused by the presence of lead(II) and mercury(II), the most common cross-reactants in cadmium(II) determination were also examined. Each dye was mixed with separate solutions of the three different metal ions, each at a concentration of $1 \mathrm{ppm}$. Table 1 shows that all three dyes reacted to a notable and unacceptable extent with each metal ion.

\section{CADION METHODOLOGY}

A 5.0-mL plastic syringe was filled with $0.5 \mathrm{~mL}$ of cadion test solution and then 3.0 $\mathrm{mL}$ of a water sample; the complexation reaction was allowed to progress to completion ( 30 s). The complex was collected by passing the resulting solution through a 13-mm Empore SDB-XC extraction membrane, which was mounted in a swinnex filter holder. Next, an empty plastic syringe was used to pass $60 \mathrm{~mL}$ air through the membrane to remove residual liquid from the membrane surface. The filter holder was then opened and the extracted complex was measured directly on the membrane by the hand-held DRS. 
Cadion has been suggested as the most selective colorimetric reagent for cadmium(II) under carefully optimized conditions[22]. To test the possible adaptation of this reagent in CSPE, cadmium(II) standard solutions were prepared at concentrations of 5.0, 10.0, 50.0, and $200 \mathrm{ppb}$ by dilution of the stock solution with deionized water. In this experiment, $0.5 \mathrm{~mL}$ of the cadion test solution were reacted with $3.0 \mathrm{~mL}$ of the cadmium(II) solution by mixing in the syringe for 1-2 min, followed by extraction of the colored complex on the Empore disks. This reaction is expected to form a 1:3 analyte:chelate complex, which requires excess of cadion. We add that the cadion test solution contains potassium tartrate as a masking agent and PVP as a dispersing agent. After passing $60.0 \mathrm{~mL}$ air through the membrane, the reflectance of the colored complex and excess cadion on the membrane was measured by the diffuse reflectance spectroscopy.

The resulting spectra are shown in Figure 2. As is evident, the $F(R)$ value at $580 \mathrm{~nm}$ decreased when the concentration of cadmium(II) in solution increased. This decrease represents the depletion of cadion due to its complexation with cadmium. Using $580 \mathrm{~nm}$ as the analytical wavelength, which corresponds to the absorption maximum for unreacted cadion, the calibration curve in Figure 3 was generated. This plot exhibits a linear correlation between $F(R)$ and cadmium(II) over a concentration range of 0 to $200 \mathrm{ppb}$, spanning the range mandated by NASA regarding water quality monitoring requirements (1-100 ppb). It is also important to note that unreacted cadion when extracted on the membrane appears to undergo a gradual color change after about 3 min, which may reflect the dehydration of the complex. Tests to immobilize cadion test solution and investigate cross-reactivity of cadion and foreign ions are underway. 


\section{CONCLUSION}

This paper provides an update on the development of cadmium(II) detection using C-SPE. Of the screened methods, the cadion method appears to be the most promising. It has the potential to be rapid, sensitive and selective, thereby fulfilling several of the performance requirements for ISS and spacecraft water quality monitoring. Many parameters have yet to be optimized in this experiment, and we are pursuing issues related to interferences and reproducibility with the goal of testing the platform under microgravity conditions in the near future.

\section{ACKNOWLEDGMENTS}

This work was supported by NASA contract NAG9-1510. The Ames Laboratory is operated by Iowa State University under US department of Energy contract W7405-eng-82.

\section{REFERENCES}

(1)Arena, M. P.; Porter, M. D.; Fritz, J. S. Anal. Chim Acta 2003, 482, 197-207.

(2)Arena, M. P.; Porter, M. D.; Fritz, J. S. Anal. Chem. 2002, 74, 185-190.

(3)Plumlee, D. K., et al. 32nd International Conference on Environmental Systems, San Antonio, TX 2002, SAE Technical Paper \#2002-01-2537.

(4)Robards, K.; Worsfold, P. Analyst (Cambridge, United Kingdom) 1991, 116, 549-568.

(5)Alfven, T.; Jaerup, L.; Elinder, C.-G. Environmental Health Perspectives 2002, 110, A 505. 
(6)Tsalev, D. L. Atomic Absorption Spectrometry in Occupational and Environmental Health Practice, Vol. 2: Determination of Individual Elements, 1984.

(7) Stoeppler, M. In Biological Monitoring of Toxic Metals; Clarkson, T. W., Friberg, L., Nordberg, G. F., Sager, P. R., Eds.; Plenum Press: New York, 1988, pp 481-497.

(8) Skoog, D. A. Principles of Instrumental Analysis. 3rd Ed, 1985.

(9) Bard, A. J.; Faulkner, L. R. Electrochemical Methods: Fundamentals and Applications, 1982.

(10) Bedregal, P. S.; Montoya, E. H. J. of Radioanal. and Nu. Chem. 2002, 254, 363-364.

(11) Marczenko, Z. Separation and Spectrophotometric Determination of Elements, 1986.

(12)Gazda, D. B.; Fritz, J. S.; Porter, M. D. Anal. Chim Acta 2004, 508, 53-59.

(13) Gazda, D. B.; Fritz, J. S.; Porter, M. D. Anal. Chem. 2004, 76, 4881-4887.

(14) Colorimetric Solid Phase Extraction Measurements of Spacecraft Dringking Water Contaminants 2004, KC-135 Postflight Report.

(15) Berger, W.; Elvers, H. Z. Anal. Chem 1964, 199, 166.

(16) Kitano, M.; Ueda, J. J. Chem. Soc. Japan, Pure Chem. Sect. 1970, 91, 760.

(17) Shibata, S.; Furukawa, M.; Ishiguro, Y. Mikrochimica Acta 1972, 721-727.

(18) Gusev, S. I.; Zhvakina, M. V.; A., K. I. Zh. Analit. Khim. 1971, 26, 1493.

(19) Michaylova, V.; Yoroukova, L. Anal. Chim. Acta 1974, 68, 73.

(20) Shkrobot, E. P.; Bakinovskaya, L. M. Zavodsk. Lab. 1966, 32, 1452.

(21) Chavanne, P.; Geronimi, C. Anal. Chim. Acta 1958, 19, 377-388.

(22) Watanabe, H.; Ohmori, H. Talanta 1979, 26, 959-961.

(23) Thiagarajau, N.; Subajau, M. Anal. Chim. Acta 1992, 269, 269. 
(24) Kortum, G. In Reflectance Spectroscopy-Principles, Methods, Applications; Springer: New York, 1969, pp 106-116.

\section{CONTACT}

Correspondence regarding this article should be addressed to Dr. Marc D. Porter of Iowa State University, Ames, IA 50011. E-mail address: mporter@porter1.ameslab.gov 
Table 1. $F(R)$ of cadmium complexed with organic dyes and $\mathrm{Cd}(\mathrm{II}), \mathrm{Pb}(\mathrm{II})$, and $\mathrm{Hg}(\mathrm{II})$.

\begin{tabular}{|c|c|c|c|}
\hline \multirow{2}{*}{ Dye } & Metal & \multicolumn{2}{l}{ Extraction condition } \\
\cline { 3 - 4 } & ion & KI/PVP & KI/SDS \\
\hline \multirow{3}{*}{ Rhodamine B } & $\mathrm{Cd}$ & -0.023 & 0.245 \\
\cline { 2 - 4 } & $\mathrm{Pb}$ & -0.296 & 0.200 \\
\cline { 2 - 4 } & $\mathrm{Hg}$ & -0.114 & 0.211 \\
\hline \multirow{3}{*}{ Brilliant Green } & $\mathrm{Cd}$ & 0.115 & -0.709 \\
\cline { 2 - 4 } & $\mathrm{Pb}$ & 0.167 & -0.716 \\
\cline { 2 - 4 } & $\mathrm{Hg}$ & 0.123 & -1.073 \\
\hline \multirow{3}{*}{ Methyl Violet } & $\mathrm{Cd}$ & 0.092 & -0.146 \\
\cline { 2 - 4 } & $\mathrm{Pb}$ & 0.047 & -0.164 \\
\cline { 2 - 4 } & $\mathrm{Hg}$ & 0.137 & -0.267 \\
\hline
\end{tabular}



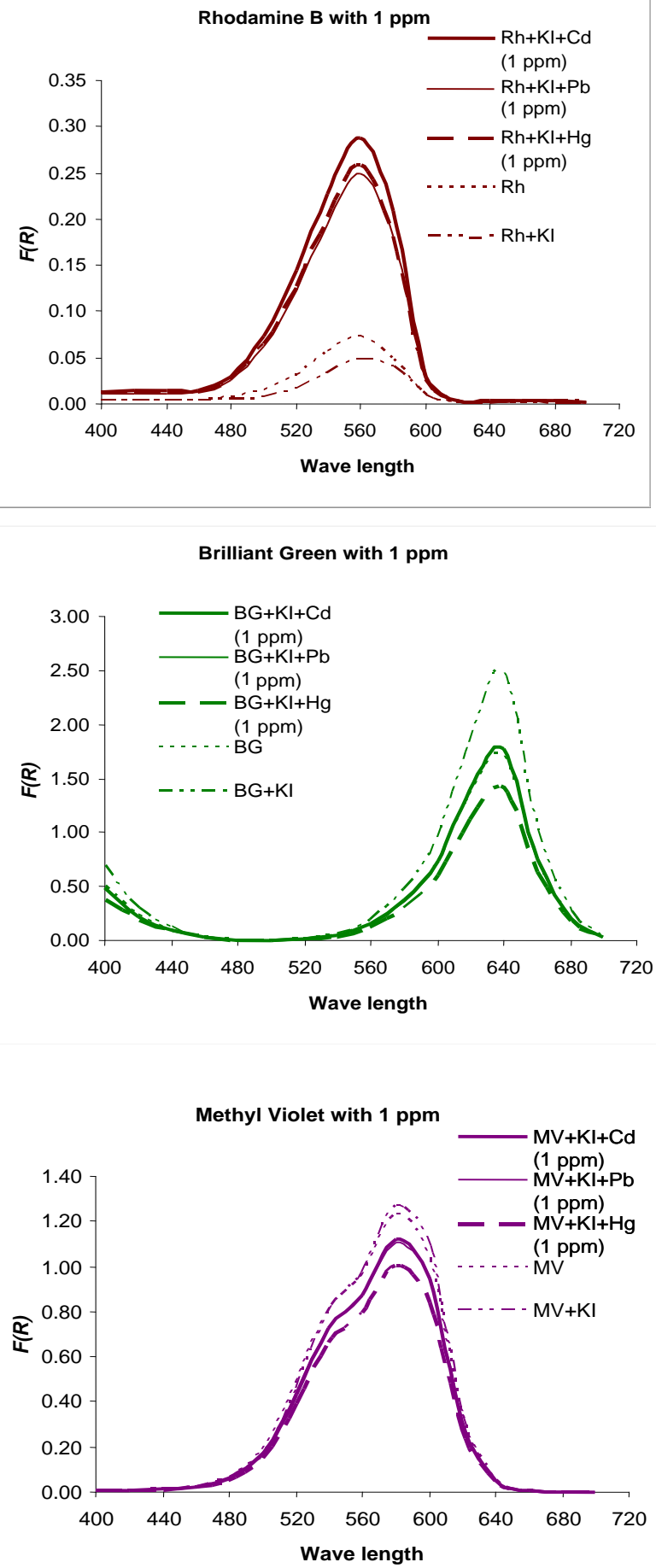

Figure 1. Spectra of cadimiotetraiodide-dye complex on SDS-treated membrane, from top to the bottom: rhodamine $\mathrm{B}$, brilliant green and methyl violet. 


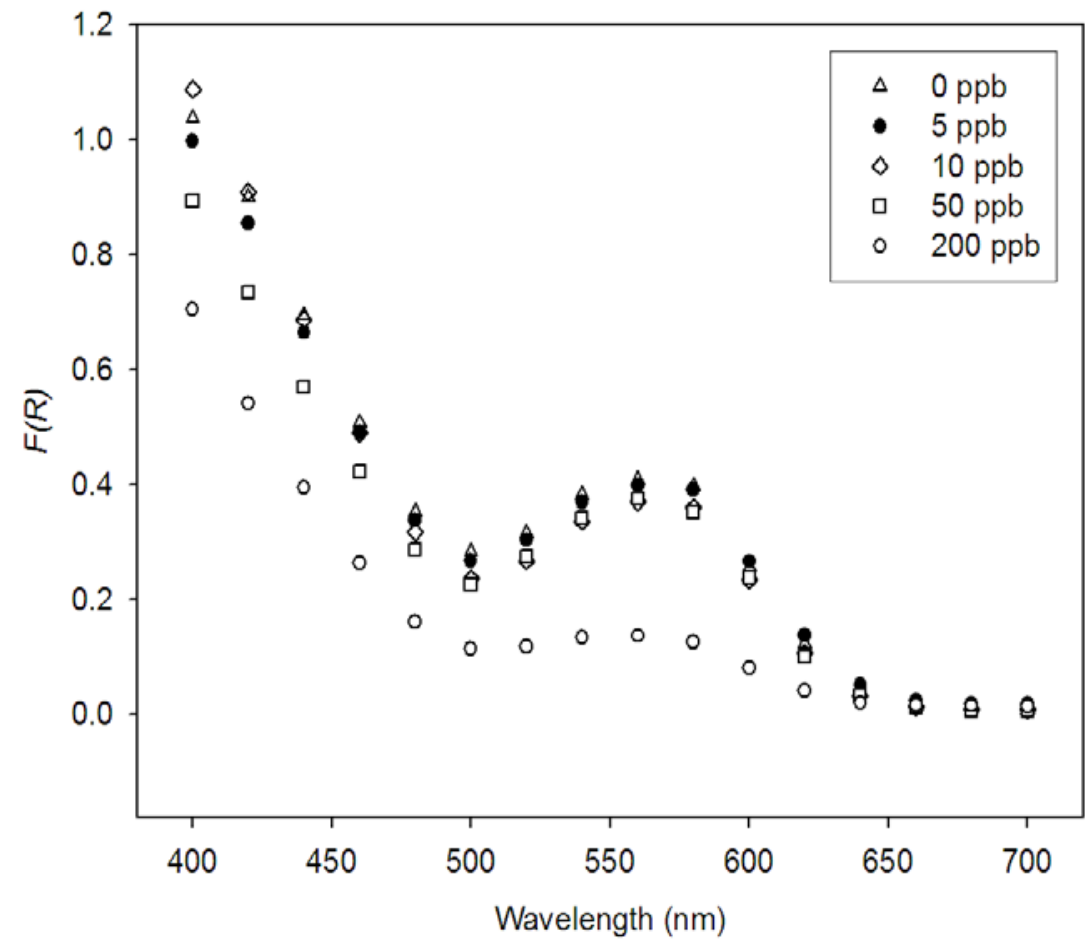

Figure 2. Spectra of cadmium(II)-cadion complex with excess of cadion in different cadmium(II) concentrations. 


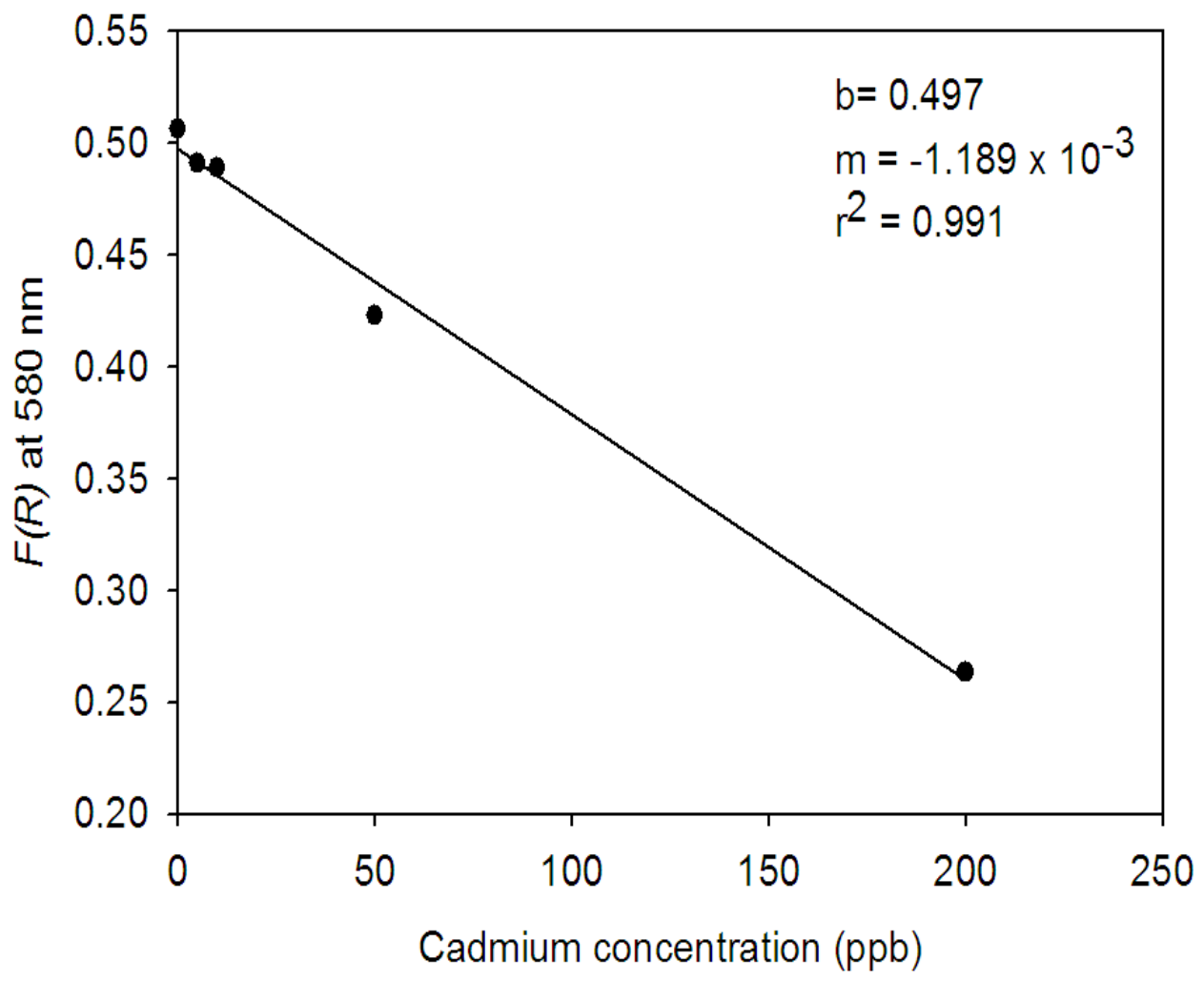

Figure 3. Calibration plot of $F(R)$ at $580 \mathrm{~nm}$ as a function of cadmium(II) concentration. 